\title{
TeV-scale vector leptoquark from Pati-Salam unification with vectorlike families
}

\author{
Syuhei Iguro, ${ }^{1}$ Junichiro Kawamura $\odot,{ }^{2,3}$ Shohei Okawa, ${ }^{4}$ and Yuji Omura ${ }^{5}$ \\ ${ }^{1}$ Department of Physics, Nagoya University, Nagoya 464-8602, Japan \\ ${ }^{2}$ Center for Theoretical Physics of the Universe, Institute for Basic Science (IBS), Daejeon 34051, Korea \\ ${ }^{3}$ Department of Physics, Keio University, Yokohama 223-8522, Japan \\ ${ }^{4}$ Department of Physics and Astronomy, University of Victoria, Victoria, \\ British Columbia V8P 5C2, Canada \\ ${ }^{5}$ Department of Physics, Kindai University, Higashi-Osaka, Osaka 577-8502, Japan
}

(Received 13 April 2021; accepted 26 August 2021; published 4 October 2021)

\begin{abstract}
In this paper, we show an explicit way to realize a TeV-scale vector leptoquark from the Pati-Salam unification with extra vectorlike families. The leptoquark mass is constrained to be heavier than PeV scale by the measurement of a flavor violating kaon decay, $K_{L} \rightarrow \mu e$, in conventional models. This strong constraint can be avoided by introducing vectorlike families consistently with the quark and lepton masses and Cabbibo-Kobayashi-Maskawa and Pontecorvo-Maki-Nakagawa-Sakata matrices. In this model, the vector leptoquark can be sufficiently light to explain the recent $b \rightarrow s \mu \mu$ anomaly, while the $b \rightarrow c \tau \nu$ anomaly is difficult to be explained due to the strong constraints from the $Z^{\prime}$ boson and vectorlike quark searches at the LHC. When the $b \rightarrow s \mu \mu$ anomaly is explained, we show that $\mathcal{O}(0.2) \%$ tuning is required in the fermion matrix, the future experiments in $\mu \rightarrow e \gamma$ and $\mu$-e conversions will cover most available parameter space, and sizable neutral meson mixings, induced by the extra Higgs doublets, are unavoidable.
\end{abstract}

DOI: 10.1103/PhysRevD.104.075008

\section{INTRODUCTION}

A leptoquark is a hypothetical boson that carries both the baryon number and lepton number [1]. Recently, the phenomenology of $\mathrm{TeV}$-scale leptoquarks has been discussed in connection with tensions between experimental results and theoretical explanations in semileptonic $B$ meson decays [2-16]. An attractive extension that predicts leptoquarks is the Pati-Salam (PS) unification [17]. In this paper, we explore a possibility of incorporating a vector leptoquark at $\mathrm{TeV}$ scale in the PS model, consistently with the observed standard model (SM) fermion masses and various experimental results.

In the minimal setup of the PS unification, quarks and leptons are unified into two chiral multiplets under the PS gauge symmetry, $G_{\mathrm{PS}}=S U(4)_{C} \times S U(2)_{L} \times S U(2)_{R}$. The hypercharge is quantized since all gauge symmetries are non-Abelian. The $S U(2)_{R}$ symmetry, a right-handed analog of the weak isospin symmetry $S U(2)_{L}$, requires the introduction of right-handed neutrinos, which allows for neutrino mass generation in a number of ways. A vector

Published by the American Physical Society under the terms of the Creative Commons Attribution 4.0 International license. Further distribution of this work must maintain attribution to the author(s) and the published article's title, journal citation, and DOI. Funded by SCOAP ${ }^{3}$. leptoquark arises as the massive degree of freedom associated with the $G_{\mathrm{PS}}$ breaking, $S U(4)_{C} \rightarrow S U(3)_{C} \times$ $U(1)_{B-L}$, and hence its mass will be around the PS breaking scale.

While the PS symmetry is normally considered to be broken at high scales, the breaking scale can be lowered to scales a few orders of magnitude above the electroweak (EW) scale due to the absence of bosons with di-quark couplings, which induce the proton decay. However, lowering the PS breaking scale, or equally the leptoquark mass, causes some difficulties. First, the PS symmetry predicts Yukawa unification for the SM fermions, providing mass equality relations between quarks and leptons, $m_{d}=m_{e}$ and $m_{u}=m_{\nu}^{\text {dirac }}$. These relations are obviously inconsistent with observation at low energy. In a conventional scenario [18], the observed mass spectrum is achieved by taking renormalization group (RG) effects and corrections from higher-dimensional operators into account. This scenario, however, requires that the $G_{\mathrm{PS}}$ breaking scale is around the conventional grand unified theory (GUT) scale, $\mathcal{O}\left(10^{16} \mathrm{GeV}\right)$, in order to obtain sufficiently large corrections from the RG effects and higher dimensional operators, the latter of which are usually suppressed by the Planck scale. The second difficulty is brought by rare meson decays. The vector leptoquark from the PS breaking mediates a variety of flavor violating transitions such as $K_{L} \rightarrow \mu e, B_{d} \rightarrow \tau e$, etc. The most stringent limit is set by 
the $K_{L} \rightarrow \mu e$ decay and its measurement restricts the leptoquark mass to be heavier than $\mathrm{PeV}[19,20]$, although this restriction can be relaxed down to $\sim 100 \mathrm{TeV}$ if the tau lepton is associated with the first generation quarks [20]. Therefore, the low-scale realization of the PS symmetry breaking requires modifications to the conventional PS model.

An attempt to achieve the low-scale PS breaking is made, for example, in Ref. [21], where a couple of vectorlike copies of fundamental chiral multiplets are added to the minimal setup. In such a model, SM quarks and leptons originate in different PS multiplets which are distinguished by an additionally imposed global symmetry, which helps to disentangle the strong mass equality relations and also to suppress the rare meson decays event with the TeV-scale PS breaking. Similar attempts in models with exotic representation fermions are recently studied in Ref. [22]. Other variants of the PS model, which overcome in different ways the above-mentioned problems in realizing $\mathrm{TeV}$-scale vector leptoquarks, include Refs. [12,16,23-29].

Many of these attempts to lower the PS scale are strongly motivated by the recent experimental anomalies in the semileptonic $B$ meson decays, one of the recent hot topics in high energy physics. A number of experimental results suggest deviations from the SM predictions in the measurements of $B \rightarrow K^{(*)} \ell \ell(\ell=e, \mu)$ and $B \rightarrow D^{(*)} \tau \nu$ processes. In regard to the former process, the $\mathrm{LHCb}$ collaboration reports a measurement of the ratio of the branching ratio of $B \rightarrow K^{(*)} e e$ to that of $B \rightarrow K^{(*)} \mu \mu$. The result tells that the branching ratio of $B \rightarrow K^{(*)} \mu \mu$ is slightly smaller than the SM prediction [30-33]. The LHCb also investigates the observables related to the angular distribution of the $B \rightarrow K^{*} \mu \mu$ decay, and shows that one observable, namely $P_{5}^{\prime}$, deviates from the SM prediction $[34,35]$. Both deviations can be consistently explained by the new physics contribution to the $b \rightarrow s \mu \mu$ transition at the parton level. The recent observation for the angular observables in charged $B$ meson decay also supports the result [36]. We call the discrepancies of this kind the $b \rightarrow$ $s \mu \mu$ anomaly. In addition, another discrepancy is found in $B \rightarrow D^{(*)} \tau \nu$. The decay mode has been studied in the $B A B A R$, the Belle and the LHCb experiments. The BABAR collaboration has announced that the experimental result on the lepton universality, where the branching ratio of $B \rightarrow$ $D^{(*)} \tau \nu$ is compared with $B \rightarrow D^{(*)} \ell \nu$, is largely deviated from the SM prediction $[37,38]$. We call this discrepancy the $b \rightarrow c \tau \nu$ anomaly. The Belle [39-42] and the LHCb $[43,44]$ have also measured the same quantity and have reported their results; the discrepancy becomes milder but the world average is still about $3-4 \sigma$ away from the SM value [45-50].

The announcement of those anomalies has triggered a variety of new physics studies, ranging from purely effective field theory analyses to the construction of UV complete descriptions of the SM. Among many proposed possibilities, new physics models containing a vector leptoquark $U_{1}$ with the quantum number $(\mathbf{3}, \mathbf{1}, 2 / 3)$ under the SM gauge group, $G_{\mathrm{SM}}=S U(3)_{C} \times S U(2)_{L} \times U(1)_{Y}$, are particularly attractive. Such models can provide a coherent explanation for both $b \rightarrow s \mu \mu$ and $b \rightarrow c \tau \nu$ anomalies. One well-motivated class of such models is the PS model. Indeed, it is shown that some variants of the PS model with the $U_{1}$ leptoquark can accommodate both sets of the anomalies [12,16,23-27]. Other theoretical activities to address the anomalies in models with $U_{1}$ or other representation leptoquarks are found in Refs. [4-7, $9,28,29,47,51-55]{ }^{1}$

In this paper, we construct a simple model based on the PS gauge theory that realizes a $\mathrm{TeV}$-scale vector leptoquark and accounts for the observed SM fermion masses and mixings as well as the measurements of the flavor violating processes. We then study the low-energy phenomenology of the model. To this end, we introduce vectorlike fermions to the minimal PS model. The traditional realization of the $\mathrm{TeV}$ leptoquark is faced with the measurement of $K_{L} \rightarrow \mu e$, while mixings between the chiral and vectorlike fermions relax the constraint in our model. A similar attempt is already made in Ref. [21], but our construction is different in the following points. First, we try to build a setup as economically as possible, so we do not introduce many additional scalar fields that are required in Ref. [21] to generate the fermion mass splittings because of an extra global symmetry. We expect that the model proposed in this paper is the minimal extension to realize the observed fermion masses and avoid the experimental constraints. Second, we carefully look at flavor physics inevitably induced by the leptoquark and extra scalars such as PS scalars and EW Higgs bosons. We also discuss if the recent $B$ physics anomalies can be accommodated in this kind of model. As a result, it turns out that lepton flavor violating processes are enhanced in the presence of the heavy vectorlike fermions and hence are particularly important to test the model. We also point out that parameters of the model should be tuned at $\mathcal{O}(0.1 \%)$ level to be consistent with the realistic fermion masses and mixing as well as flavor constraints from $K_{L} \rightarrow \mu e$ etc., if the $b \rightarrow s \mu \mu$ anomaly is mediated by the leptoquark. Interestingly, most of the parameter space will be covered by future experiments searching for the $\mu-e$ conversion process and $\mu \rightarrow e \gamma$. We also show that the $b \rightarrow c \tau \nu$ anomaly can be hardly explained in this model due to the constraints from the $Z^{\prime}$ boson search at the LHC.

\footnotetext{
${ }^{1}$ If one gives up explaining both sets of the anomalies, other new physics models are possible. The $b \rightarrow s \mu \mu$ anomaly is explained by a $Z^{\prime}$ boson at the tree level [56-62] or one-loop box diagrams mediated by extra fermions [63-72]. The explanation of the $b \rightarrow c \tau \nu$ anomaly with a charged Higgs boson is extensively discussed [73-82], while such possibilities are constrained by the collider search[83] and $B_{c} \rightarrow \tau \nu$ decay [7,84-86].
} 
This paper is organized as follows. In Sec. II, we introduce our PS model and suggest a structure of fermion mass matrices that helps to suppress the rare meson decays. In Sec. III, we discuss the phenomenology of the constructed model with a particular focus on flavor physics and the $b \rightarrow s \mu \mu$ anomaly. Section IV is devoted to a summary. The details of the model and numerical analysis are shown in Appendixes A and B, respectively.

\section{PATI-SALAM MODEL WITH VECTORLIKE FERMIONS}

We shall consider a model with the PS gauge symmetry, $G_{\mathrm{PS}}=S U(4)_{C} \times S U(2)_{L} \times S U(2)_{R}$. In the minimal setup with $G_{\mathrm{PS}}$, there are three generations of chiral fermions $L$, $R$ and a bi-doublet Higgs field $\Phi$. The Yukawa couplings of the chiral fermions are given by

$$
-\mathcal{L}_{Y}^{\mathrm{Ch}}=\bar{L} y_{1} \Phi R+\bar{L} y_{2} \epsilon^{T} \Phi^{*} \epsilon R+\text { H.c., }
$$

where $\epsilon:=i \sigma_{2}$ acts onto the $S U(2)_{L}$ and $S U(2)_{R}$ indices. Here, $y_{1}$ and $y_{2}$ are the $3 \times 3$ Yukawa matrices in the flavor space. The linear combination of the two terms leads the splittings of the Yukawa couplings between the up-type and down-type quarks, as well as the charged leptons and neutrinos. However, since the $\Phi$ does not carry the $S U(4)_{C}$ charge, the down-type quarks and charged leptons which form the $S U(4)_{C}$ multiplet have the same Yukawa couplings at the tree level, resulting in no mass splitting between them. It is often considered that the mass differences are generated by RG effects after the PS gauge symmetry breaking and/or by incorporating higher-dimensional operators involving $S U(4)_{C}$ symmetry breaking vacuum expectation values (VEVs). The symmetry breaking scale may need to be around the conventional GUT scale $\sim 10^{16} \mathrm{GeV}$, in order to realize sufficiently large corrections from the RG effects and/or higher-dimensional operators. Thus, the realistic fermion masses will require modifications to the minimal setup [Eq. (2.1)] if the $G_{\mathrm{PS}}$ breaking scale is around TeV.

In this paper, we extend the minimal setup to realize the PS breaking at $\mathrm{TeV}$ scale, consistently with the observed SM fermion masses and mixings as well as the measurements of the flavor violating processes. The matter content and the charge assignment in our extension are summarized in Table I. The PS gauge symmetry is expected to be spontaneously broken at the $\mathrm{TeV}$ scale in our setup. We introduce extra vectorlike fermions, an $S U(4)_{C}$ adjoint scalar $\Delta$, and another scalar $\Sigma,(\overline{\mathbf{1 0}}, \mathbf{1}, \mathbf{3})$. The mass splittings between the charged leptons and down quarks are generated by the VEV of $\Delta$. The VEV of $\Sigma$ induces the Majorana masses for the right-handed neutrinos. In this model, the nonzero VEV of $\Delta$ breaks $S U(4)_{C}$ to $S U(3)_{C} \times$ $U(1)_{B-L}$ and that of $\Sigma$ breaks $S U(2)_{R} \times U(1)_{B-L}$ to $U(1)_{Y}$.

The vectorlike fermions are denoted by $F_{L}, F_{R}, f_{L}$ and $f_{R}$. Each type of vectorlike fermion has three flavors as
TABLE I. The matter content in the Pati-Salam model. Each type of fermion has three flavors.

\begin{tabular}{lcccc}
\hline \hline Fields & Spin & $S U(4)_{C}$ & $S U(2)_{L}$ & $S U(2)_{R}$ \\
\hline$L$ & $1 / 2$ & $\mathbf{4}$ & $\mathbf{2}$ & $\mathbf{1}$ \\
$R$ & $1 / 2$ & $\mathbf{4}$ & $\mathbf{1}$ & $\mathbf{2}$ \\
$F_{L}$ & $1 / 2$ & $\mathbf{4}$ & $\mathbf{2}$ & $\mathbf{1}$ \\
$F_{R}$ & $1 / 2$ & $\mathbf{4}$ & $\mathbf{2}$ & $\mathbf{1}$ \\
$f_{L}$ & $1 / 2$ & $\mathbf{4}$ & $\mathbf{1}$ & $\mathbf{2}$ \\
$f_{R}$ & $1 / 2$ & $\mathbf{4}$ & $\mathbf{1}$ & $\mathbf{2}$ \\
$\Delta$ & 0 & $\mathbf{1 5}$ & $\mathbf{1}$ & $\mathbf{1}$ \\
$\Sigma$ & 0 & $\overline{\mathbf{1 0}}$ & $\mathbf{1}$ & $\mathbf{3}$ \\
$\Phi$ & 0 & $\mathbf{1}$ & $\mathbf{2}$ & $\overline{\mathbf{2}}$ \\
\hline \hline
\end{tabular}

the chiral fermions. As shown in Table I, the charge assignment of $L(R)$ is the same as that of $F_{L}\left(f_{R}\right)$. In our work, we simply assume that there is an underlying theory or some symmetry, and they can be distinguished from each other. This is the minimal setup such that the required fermion mass splittings are generated and dangerous flavor violating processes are suppressed, which will be shown later. The vectorlike mass terms and Yukawa couplings are given by

$$
\begin{aligned}
- & \mathcal{L}_{Y}^{\mathrm{VL}}=\bar{F}_{L} M_{L} F_{R}+\bar{f}_{L} M_{R} f_{R}+\bar{f}_{L} m_{R} R+\bar{L} m_{L} F_{R} \\
& +\bar{F}_{L} \kappa_{L} \Delta F_{R}+\bar{f}_{L} \kappa_{R} \Delta f_{R}+\bar{f}_{L} \epsilon_{R} \Delta R+\bar{L} \epsilon_{L} \Delta F_{R} \\
& +\bar{F}_{L} \lambda_{1} \Phi R+\bar{F}_{L} \lambda_{2} \epsilon^{T} \Phi^{*} \epsilon R+\bar{L}_{1} \Phi f_{R}+\bar{L} \tilde{\lambda}_{2} \epsilon^{T} \Phi^{*} \epsilon f_{R} \\
& +\bar{F}_{L} \tilde{y}_{1} \Phi f_{R}+\bar{F}_{L} \tilde{y}_{2} \epsilon^{T} \Phi^{*} \epsilon f_{R}+\bar{f}_{L} \tilde{y}_{1}^{\prime} \Phi^{\dagger} F_{R} \\
& +\bar{f}_{L} \tilde{y}_{2}^{\prime} \epsilon^{T} \Phi^{T} \epsilon F_{R}+\text { H.c. }
\end{aligned}
$$

where all the masses and Yukawa couplings are $3 \times 3$ matrices in the flavor space. Here, the first line is the treelevel vectorlike mass terms. The second line is the Yukawa couplings with the $S U(4)_{C}$ adjoint $\Delta$ and the last two lines are the Yukawa couplings with the bi-doublet $\Phi$. The mass splittings between quarks and leptons are induced by the Yukawa couplings involving the adjoint scalar $\Delta$. In the next subsection, we study the fermion mass matrices originated from the spontaneous PS symmetry breaking.

In addition to Eq. (2.2), there are Yukawa couplings involving $\Sigma$, which induce Majorana masses after the symmetry breaking:

$$
-\mathcal{L}_{\Sigma}^{y}=\frac{1}{2} \bar{f}_{R}^{c} h \epsilon^{T} \Sigma f_{R}+\text { H.c., }
$$

where $h$ is the $3 \times 3$ symmetric Yukawa matrix. Here, we simply assumed that the Yukawa couplings involving $R$ are vanishing and the effective Majorana mass terms consist of only $f_{R}{ }^{2}$

\footnotetext{
${ }^{2}$ We note that this situation can be realized by assigning extra symmetry to distinguish $f_{R}$ from $R$.
} 


\section{A. Fermion masses}

After the $G_{\mathrm{PS}}$ breaking, the fermion multiplets are decomposed as

$$
\begin{aligned}
& L=\left(\begin{array}{cc}
e_{L} & n_{L} \\
d_{L} & u_{L}
\end{array}\right), \quad F_{L}=\left(\begin{array}{cc}
E_{L} & N_{L} \\
D_{L} & U_{L}
\end{array}\right), \quad f_{L}=\left(\begin{array}{cc}
\mathcal{E}_{L} & \mathcal{N}_{L} \\
\mathcal{D}_{L} & \mathcal{U}_{L}
\end{array}\right), \\
& R=\left(\begin{array}{cc}
e_{R} & n_{R} \\
d_{R} & u_{R}
\end{array}\right), \quad F_{R}=\left(\begin{array}{cc}
E_{R} & N_{R} \\
D_{R} & U_{R}
\end{array}\right), \quad f_{R}=\left(\begin{array}{cc}
\mathcal{E}_{R} & \mathcal{N}_{R} \\
\mathcal{D}_{R} & \mathcal{U}_{R}
\end{array}\right),
\end{aligned}
$$

where the rows are the $S U(4)_{C}$ space and the columns are the $S U(2)_{L(R)}$ space for the $S U(2)_{L(R)}$ doublets. We parametrize the Dirac mass matrices as

$$
\begin{aligned}
&-\mathcal{L}_{Y}^{\mathrm{Ch}}-\mathcal{L}_{Y}^{\mathrm{VL}}=\left(\begin{array}{c}
\bar{u}_{L} \\
\bar{U}_{L} \\
\overline{\mathcal{U}}_{L}
\end{array}\right)^{T}\left(\begin{array}{ccc}
y_{u} v_{H} & \tilde{\lambda}_{u} v_{H} & m_{Q_{L}} \\
\lambda_{u} v_{H} & \tilde{y}_{u} v_{H} & M_{Q_{L}} \\
m_{Q_{R}} & M_{Q_{R}} & \tilde{y}_{u}^{\prime} v_{H}
\end{array}\right)\left(\begin{array}{c}
u_{R} \\
\mathcal{U}_{R} \\
U_{R}
\end{array}\right)+\left(\begin{array}{c}
\bar{d}_{L} \\
\bar{D}_{L} \\
\overline{\mathcal{D}}_{L}
\end{array}\right)^{T}\left(\begin{array}{ccc}
y_{d} v_{H} & \tilde{\lambda}_{d} v_{H} & m_{Q_{L}} \\
\lambda_{d} v_{H} & \tilde{y}_{d} v_{H} & M_{Q_{L}} \\
m_{Q_{R}} & M_{Q_{R}} & \tilde{y}_{d}^{\prime} v_{H}
\end{array}\right)\left(\begin{array}{c}
d_{R} \\
\mathcal{D}_{R} \\
D_{R}
\end{array}\right) \\
&+\left(\begin{array}{c}
\bar{e}_{L} \\
\bar{E}_{L} \\
\overline{\mathcal{E}}_{L}
\end{array}\right)^{T}\left(\begin{array}{ccc}
y_{d} v_{H} & \tilde{\lambda}_{d} v_{H} & m_{\ell_{L}} \\
\lambda_{d} v_{H} & \tilde{y}_{d} v_{H} & M_{\ell_{L}} \\
m_{\ell_{R}} & M_{\ell_{R}} & \tilde{y}_{d}^{\prime} v_{H}
\end{array}\right)\left(\begin{array}{c}
e_{R} \\
\mathcal{E}_{R} \\
E_{R}
\end{array}\right)+\left(\begin{array}{c}
\bar{n}_{L} \\
\bar{N}_{L} \\
\overline{\mathcal{N}}_{L}
\end{array}\right)^{T}\left(\begin{array}{ccc}
y_{u} v_{H} & \tilde{\lambda}_{u} v_{H} & m_{\ell_{L}} \\
\lambda_{u} v_{H} & \tilde{y}_{u} v_{H} & M_{\ell_{L}} \\
m_{\ell_{R}} & M_{\ell_{R}} & \tilde{y}_{u}^{\prime} v_{H}
\end{array}\right)\left(\begin{array}{c}
n_{R} \\
\mathcal{N}_{R} \\
N_{R}
\end{array}\right)+\text { H.c. } \\
&=\overline{\mathbf{u}}_{L} \mathcal{M}_{u} \mathbf{u}_{R}+\overline{\mathbf{d}}_{L} \mathcal{M}_{d} \mathbf{d}_{R}+\overline{\mathbf{e}}_{L} \mathcal{M}_{e} \mathbf{e}_{R}+\overline{\mathbf{n}}_{L} \mathcal{M}_{n} \mathbf{n}_{R}+\text { H.c., }
\end{aligned}
$$

where $\lambda_{u, d}, \tilde{\lambda}_{u, d}, y_{u, d} \tilde{\lambda}_{u, d}$ are the linear combinations of $\lambda_{1,2}, \tilde{\lambda}_{1,2}, y_{1,2}$ and $\tilde{y}_{1,2}$, respectively. ${ }^{3}$ The VEVs of the bi-doublet and adjoint are defined as

$$
\langle\Phi\rangle=v_{H} \times \operatorname{diag}\left(s_{\beta}, c_{\beta}\right), \quad\langle\Delta\rangle=\frac{v_{\Delta}}{2 \sqrt{3}} \times \operatorname{diag}(3,-1,-1,-1),
$$

where $s_{\beta}^{2}+c_{\beta}^{2}=1$ and $v_{H} \simeq 174 \mathrm{GeV}$. The mass matrices $M_{\mathcal{F}}$ and $m_{\mathcal{F}}\left(\mathcal{F}=Q_{L, R}, \ell_{L, R}\right)$ are defined as

$$
\begin{array}{ll}
M_{\ell_{L, R}}=M_{L, R}+\frac{3}{2 \sqrt{3}} \kappa_{L, R} v_{\Delta}, & M_{Q_{L, R}}=M_{L, R}-\frac{1}{2 \sqrt{3}} \kappa_{L, R} v_{\Delta}, \\
m_{\ell_{L, R}}=m_{L, R}+\frac{3}{2 \sqrt{3}} \epsilon_{L, R} v_{\Delta}, & m_{Q_{L, R}}=m_{L, R}-\frac{1}{2 \sqrt{3}} \epsilon_{L, R} v_{\Delta} .
\end{array}
$$

Here, $\mathcal{M}_{u}, \mathcal{M}_{d}, \mathcal{M}_{e}$ and $\mathcal{M}_{n}$ respectively represent $9 \times 9$ mass matrices for the nine generations of fermions $\mathbf{u}_{L, R}, \mathbf{d}_{L, R}$, $\mathbf{e}_{L, R}$ and $\mathbf{n}_{L, R}$. The Yukawa couplings with the SM Higgs bosons are the same for the down quarks and charged leptons, as well as the up quarks and neutrinos, while the vectorlike masses and Yukawa couplings with $\Delta$ are common in each of the quarks and leptons. Note that the orderings of the $S U(2)_{L}$ singlet and doublet states are flipped for which the electroweak (EW) gauge couplings are simplified. We define the mass basis for the charged fermions as

$$
\hat{\mathbf{u}}_{L, R}=\left(U_{L, R}^{u}\right)^{\dagger} \mathbf{u}_{L, R}, \quad \hat{\mathbf{d}}_{L, R}=\left(U_{L, R}^{d}\right)^{\dagger} \mathbf{d}_{L, R}, \quad \hat{\mathbf{e}}_{L, R}=\left(U_{L, R}^{e}\right)^{\dagger} \mathbf{e}_{L, R} .
$$

The unitary matrices diagonalize the mass matrices as

$$
\left(U_{L}^{f}\right)^{\dagger} \mathcal{M}_{f} U_{R}^{f}=\operatorname{diag}\left(m_{1}^{f}, m_{2}^{f}, \ldots, m_{9}^{f}\right),
$$

where $f=e, d, u$. The singular values are in ascending order, i.e., $m_{a}^{f} \leq m_{a+1}^{f}(a=1, \ldots, 8)$. The SM fermion masses are given by $m_{1}^{f}, m_{2}^{f}$ and $m_{3}^{f}$ :

\footnotetext{
${ }^{3}$ Their explicit relations are shown in Appendix A 3 .
} 


$$
\left(m_{1}^{e}, m_{2}^{e}, m_{3}^{e}\right)=\left(m_{e}, m_{\mu}, m_{\tau}\right), \quad\left(m_{1}^{d}, m_{2}^{d}, m_{3}^{d}\right)=\left(m_{d}, m_{s}, m_{b}\right), \quad\left(m_{1}^{u}, m_{2}^{u}, m_{3}^{u}\right)=\left(m_{u}, m_{c}, m_{t}\right) .
$$

The neutrino masses are explained by the type-I seesaw mechanism. Majorana masses for the right-handed neutrinos originate in Eq. (2.3). The scalar $\Sigma$ is represented as

$$
\Sigma_{\alpha \beta}=\Sigma_{\alpha \beta}^{k_{R}} \tau^{k_{R}}=\frac{1}{2}\left(\begin{array}{cc}
\Sigma_{\alpha \beta}^{3} & \sqrt{2} \Sigma_{\alpha \beta}^{+} \\
\sqrt{2} \Sigma_{\alpha \beta}^{-} & -\Sigma_{\alpha \beta}^{3}
\end{array}\right), \quad \Sigma_{\alpha \beta}^{ \pm}=\frac{\Sigma_{\alpha \beta}^{1} \pm i \Sigma_{\alpha \beta}^{2}}{\sqrt{2}},
$$

where $k_{R}=1,2,3$ is $S U(2)_{R}$ index and $\alpha, \beta=1,2,3,4$ are the $S U(4)_{C}$ indices. We shall assume that $\Sigma$ obtains the VEV as

$$
\left\langle\Sigma_{11}^{+}\right\rangle=\frac{v_{\Sigma}}{\sqrt{2}} \neq 0, \quad \text { others }=0
$$

where $\alpha, \beta=1$ is the leptonic direction in the $S U(4)_{C}$ space. After the symmetry breaking, the Majorana mass term is given by

$$
-\mathcal{L}_{\Sigma}^{y} \supset \frac{1}{2} \overline{\mathcal{N}}_{R}^{c} M_{R} \mathcal{N}_{R}+\text { H.c., } \quad M_{R}=\frac{h}{\sqrt{2}} v_{\Sigma} .
$$

The full neutrino mass term is given by

$$
-\mathcal{L}_{N}=\frac{1}{2} \overline{\mathbf{N}}_{L} \mathcal{M}_{N} \mathbf{N}_{R}:=\left(\begin{array}{ll}
\overline{\mathbf{n}}_{L} & \overline{\mathbf{n}}_{R}^{c}
\end{array}\right)\left(\begin{array}{cc}
0_{9} & \mathcal{M}_{n} \\
\mathcal{M}_{n}^{T} & \mathcal{M}_{R}
\end{array}\right)\left(\begin{array}{l}
\mathbf{n}_{L}^{c} \\
\mathbf{n}_{R}
\end{array}\right), \quad \mathcal{M}_{R}=\left(\begin{array}{ccc}
0_{3} & 0_{3} & 0_{3} \\
0_{3} & M_{R} & 0_{3} \\
0_{3} & 0_{3} & 0_{3}
\end{array}\right)
$$

where the Dirac mass matrix $\mathcal{M}_{n}$ is defined in Eq. (2.6). ${ }^{4}$ Note that $\mathbf{N}_{R}^{c}=\mathbf{N}_{L}$. The mass eigenstate is defined as

$$
\hat{\mathbf{N}}_{R}=U_{N}^{\dagger} \mathbf{N}_{R}, \quad \hat{\mathbf{N}}_{L}=U_{N}^{T} \mathbf{N}_{L}, \quad\left[U_{N}^{T} \mathcal{M}_{N} U_{N}\right]_{x y}=m_{\nu_{x}} \delta_{x y},
$$

with $x, y=1,2,3, \ldots, 18$. Here, $U_{N}$ is an $18 \times 18$ unitary matrix. We define the $9 \times 18$ matrices,

$$
U_{L}^{n}:=\mathcal{P}_{L} U_{N}^{*}, \quad U_{R}^{n}:=\mathcal{P}_{R} U_{N},
$$

where the projection matrices for the neutrino flavors are defined as

$$
\mathcal{P}_{L}:=\left(\begin{array}{ll}
\mathbf{1}_{9} & 0_{9}
\end{array}\right), \quad \mathcal{P}_{R}:=\left(\begin{array}{ll}
0_{9} & \mathbf{1}_{9}
\end{array}\right)
$$

\section{B. Leptoquark couplings}

The spontaneous PS gauge symmetry breaking generates the mass of the vector leptoquark, which is part of the $S U(4)_{C}$ gauge field. The gauge couplings with the vector leptoquark, $X^{\mu}$, are given by

$$
\begin{aligned}
\mathcal{L}_{X} & =\frac{g_{4}}{\sqrt{2}} X^{\mu}\left(\overline{\mathbf{d}}_{L} \gamma_{\mu} \mathbf{e}_{L}+\overline{\mathbf{u}}_{L} \gamma_{\mu} \mathbf{n}_{L}+\overline{\mathbf{d}}_{R} \gamma_{\mu} \mathbf{e}_{R}+\overline{\mathbf{u}}_{R} \gamma_{\mu} X^{\mu} \mathbf{n}_{R}\right)+\text { H.c. } \\
& =X^{\mu}\left(\hat{\mathbf{d}}_{L} \hat{g}_{d_{L}}^{X} \gamma_{\mu} \hat{\mathbf{e}}_{L}+\hat{\mathbf{u}}_{L} \hat{g}_{u_{L}}^{X} \gamma_{\mu} \hat{\mathbf{N}}_{L}+\hat{\overline{\mathbf{d}}}_{R} \hat{g}_{d_{R}}^{X} \gamma_{\mu} \hat{\mathbf{e}}_{R}+\hat{\mathbf{u}}_{R} \hat{g}_{u_{R}}^{X} \gamma_{\mu} \hat{\mathbf{N}}_{R}\right)+\text { H.c. }
\end{aligned}
$$

Here, the coupling matrices in the mass basis are given by

$$
\begin{array}{ll}
\hat{g}_{d_{L}}^{X}=\frac{g_{4}}{\sqrt{2}}\left(U_{L}^{d}\right)^{\dagger} U_{L}^{e}, & \hat{g}_{d_{R}}^{X}=\frac{g_{4}}{\sqrt{2}}\left(U_{R}^{d}\right)^{\dagger} U_{R}^{e}, \\
\hat{g}_{u_{L}}^{X}=\frac{g_{4}}{\sqrt{2}}\left(U_{L}^{u}\right)^{\dagger} U_{L}^{n}, & \hat{g}_{u_{R}}^{X}=\frac{g_{4}}{\sqrt{2}}\left(U_{R}^{u}\right)^{\dagger} U_{R}^{n} .
\end{array}
$$

The couplings of the fermions to the other gauge bosons and scalars are shown in Appendix A.

\footnotetext{
${ }^{4}$ In this paper, $0_{n}$ is an $n \times n$ zero matrix. Similarly, $\mathbf{1}_{n}$ is an $n \times n$ identity matrix.
} 
All the SM fermion masses and mixings can be explained consistently with the PS relations by the mass splittings via the Yukawa couplings with the bi-doublets $\Phi$ and adjoint $\Delta$ even if the $G_{\mathrm{PS}}$ breaking scale is at the $\mathrm{TeV}$ scale. The leptoquark, however, couples to the SM charged leptons and down-type quarks if the SM fermions dominantly come from the chiral fermions, $L$ and $R$. When the leptoquark has sizable couplings to the light flavor fermions, particularly electron and down-type quarks simultaneously, the measurement of the $K_{L} \rightarrow \mu e$ decay provides the most stringent bound on the leptoquark mass $[19,20] .{ }^{5}$ If $\hat{g}_{d_{L}}^{X}=\hat{g}_{d_{R}}^{X}=\left(g_{4} / \sqrt{2}\right) \cdot \mathbf{1}_{9}$, the branching fraction of $K_{L} \rightarrow \mu e$ is estimated as ${ }^{6}$

$\mathrm{BR}\left(K_{L} \rightarrow \mu e\right) \sim 1.4 \times 10^{-11} \times\left(\frac{1 \mathrm{PeV}}{m_{X}}\right)^{4}\left(\frac{g_{4}}{1.0}\right)^{4}$,

while the current upper bound is $4.7 \times 10^{-12}$. This implies that the leptoquark should be heavier than $1 \mathrm{PeV}$ in this case. So, if one wants to have a $\mathrm{TeV}$-scale leptoquark for, e.g., explanation of the current flavor anomalies, this constraint is a bottleneck. It is interesting that the leptoquark couplings to the light flavor fermions can be suppressed by introducing the vectorlike fermions in our model. In the following, we will show explicit mass matrices which are consistent with the $\mathrm{TeV}$-scale vector leptoquark, SM fermion mass and mixing. We will also see that most of the flavor violating processes as well as $K_{L} \rightarrow \mu e$ are sufficiently suppressed.

\section{How to suppress $K_{L} \rightarrow \mu e$}

We propose an explicit way to avoid the strong constraint from $K_{L} \rightarrow \mu e$ at the tree level, by tuning the vectorlike masses such that

$$
\begin{array}{ll}
m_{Q_{R}} \ll M_{Q_{R}}, & M_{Q_{L}} \ll m_{Q_{L}}, \\
M_{\ell_{R}} \ll m_{\ell_{R}}, & m_{\ell_{L}} \ll M_{\ell_{L}} .
\end{array}
$$

The mass matrices of the down quarks and charged leptons are schematically given by

$$
\begin{gathered}
\mathcal{M}_{d} \sim\left(\begin{array}{ccc}
0_{3} & \hat{m}_{e} & m_{Q_{L}} \\
\hat{m}_{d} & 0_{3} & 0_{3} \\
0_{3} & M_{Q_{R}} & 0_{3}
\end{array}\right), \\
\mathcal{M}_{e} \sim\left(\begin{array}{ccc}
0_{3} & \hat{m}_{e} & 0_{3} \\
\hat{m}_{d} & 0_{3} & M_{\ell_{L}} \\
m_{\ell_{R}} & 0_{3} & 0_{3}
\end{array}\right),
\end{gathered}
$$

\footnotetext{
${ }^{5}$ We note that the flavor constraints from $K \rightarrow \pi \mu e$ are milder than the one obtained from $K_{L} \rightarrow \mu e$ [87].

${ }^{6}$ See Eq. (B1) for the full formula.
}

where $\hat{m}_{d}$ and $\hat{m}_{e}$ are the $3 \times 3$ mass matrices proportional to $v_{H}$. The same structure will arise in the up quark and neutrino sector, given the hierarchy in Eq. (2.22). With this texture, $\hat{m}_{d}$ and $\hat{m}_{e}$ approximately correspond to the mass matrices for the SM down-type quarks and charged leptons since the mixing with the other blocks is suppressed. Thus, the masses and mixing of the SM fermions can be explained separately. The down quarks originate in $\left(F_{L}, R\right)$, while the charged leptons in $\left(L, f_{R}\right)$. In this case, at least one of the two fermions involved in the leptoquark interaction [Eq. (2.19)] is not the SM one, implying that the leptoquark does not mediate the rare meson decays at the tree level. The nonzero leptoquark couplings to two SM fermions arise as the condition of Eq. (2.22) is relaxed, as we will discuss in the next section.

It should be noted that the mass structure in Eq. (2.23) suppresses the $K_{L} \rightarrow \mu e$ transition at the tree level, so it may be induced at loop levels. For instance, the loop diagrams involving the vectorlike fermions, leptoquark and $\Delta$ scalar may give a sizable contribution. The analysis with the loop contribution is interesting, but beyond the scope of this paper. In the following, we focus on the tree-level contribution assuming loop corrections are subdominant because of e.g., sufficiently heavy $\Delta$ scalar and/or small coupling constants.

\section{Parametrization}

In our notation, it is convenient to express the mass matrices by a $6 \times 6$ block on the upper left and a $3 \times 3$ block on the bottom right. Without loss of generality, ${ }^{7}$ we can parametrize the Dirac mass matrices in the gauge basis as

$$
\begin{aligned}
\mathcal{M}_{d} & =\left(\begin{array}{cc}
\tilde{D}_{d} & V_{Q_{L}} \tilde{D}_{Q_{L}} W_{Q_{R}}^{\dagger} \\
W_{Q_{L}} \tilde{D}_{Q_{R}} V_{Q_{R}}^{\dagger} & \Delta_{d}
\end{array}\right), \\
\mathcal{M}_{e} & =\left(\begin{array}{cc}
\tilde{D}_{d} & V_{\ell_{L}} \tilde{D}_{\ell_{L}} W_{\ell_{R}}^{\dagger} \\
W_{\ell_{L}} \tilde{D}_{\ell_{R}} V_{\ell_{R}}^{\dagger} & \Delta_{d}
\end{array}\right), \\
\mathcal{M}_{u} & =\left(\begin{array}{cc}
v_{u_{L}} \tilde{D}_{u} v_{u_{R}}^{\dagger} & V_{Q_{L}} \tilde{D}_{Q_{L}} W_{Q_{R}}^{\dagger} \\
W_{Q_{L}} \tilde{D}_{Q_{R}} V_{Q_{R}}^{\dagger} & w_{u_{L}} \Delta_{u} w_{u_{R}}^{\dagger}
\end{array}\right), \\
\mathcal{M}_{n} & =\left(\begin{array}{cc}
v_{u_{L}} \tilde{D}_{u} v_{u_{R}}^{\dagger} & V_{\ell_{L}} \tilde{D}_{\ell_{L}} W_{\ell_{R}}^{\dagger} \\
W_{\ell_{L}} \tilde{D}_{\ell_{R}} V_{\ell_{R}}^{\dagger} & w_{u_{L}} \Delta_{u} w_{u_{R}}^{\dagger}
\end{array}\right),
\end{aligned}
$$

where

$$
\tilde{D}_{d}=\left(\begin{array}{cc}
0_{3} & D_{e} \\
D_{d} & 0_{3}
\end{array}\right), \quad \tilde{D}_{u}=\left(\begin{array}{cc}
0_{3} & D_{n} \\
D_{u} & 0_{3}
\end{array}\right)
$$

\footnotetext{
${ }^{7}$ Here, the mass matrices are expressed by the singular value decomposition, as usually applied to the Dirac mass matrix.
} 
and

$$
\tilde{D}_{Q_{R}}=\left(\begin{array}{cc}
0_{3} & D_{Q_{R}}
\end{array}\right), \quad \tilde{D}_{Q_{L}}=\left(\begin{array}{c}
D_{Q_{L}} \\
0_{3}
\end{array}\right), \quad \tilde{D}_{\ell_{R}}=\left(\begin{array}{ll}
D_{\ell_{R}} & 0_{3}
\end{array}\right), \quad \tilde{D}_{\ell_{L}}=\left(\begin{array}{c}
0_{3} \\
D_{\ell_{L}}
\end{array}\right) .
$$

Here, $D_{f}(f=u, d, e, n)$ and $D_{F_{L, R}}(F=Q, \ell)$ are $3 \times 3$ diagonal matrices. There are four $6 \times 6$ unitary matrices $V_{Q_{L, R}}$, $V_{\ell_{L, R}}$ and eight $3 \times 3$ unitary matrices $W_{Q_{L, R}}, W_{\ell_{L, R}}$ and $v_{u_{L, R}}, w_{u_{L, R}}$. By definition, the mass matrices are unchanged under

$$
V_{\ell_{L, R}} \rightarrow\left(\begin{array}{cc}
u_{\ell_{L, R}} & 0_{3} \\
0_{3} & \mathbf{1}_{3}
\end{array}\right) V_{\ell_{L, R}}, \quad V_{Q_{L, R}} \rightarrow\left(\begin{array}{cc}
u_{Q_{L, R}} & 0_{3} \\
0_{3} & \mathbf{1}_{3}
\end{array}\right) V_{Q_{L, R}},
$$

where $u_{F_{L, R}}$ are arbitrary $3 \times 3$ unitary matrices. We start from a basis in which $D_{d}, D_{e}$ and $\Delta_{d}$ are diagonalized, which can be done without changing any couplings with the gauge bosons or scalars. See Appendix A 1 for more details. We further assume that the Majorana matrix $M_{R}$ is proportional to an identity matrix in this basis for simplicity.

This parametrization is defined such that the SM-like fermion masses and couplings are realized when all the unitary matrices are identity matrices except $v_{u_{L}}$ which should be

$$
v_{u_{L}}=\left(\begin{array}{cc}
U_{\mathrm{PMNS}} & 0_{3} \\
0_{3} & V_{\mathrm{CKM}}^{\dagger}
\end{array}\right),
$$

so that the Cabbibo-Kobayashi-Maskawa (CKM) and Pontecorvo-Maki-Nakagawa-Sakata (PMNS) matrices are realized. In this canonical case, the (Dirac) mass matrices are diagonalized by the following unitary matrices:

$$
\begin{array}{ll}
U_{L}^{0, d}=U_{R}^{0, e}=U_{R}^{0, n}=\left(\begin{array}{lll}
0_{3} & \mathbf{1}_{3} & 0_{3} \\
\mathbf{1}_{3} & 0_{3} & 0_{3} \\
0_{3} & 0_{3} & \mathbf{1}_{3}
\end{array}\right), & U_{L}^{0, u}=\left(\begin{array}{ccc}
0_{3} & \mathbf{1}_{3} & 0_{3} \\
V_{\mathrm{CKM}}^{\dagger} & 0_{3} & 0_{3} \\
0_{3} & 0_{3} & \mathbf{1}_{3}
\end{array}\right), \\
U_{R}^{0, u}=U_{R}^{0, d}=U_{L}^{0, e}=\left(\begin{array}{lll}
\mathbf{1}_{3} & 0_{3} & 0_{3} \\
0_{3} & 0_{3} & \mathbf{1}_{3} \\
0_{3} & \mathbf{1}_{3} & 0_{3}
\end{array}\right), & U_{L}^{0, n}=\left(\begin{array}{ccc}
U_{\mathrm{PMNS}} & 0_{3} & 0_{3} \\
0_{3} & 0_{3} & \mathbf{1}_{3} \\
0_{3} & \mathbf{1}_{3} & 0_{3}
\end{array}\right),
\end{array}
$$

up to $\mathcal{O}\left(v_{H} / v_{\Delta}\right)$ corrections. For the full neutrino matrix, the diagonalization matrix is given by

$$
U_{N}^{0} \sim\left(\begin{array}{cc}
\left(U_{L}^{0, n}\right)^{*} & 0_{9} \\
0_{9} & U_{R}^{0, n}
\end{array}\right)
$$

Then, the leptoquark couplings are approximately given by

$$
\hat{g}_{d_{L}}^{X} \sim\left(\hat{g}_{d_{R}}^{X}\right)^{T} \sim \mathcal{P}_{R}\left(\hat{g}_{u_{R}}^{X}\right)^{T} \sim \frac{g_{4}}{\sqrt{2}}\left(\begin{array}{ccc}
0_{3} & 0_{3} & \mathbf{1}_{3} \\
\mathbf{1}_{3} & 0_{3} & 0_{3} \\
0_{3} & \mathbf{1}_{3} & 0_{3}
\end{array}\right), \quad \hat{g}_{u_{L}}^{X} \mathcal{P}_{L}^{T} \sim \frac{g_{4}}{\sqrt{2}}\left(\begin{array}{ccc}
0_{3} & 0_{3} & V_{\mathrm{CKM}} \\
U_{\mathrm{PMNS}} & 0_{3} & 0_{3} \\
0_{3} & \mathbf{1}_{3} & 0_{3}
\end{array}\right) .
$$

Now, the leptoquark couplings to the SM fermions, which correspond to the most upper-left block in the coupling matrix, e.g., $\left[\hat{g}_{d_{L}}^{X}\right]_{i j}(i, j \leq 3)$, are vanishing. Thus, in this canonical case, there is no flavor violation at the tree level, although there might be flavor violation, such as $\mu \rightarrow e \gamma$, from the loop effects involving the vectorlike states. In order to explain the flavor anomalies, the leptoquark should couple to the SM fermions with a certain pattern. In the following, we will turn on the mixing angles in the unitary matrices which are chosen to be the identity matrices in the canonical limit. The diagonalization for a general case with the parametrization in Eq. (2.24) is discussed in Appendix A 1.

For simplicity, we assume that the singular values for vectorlike fermions and $\Delta_{u, d}$ are universal, ${ }^{8}$ i.e.,

$$
D_{Q_{L}}=d_{Q_{L}} \mathbf{1}_{3}, \quad D_{Q_{R}}=d_{Q_{R}} \mathbf{1}_{3}, \quad D_{\ell_{L}}=d_{\ell_{L}} \mathbf{1}_{3}, \quad D_{\ell_{R}}=d_{\ell_{R}} \mathbf{1}_{3},
$$

\footnotetext{
${ }^{8}$ Precisely, we introduce $\mathcal{O}(0.1 \mathrm{GeV})$ corrections to the vectorlike fermion masses to avoid numerical instabilities due to the degeneracy as can be seen in Appendix B.
} 
and

$$
\Delta_{d}=\delta_{d} \mathbf{1}_{3}, \quad \Delta_{u}=\delta_{u} \mathbf{1}_{3}
$$

Here, the mass parameters in Eq. (2.32) are $\mathcal{O}\left(v_{\Delta}\right)$, while those in Eq. (2.33) are $\mathcal{O}\left(v_{H}\right)$. We also assume that the Majorana mass matrix is given by

$$
\mathcal{M}_{R}=m_{N}\left(\begin{array}{lll}
0_{3} & 0_{3} & 0_{3} \\
0_{3} & \mathbf{1}_{3} & 0_{3} \\
0_{3} & 0_{3} & 0_{3}
\end{array}\right) \text {. }
$$

Further, we take $W_{Q_{L, R}}, W_{\ell_{L, R}}$ and $w_{u_{L, R}}$ identity matrices, since these are not significant for the SM fermion couplings.

When the $V_{Q_{L, R}}$ and $V_{\ell_{L, R}}$ are not identity matrices, the diagonalization matrices for the down-type fermions in Eq. (2.24) are approximately given by

$$
\begin{array}{ll}
U_{e_{L}} \sim\left(\begin{array}{cc}
0_{6 \times 3} & V_{\ell_{L}} \\
W_{\ell_{L}} & 0_{3 \times 6}
\end{array}\right) P_{1}, & U_{e_{R}} \sim\left(\begin{array}{cc}
V_{\ell_{R}} & 0_{6 \times 3} \\
0_{3 \times 6} & W_{\ell_{R}}
\end{array}\right) P_{1}, \\
U_{d_{L}} \sim\left(\begin{array}{cc}
V_{Q_{L}} & 0_{6 \times 3} \\
0_{3 \times 6} & W_{Q_{L}}
\end{array}\right) P_{1}, & U_{d_{R}} \sim\left(\begin{array}{cc}
0_{6 \times 3} & V_{Q_{R}} \\
W_{Q_{R}} & 0_{3 \times 6}
\end{array}\right) P_{1},
\end{array}
$$

where

$$
P_{1}:=\left(\begin{array}{lll}
0_{3} & \mathbf{1}_{3} & 0_{3} \\
\mathbf{1}_{3} & 0_{3} & 0_{3} \\
0_{3} & 0_{3} & \mathbf{1}_{3}
\end{array}\right)
$$

is introduced so that the singular values are increasingly ordered. The derivation and those for the up-type fermions are shown in Appendix A 1. With this parametrization, the leptoquark couplings to the SM fermions are approximately given by

$$
\begin{aligned}
& {\left[\hat{g}_{d_{L}}^{X}\right]_{i j} \sim\left[\hat{g}_{u_{L}}^{X}\right]_{i j} \sim \frac{g_{4}}{\sqrt{2}}\left[V_{Q_{L}}^{\dagger} V_{\ell_{L}}\right]_{3+i, j},} \\
& {\left[\hat{g}_{d_{R}}^{X}\right]_{i j} \sim\left[\hat{g}_{u_{R}}^{X}\right]_{i j} \sim \frac{g_{4}}{\sqrt{2}}\left[V_{Q_{R}}^{\dagger} V_{\ell_{R}}\right]_{i, j+3},}
\end{aligned}
$$

with $i, j=1,2,3$. Hence, the leptoquark couplings to the SM families are induced through the mixing between the first three and the second three states. We parametrize the unitary matrices, $V_{F_{X}}, F=\ell, Q, X=L, R$, as ${ }^{9}$

$$
V_{F_{X}}:=R_{F_{X}}^{11} R_{F_{X}}^{12} R_{F_{X}}^{13} R_{F_{X}}^{21} R_{F_{X}}^{22} R_{F_{X}}^{23} R_{F_{X}}^{31} R_{F_{X}}^{32} R_{F_{X}}^{33},
$$

\footnotetext{
${ }^{9}$ Here, we assumed the matrices are real for simplicity.
}

where the rotation matrix $R_{F_{X}}^{i j}$ mixes the $i$ th and $(j+3)$ th elements, i.e.,

$$
R_{F_{X}}^{i j}=\left(\begin{array}{ccccccc}
1 & \cdots & 0 & \cdots & 0 & \cdots & 0 \\
\vdots & & \vdots & & \vdots & & \vdots \\
0 & \cdots & c_{F_{X}}^{i j} & \cdots & s_{F_{X}}^{i j} & \cdots & 0 \\
\vdots & & \vdots & & \vdots & & \vdots \\
0 & \cdots & -s_{F_{X}}^{i j} & \cdots & c_{F_{X}}^{i j} & \cdots & 0 \\
\vdots & & \vdots & & \vdots & & \vdots \\
0 & \cdots & 0 & \cdots & 0 & \cdots & 1
\end{array}\right),
$$

where $\left(c_{F_{X}}^{i j}\right)^{2}+\left(s_{F_{X}}^{i j}\right)^{2}=1$. In our analysis, we assume that $V_{F_{X}}$ 's are real and we will not consider the mixing inside the first three and second three states.

In our numerical analysis of Sec. III, the vectorlike fermion masses $d_{Q_{L, R}}, d_{\ell_{L, R}}, \delta_{u, d}$ and the unitary matrices $V_{F_{X}}$ are input parameters. The rest of the parameters, $\tilde{D}_{d}$, $\tilde{D}_{u}$ and $v_{u_{L}}, v_{u_{R}}$ are fitted to explain the observed fermion masses and mixing, given a set of input parameters.

\section{E. Fine-tuning}

The texture in Eq. (2.23), or the hierarchy in Eq. (2.22), requires fine-tunings between the PS symmetric mass parameters in the first line of Eq. (2.2) and the mass terms originated from the $S U(4)_{C}$ adjoint $\langle\Delta\rangle$. To quantify the degree of tunings, we define the tuning measure $\Delta_{\mathrm{FT}}$ as

$$
\Delta_{\mathrm{FT}}:=\min _{A, B}\left(\left[\Delta_{d_{L}}\right]_{A},\left[\Delta_{d_{R}}\right]_{B}\right),
$$

where

$$
\begin{aligned}
{\left[\Delta_{d_{L}}\right]_{A} } & :=\frac{\min \left(\left|\left[\mathcal{M}_{d}\right]_{A, 6+\tilde{A}}\right|,\left|\left[\mathcal{M}_{e}\right]_{A, 6+\tilde{A}}\right|\right)}{\max \left(\left|\left[\mathcal{M}_{d}\right]_{A, 6+\tilde{A}}\right|,\left|\left[\mathcal{M}_{e}\right]_{A, 6+\tilde{A}}\right|\right)}, \\
{\left[\Delta_{d_{R}}\right]_{B} } & :=\frac{\min \left(\left|\left[\mathcal{M}_{d}\right]_{6+B, \tilde{B}}\right|,\left|\left[\mathcal{M}_{e}\right]_{6+B, \tilde{B}}\right|\right)}{\max \left(\left|\left[\mathcal{M}_{d}\right]_{6+B, \tilde{B}}\right|,\left|\left[\mathcal{M}_{e}\right]_{6+B, \tilde{B}}\right|\right)} .
\end{aligned}
$$

Here, $A, B=1,2,3, \ldots, 6$ and $\tilde{A}, \tilde{B}$ are respectively residues of $A, B$ divided by 3 , so that it measures the degree of cancellations in the diagonal elements of the vectorlike masses. For instance, $10 \%$ tuning is required if $\Delta_{\mathrm{FT}}$ is 0.1 .

\section{PHENOMENOLOGY}

We study phenomenology in this setup. We have seen the way to suppress $K_{L} \rightarrow \mu e$. The suppression, however, may require severe fine-tuning. If the fine-tuning is relaxed, other flavor violating processes would become sizable. First of all, we discuss the possibility that the TeV-scale leptoquark explains the anomalies in the semileptonic $B$ 
meson decays. Then, we investigate the other flavor violating processes paying attention to the degree of tuning.

\section{A. Vector leptoquark explanation of the anomalies}

Interestingly, the $\mathrm{TeV}$-scale leptoquark may be able to explain all anomalies in the semileptonic $B$ meson decays, $B \rightarrow K^{(*)} \mu \mu$ and $B \rightarrow D^{(*)} \tau \nu$. It has been pointed out in the literature that the vector leptoquark from the PS model can explain both anomalies simultaneously, but we show that the $b \rightarrow c \tau \nu$ anomaly is hardly solved in our model due to the correlation between the leptoquark and $Z^{\prime}$ boson masses. ${ }^{10}$

\section{1. $b \rightarrow s \mu \mu$ anomaly}

The $b \rightarrow s \mu \mu$ anomaly may be a signal of new physics. The effective Hamiltonian is given by

$$
\mathcal{H}_{\mathrm{eff}}^{b \rightarrow s \mu \mu}=-\frac{4 G_{F}}{\sqrt{2}} \frac{\alpha}{4 \pi} \sum_{a=9,10}\left(C_{a} \mathcal{O}_{a}+C_{a}^{\prime} \mathcal{O}_{a}^{\prime}\right)
$$

where

$$
\mathcal{O}_{9}=\left(\bar{s} \gamma^{\rho} P_{L} b\right)\left(\bar{\mu} \gamma_{\rho} \mu\right), \quad \mathcal{O}_{10}=\left(\bar{s} \gamma^{\rho} P_{L} b\right)\left(\bar{\mu} \gamma_{\rho} \gamma_{5} \mu\right),
$$

$$
\mathcal{O}_{9}^{\prime}=\left(\bar{s} \gamma^{\rho} P_{R} b\right)\left(\bar{\mu} \gamma_{\rho} \mu\right), \quad \mathcal{O}_{10}^{\prime}=\left(\bar{s} \gamma^{\rho} P_{R} b\right)\left(\bar{\mu} \gamma_{\rho} \gamma_{5} \mu\right) .
$$

The leptoquark contributions to the Wilson coefficients, $C_{9}$ and $C_{10}$, are given by

$$
\begin{aligned}
\Delta C_{9}= & -\Delta C_{10}=-\frac{\sqrt{2}}{4 G_{F}} \frac{4 \pi}{\alpha} \frac{1}{V_{t b}^{*} V_{t s}} \frac{1}{2 m_{X}^{2}}\left[\hat{g}_{d_{L}}^{X}\right]_{32}^{*}\left[\hat{g}_{d_{L}}^{X}\right]_{22} \\
& \sim-0.51 \times\left(\frac{5 \mathrm{TeV}}{m_{X}}\right)^{2}\left(\frac{\left[\hat{g}_{d_{L}}^{X}\right]_{32}^{*}\left[\hat{g}_{d_{L}}^{X}\right]_{22}}{0.02}\right) .
\end{aligned}
$$

The experimental results favor $-0.59 \lesssim \Delta C_{9} \lesssim-0.41$ at $1 \sigma$ level [89], and thus $m_{X} \sim \mathcal{O}(10 \mathrm{TeV})$ is a suitable size to explain the $b \rightarrow s \mu \mu$ anomaly. Note that there exists a $Z^{\prime}$ boson in our model which is associated with the PS symmetry breaking. Such a boson would contribute to the $b \rightarrow s \mu \mu$ anomaly. However, the flavor violating effects from the $Z^{\prime}$ boson are expected to be very suppressed, as discussed in Appendix A 2.

With the parametrization of Eq. (2.39), the relevant couplings are given by

\footnotetext{
${ }^{10}$ The correlation between leptoquark and $Z^{\prime}$ has been also studied in a more generic way [88].
}

$$
\begin{aligned}
& {\left[\hat{g}_{d_{L}}^{X}\right]_{22}=\frac{g_{4}}{\sqrt{2}} c_{\ell_{L}}^{23}\left(s_{Q_{L}}^{22} c_{\ell_{L}}^{22}-c_{Q_{L}}^{22} s_{\ell_{L}}^{22}\right),} \\
& {\left[\hat{g}_{d_{L}}^{X}\right]_{32}=\frac{g_{4}}{\sqrt{2}}\left\{s_{Q_{L}}^{23} c_{\ell_{L}}^{23}\left(c_{Q_{L}}^{22} c_{\ell_{L}}^{22}+s_{Q_{L}}^{22} s_{\ell_{L}}^{22}\right)-s_{\ell_{L}}^{23} c_{Q_{L}}^{23}\right\},}
\end{aligned}
$$

if the angles only in $R_{Q_{L}}^{22}, R_{Q_{L}}^{23}, R_{\ell_{L}}^{22}$ and $R_{\ell_{L}}^{23}$ are turned on. We fix the angles at

$s_{Q_{L}}^{23}=-s_{\ell_{L}}^{23}=\frac{1}{\sqrt{2}}, \quad s_{Q_{L}}^{22}=-s_{\ell_{L}}^{22}=0.04 \times \frac{1}{\sqrt{2}}$,

so that the $b \rightarrow s \mu \mu$ anomaly is explained with $m_{X} \sim 5 \mathrm{TeV}$ and $\left|\left[\hat{g}_{d_{L}}^{X}\right]_{22} /\left[\hat{g}_{d_{L}}^{X}\right]_{23}\right| \sim 0.04$. As discussed later, $\left[\hat{g}_{d_{L}}^{X}\right]_{22}$ should be small to suppress $K_{L} \rightarrow \mu e$. Note that this model, in general, predicts the leptoquark contributions to the other lepton flavors as well as $C_{9,10}^{\prime}$. In our analysis, we will consider the parameter space where these are negligibly small.

\section{2. $b \rightarrow c \tau \nu$ anomaly}

The effective Hamiltonian relevant to $b \rightarrow c \tau \nu$ within our model is given by

$$
\mathcal{H}_{\mathrm{eff}}^{b \rightarrow c \tau \nu}=\frac{4 G_{F}}{\sqrt{2}} V_{c b} C_{V_{1}}\left(\bar{c} \gamma^{\mu} P_{L} b\right)\left(\bar{\tau} P_{L} \nu_{\tau}\right) .
$$

The leptoquark contribution to the Wilson coefficients $C_{V_{1}}$ is given by

$$
\begin{aligned}
\Delta C_{V_{1}}= & \frac{\sqrt{2}}{4 G_{F}} \frac{1}{V_{c b}} \frac{1}{m_{X}^{2}}\left[\hat{g}_{d_{L}}^{X}\right]_{33}^{*}\left[\hat{g}_{u_{L}}^{X}\right]_{2 \nu} \\
& \sim 0.092 \times\left(\frac{1.4 \mathrm{TeV}}{m_{X}}\right)^{2}\left(\frac{\left.\hat{g}_{d_{L}}^{X}\right]_{33}^{*}\left[\hat{g}_{u_{L}}^{X}\right]_{2 \nu}}{0.25}\right),
\end{aligned}
$$

where $\left[\hat{g}_{u_{L}}^{X}\right]_{2 \nu}:=\sum_{i=1,2,3}\left[\hat{g}_{u_{L}}^{X}\right]_{2 i}$. The experimental results favor $0.052 \lesssim \Delta C_{V_{1}} \lesssim 0.124$ at $2 \sigma$ level [90]. Note that $\left[\hat{g}_{d_{L}}^{X}\right]_{33}^{*}\left[\hat{g}_{d_{L}}^{X}\right]_{2 \nu} \sim g_{4}^{2} / 4 \sim 0.25$ is the maximal value as far as the $S U(4)_{C}$ gauge coupling constant is $g_{4} \sim 1.0$ which is consistent with the strong coupling constant at the $\mathrm{TeV}$ scale [91]. Thus, $\Delta C_{V_{1}} \sim 0.09$ (0.057) could be explained if $m_{X} \sim 1.4(1.8) \mathrm{TeV}$.

The LHC result [92] searching for di-lepton resonance severely constrains a $Z^{\prime}$ boson mass if the $Z^{\prime}$ boson decays to a pair of electrons or muons. In our model, the $Z^{\prime}$ boson couples to the fermions in the similar way as the $Z$ boson. Therefore the resonant production cross section via the Drell-Yan process can be large as long as $Z^{\prime}$ boson is light, and there are sizable branching fractions to di-leptons, as we see in Eq. (A60) of Appendix A 2. If all the vectorlike fermions are heavier than half of the $Z^{\prime}$ mass, the limit is about $5 \mathrm{TeV}$. The limit is relaxed to about $4.5 \mathrm{TeV}$ if the $Z^{\prime}$ boson can decay to vectorlike fermions, since the branching 
fractions to di-leptons are suppressed by $1 / 3 .^{11}$ From Eqs. (A22) and (A37) in Appendix A 2, the $Z^{\prime}$ boson mass is bounded by the leptoquark mass,

$m_{Z^{\prime}}<\sqrt{\frac{2 g_{R}^{2}+3 g_{4}^{2}}{g_{4}^{2}}} m_{X} \sim 3.5 \mathrm{TeV} \times\left(\frac{m_{X}}{1.8 \mathrm{TeV}}\right)$,

where the upper bound is saturated when $v_{\Delta}=0$. Hence, when the leptoquark is light enough to address the $b \rightarrow c \tau \nu$ anomaly, the $Z^{\prime}$ boson is too light to be consistent with the current limit in the collider search. Furthermore, the vectorlike fermions will have masses of $\mathcal{O}\left(v_{\Delta}\right)$ unless the Yukawa couplings are nonperturbatively large. The LHC limit on a single vectorlike quark is about $1.2 \mathrm{TeV}$ when the vectorlike quark decays to a SM boson and a quark in the third generation [93]. Thus, the vector leptoquark explanation of the $b \rightarrow c \tau \nu$ anomaly is excluded by the $Z^{\prime}$ and vectorlike quark searches. We note that it would be possible that the $b \rightarrow c \tau \nu$ anomaly is explained in the scalar leptoquarks in PS models as studied in e.g., Ref. [94].

\section{B. Flavor physics}

We shall discuss the flavor physics induced by the leptoquark and the extra Higgs bosons when $m_{X}=$ $5 \mathrm{TeV}$ and the sizable leptoquark couplings are given by Eqs. (3.5) and (3.6), so that the $b \rightarrow s \mu \mu$ anomaly is explained. The formulas and values of constants used in our numerical analysis are shown in Appendix B.

In this subsection, we study our predictions at the tree level in flavor physics, except for $\mu \rightarrow e \gamma$. We do not include tree-level contributions of the $S U(4)_{C}$ adjoint $\Delta$ and $S U(2)_{R}$ triplet $\Sigma$. As shown in Appendix A 3, the adjoint $\Delta$ does not couple to two SM fermions up to $\mathcal{O}\left(v_{H} / v_{\Delta}\right)$. Hence tree-level processes induced by those scalars are very suppressed. As mentioned above, the loop corrections involving both scalars and leptoquark possibly induce flavor violating processes, such as $K_{L} \rightarrow \mu e$, even if all $s_{F_{X}}^{i j}$ are vanishing. We will neglect such contributions assuming the scalars involved in the loop are so heavy and/ or relevant couplings are so small that the loop corrections are subdominant. For $\Sigma$, it is shown in [94] that the scalar leptoquarks from it can explain both $b \rightarrow s \mu \mu$ and $b \rightarrow c \tau \nu$ anomalies as well as inducing various flavor violations if one considers a left-right symmetric extension of the PS model. We will study the neutrino antineutrino oscillation induced by $\Sigma$ in Sec. III C. At least in the canonical limit, only the charged leptons among the SM fermions couple to the scalar fields in $\Sigma$ at the tree level, but there is no flavor violation as ensured by the simplified structure of the Majorana mass matrix, Eq. (2.34). The couplings to light flavor leptons could be suppressed if the Majorana mass

\footnotetext{
${ }^{11}$ The larger decay width will also relax the limit.
}

matrix has a hierarchical structure, while the sizable flavor violation could be induced if the Majorana mass matrix has an off-diagonal element. The phenomenology of those scalar fields may be the interesting subject, but this is beyond the scope of this paper. Hereafter, we simply assume that the flavor violating effects via these scalars are negligible and we will focus on the physics of the vector leptoquark and the Higgs doublets.

\section{1. $\mu$-e flavor violation versus fine-tuning}

In our model, the stringent constraints from the rare meson decays can be avoided due to the texture of Eq. (2.23) which is achieved by fine-tuning the vectorlike mass terms such that there are cancellations between the mass parameters and the adjoint $\operatorname{VEV}\langle\Delta\rangle$, see Eq. (2.9). To quantify how accurately this cancellation should be held, we turn on the angles universally except for those relevant to the $b \rightarrow s \mu \mu$ anomaly,

$s_{Q_{L}}:=s_{q_{L}}^{\hat{i} \hat{j}}, \quad s_{Q_{R}}:=s_{q_{R}}^{i j}, \quad s_{\ell_{R}}:=s_{\ell_{L} \hat{i} \hat{j}}, \quad s_{\ell_{R}}:=s_{\ell_{R}}^{i j}$,

where $i, j=1,2,3$ run over all the combinations and $\hat{i}, \hat{j}=1,2,3$ also run over all the combinations except $(\hat{i}, \hat{j})=(2,2),(2,3)$, to keep $\Delta C_{9}$ unchanged approximately. The nonvanishing angles induce the flavor violating leptoquark couplings. Below, we will focus on $\mu-e$ flavor violating processes that will provide the most severe constraint on our model.

Turning on the mixing angles induces the leptoquark couplings to the first generation fermions. The induced couplings generate the $K_{L} \rightarrow \mu e$ decay. The branching fraction is approximately given by

$$
\begin{aligned}
& \operatorname{BR}\left(K_{L} \rightarrow \mu e\right) \\
& \sim \frac{\tau_{K_{L}}}{128 \pi m_{X}^{4}} \frac{m_{K}^{5} f_{K}^{2}}{m_{s}^{2}}\left(1-\frac{m_{\mu}^{2}}{m_{K}^{2}}\right)^{2}\left|\left[\hat{g}_{d_{L}}^{X}\right]_{22}\left[\hat{g}_{d_{R}}^{X}\right]_{11}\right|^{2} \\
& \sim 2.3 \times 10^{-12} \times\left(\frac{5 \mathrm{TeV}}{m_{X}}\right)^{4}\left(\frac{\left.\mid \hat{g}_{d_{L}}^{X}\right]_{22}\left[\hat{g}_{d_{R}}^{X}\right]_{11} \mid}{10^{-5}}\right)^{2} .
\end{aligned}
$$

Here we assume that $\left[\hat{g}_{d_{L}}^{X}\right]_{22}$ is much larger than the other couplings except for those involving the third generation. The experimental upper bound on the branching ratio is $4.7 \times 10^{-12}$ [95].

The leptoquark coupling will be also strongly constrained by the measurements of the $\mu-e$ conversion for an aluminum (gold) target in the future (current) experiments. The conversion per capture rate for an aluminum target is approximately given by 


$$
\begin{aligned}
\operatorname{BR}(\mu \rightarrow e)^{\mathrm{Al}} & \sim \frac{16 m_{\mu}^{5} S_{p}^{2} m_{p}^{2}}{m_{X}^{4} \Gamma_{\text {capt }}}\left|\frac{f_{S_{p}}^{s}}{m_{s}}\left[\hat{g}_{d_{R}}\right]_{21}^{*}\left[\hat{g}_{d_{L}}\right]_{22}+\frac{2}{27} \frac{f_{G}^{p}}{m_{b}}\left[\hat{g}_{d_{R}}\right]_{31}^{*}\left[\hat{g}_{d_{L}}\right]_{32}\right|^{2} \\
& \sim 1.5 \times 10^{-14} \times\left(\frac{5 \mathrm{TeV}}{m_{X}}\right)^{2}\left|0.45\left(\frac{\left[\hat{g}_{d_{R}}\right]_{21}^{*}\left[\hat{g}_{d_{L}}\right]_{22}}{10^{-5}}\right)+0.016\left(\frac{\left[\hat{g}_{d_{R}}\right]_{31}^{*}\left[\hat{g}_{d_{L}}\right]_{32}}{10^{-5}}\right)\right|^{2},
\end{aligned}
$$

where the mass and form factors for the neutron are set to be those for the proton for simplicity in the second line. This rate is the same order of magnitude for the gold target. The future (current) limit on the conversion rate per capture rate is [96-99]

$$
\mathrm{BR}(\mu \rightarrow e)^{\mathrm{Al}(\mathrm{Au})}=\frac{\Gamma_{\text {conv }}}{\Gamma_{\text {capt }}}<6 \times 10^{-17} \quad\left(7 \times 10^{-13}\right) .
$$

We note that even if the $K_{L} \rightarrow \mu e$ is sufficiently suppressed, the $\mu-e$ conversion rate can be larger than the future sensitivity because of the contribution from the other coupling parameters.

There may be constraints from $\mu \rightarrow e \gamma$ induced by the loop effects mediated by the leptoquarks and vectorlike down quarks. The branching fraction is given by [100]

$$
\mathrm{BR}(\mu \rightarrow e \gamma)=\tau_{\mu} \frac{\alpha_{e} m_{\mu}^{3}}{1024 \pi^{4} m_{X}^{4}}\left(\left|C_{L}^{\mu e}\right|^{2}+\left|C_{R}^{\mu e}\right|^{2}\right),
$$

where $\tau_{\mu}$ denotes the muon lifetime and

$$
C_{L}^{\mu e}=\sum_{A=1}^{9}\left[m_{\mu}\left[\hat{g}_{d_{L}}^{X}\right]_{A 1}^{*}\left[\hat{g}_{d_{L}}^{X}\right]_{A 2} F\left(\frac{m_{d_{A}}^{2}}{m_{X}^{2}}\right)+m_{d_{A}}\left[\hat{g}_{d_{L}}^{X}\right]_{A 1}^{*}\left[\hat{g}_{d_{R}}^{X}\right]_{A 2} G\left(\frac{m_{d_{A}}^{2}}{m_{X}^{2}}\right)\right] .
$$

$C_{R}^{\mu e}$ is obtained by formally replacing $L \leftrightarrow R$. The loop functions are defined as

$$
\begin{gathered}
F(t)=-\frac{4-16 t+39 t^{2}-28 t^{3}+t^{4}+6 t^{2}(1+2 t) \log t}{4(t-1)^{4}}, \\
G(t)=\frac{-4+27 t-24 t^{2}+t^{3}+6 t(1+2 t) \log t}{2(t-1)^{3}} .
\end{gathered}
$$

The second term will be dominant due to the chiral enhancement by the Yukawa coupling with Higgs bosons if $\delta_{d} \leq \mathcal{O}\left(v_{H}\right)$ in Eq. (2.24) is larger than the muon mass. Since the muon couples to one vectorlike down quark with $\mathcal{O}(1)$ mixing, the branching fraction is estimated as

$$
\mathrm{BR}(\mu \rightarrow e \gamma) \sim 1.2 \times 10^{-13} \times\left(\frac{5.0 \mathrm{TeV}}{m_{X}}\right)^{4}\left(\frac{g_{L}^{X} g_{R}^{X}}{0.005}\right)^{2}\left(\frac{\delta_{d}}{100 \mathrm{MeV}}\right)^{2}
$$

where $g_{L(R)}^{X}$ is a typical size of leptoquark couplings to mu/ electron in the left (right) current. Thus the Higgs boson couplings with the vectorlike down-type fermions should be so suppressed that the current limit $\operatorname{BR}(\mu \rightarrow e \gamma)<$ $4.2 \times 10^{-13}$ [101] is satisfied. This process can probe different parameter space from the $K_{L} \rightarrow \mu e$ decay and $\mu-e$ conversion which are induced at the tree level, since it directly constrains the leptoquark couplings to the SM charged leptons and vectorlike quarks. We will see that there are parameter spaces that can be probed only by $\mu \rightarrow e \gamma$ even if $\delta_{d}=10^{-4} v_{H}$ and the chiral enhancement effect is suppressed. In our numerical analysis, we will set $\delta_{u}=\delta_{d}=10^{-4} v_{H}$, so that the chiral enhancement effect to $\mu \rightarrow e \gamma$ is negligible and conservative limits are obtained.

Given the severe constraint from $\mu \rightarrow e \gamma$, the muon anomalous magnetic moment $\Delta a_{\mu} \sim \mathcal{O}\left(10^{-9}\right)$ is difficult to be explained by the one-loop effects of the leptoquark and vectorlike quark. It is roughly estimated as

$$
\begin{aligned}
\Delta a_{\mu} \lesssim & \frac{m_{\mu} \delta_{d}}{16 \pi^{2} m_{X}^{2}} N_{c} N_{\mathrm{VL}} \sim 5 \times 10^{-11} \\
& \times\left(\frac{5 \mathrm{TeV}}{m_{X}}\right)^{2}\left(\frac{\delta_{d}}{100 \mathrm{MeV}}\right)
\end{aligned}
$$




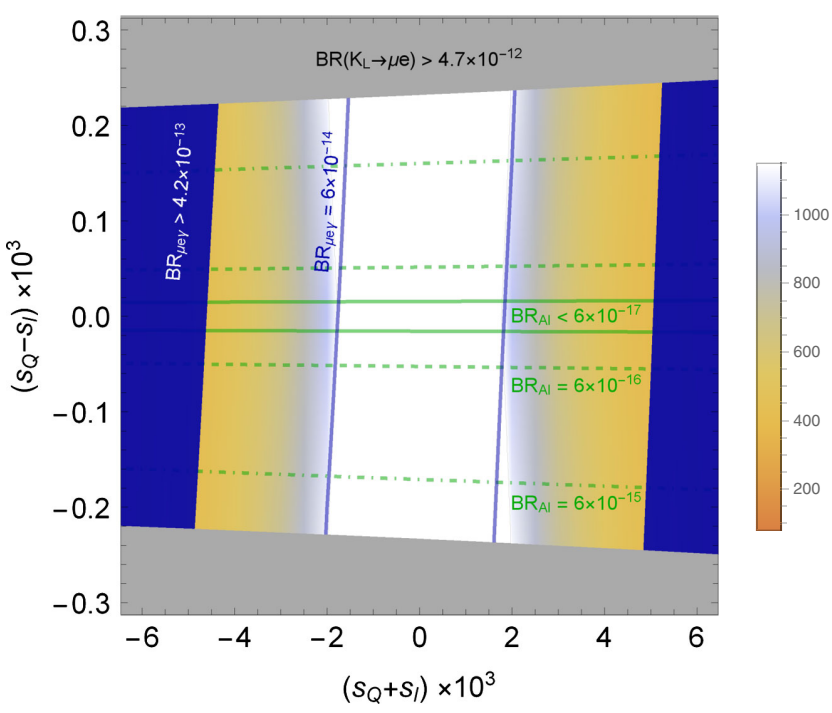

FIG. 1. The degree of tuning, the current constraints and future sensitivity in the $\left(s_{Q}+s_{\ell}, s_{Q}-s_{\ell}\right)$ plane, where $s_{Q}:=s_{Q_{L}}=$ $s_{Q_{R}}$ and $s_{\ell}:=s_{\ell_{L}}=s_{\ell_{R}}$. The density plot shows the degree of tuning $\Delta_{\mathrm{FT}}^{-1}$. The gray and blue shaded regions are excluded by the measurement of $\operatorname{BR}\left(K_{L} \rightarrow \mu e\right)$ and $\operatorname{BR}(\mu \rightarrow e \gamma)$, respectively. The blue and green lines show values of $\operatorname{BR}(\mu \rightarrow e \gamma)$ and $\mu-e$ conversion observables. See the text for further details.

where $N_{c}=3$ and $N_{\mathrm{VL}}=6$. Thus it is, at least, 2 orders of magnitude smaller than the currently preferred value as far as $\delta_{d} \lesssim m_{\mu}$ to suppress $\mu \rightarrow e \gamma{ }^{12}$

Figure 1 shows the degree of tuning $\Delta_{\mathrm{FT}}$ and constraints from the $\mu-e$ violations on the $\left(s_{Q}+s_{\ell}\right)$ vs $\left(s_{Q}-s_{\ell}\right)$ plane, where

$$
s_{Q}:=s_{Q_{L}}=s_{Q_{R}} \quad s_{\ell}:=s_{\ell_{L}}=s_{\ell_{R}} .
$$

The density plot shows $\Delta_{\mathrm{FT}}^{-1}$. The gray (blue) region is excluded by the current limit on $\operatorname{BR}\left(K_{L} \rightarrow \mu e\right)$ $[\operatorname{BR}(\mu \rightarrow e \gamma)]$. The blue lines are the future sensitivity of $\mathrm{BR}(\mu \rightarrow e \gamma)=6 \times 10^{-14}$ at the MEG experiment [102]. The green lines show $\operatorname{BR}(\mu \rightarrow e)^{\mathrm{Al}}$ and it is below the future sensitivity of $\operatorname{BR}(\mu \rightarrow e)^{\mathrm{Al}}=6 \times 10^{-17}[98]$ between the solid green lines. There is no region excluded by the current limit on $\operatorname{BR}(\mu \rightarrow e)^{\mathrm{Au}}$ [96]. Since the leptoquark couplings to the SM fermions are induced by $\left|s_{Q}-s_{\ell}\right|$, the tree-level $\mu-e$ violations are enhanced in the upper and lower regions. On the other hand, $\operatorname{BR}(\mu \rightarrow e \gamma)$ is enhanced as $\left|s_{Q}+s_{\ell}\right|$ increases since it comes from the coupling with the vectorlike quarks. The limit from

\footnotetext{
${ }^{12}$ It might be possible that $\Delta a_{\mu}$ is explained if $\delta_{d}$ for the two muons is $\mathcal{O}(10 \mathrm{GeV})$, while those which induce flavor violations are sufficiently suppressed. Such a situation could be realized by relaxing the relation of the angles in Eq. (3.10) and allowing flavor dependent mixing angles, although it may require another fine-tuning to sufficiently suppress $\mu \rightarrow e \gamma$ in addition to $K_{L} \rightarrow \mu e$.
}

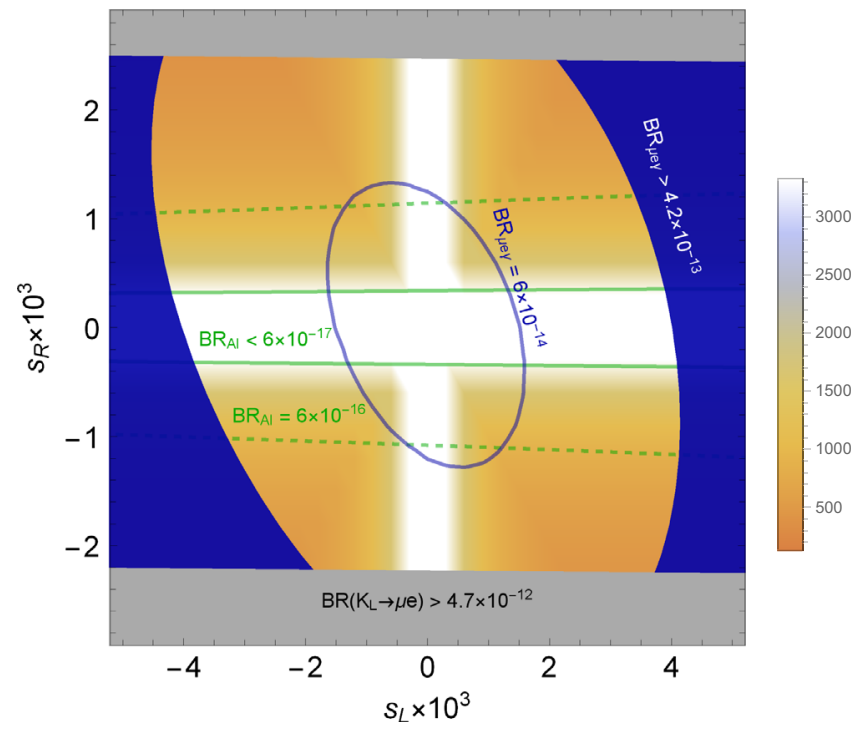

FIG. 2. The same plot as Fig. 1 on the $\left(s_{L}, s_{R}\right)$ plane where $s_{L}:=s_{Q_{L}}=-s_{\ell_{L}} / 1.1$ and $s_{R}:=s_{Q_{R}}=-s_{\ell_{R}} / 1.1$.

$\operatorname{BR}(\mu \rightarrow e \gamma)$ will be tightened if $\delta_{d}$ is larger. Note that the $b \rightarrow s \mu \mu$ anomaly is explained in the whole region in this plot.

Figure 1 indicates the degree of tuning $\Delta_{\mathrm{FT}}^{-1} \gtrsim 500$ (1000) at $\left|s_{Q}+s_{\ell}\right| \gtrsim 0.005$ (0.002). One may be concerned about the tuning between $s_{Q}$ and $s_{\ell}$, but the cancellation is mild since $\left(s_{Q}-s_{\ell}\right) /\left(s_{Q}+s_{\ell}\right) \gtrsim \mathcal{O}(0.01)$ even if $\operatorname{BR}(\mu \rightarrow e)^{\mathrm{Al}}<6 \times 10^{-17}$. Therefore, we conclude that $\Delta_{\mathrm{FT}}^{-1} \sim 500$ corresponding to $\mathcal{O}(0.2 \%)$ tuning is required to explain the $b \rightarrow s \mu \mu$ anomaly consistently with the current limit. Most of the parameter space, outside of the two green solid lines, will be tested by the future experiments of the $\mu-e$ conversion.

Figure 2 shows the same plot as Fig. 1, but on the $\left(s_{L}, s_{R}\right)$ plane, where

$s_{L}:=s_{Q_{L}}=-s_{\ell_{L}} / 1.1, \quad s_{R}:=s_{Q_{R}}=-s_{\ell_{R}} / 1.1$.

We keep $s_{Q_{L}} \sim s_{\ell_{L}}$ and $s_{Q_{R}} \sim s_{\ell_{R}}$ to avoid the large $\mu$-e violation. The tree-level $\mu$-e flavor violations are sensitive to $s_{R}$, since $\left[\hat{g}_{d_{L}}^{X}\right]_{s \mu}$ is sizable to explain the $b \rightarrow s \mu \mu$ anomaly. The future measurement of the $\mu \rightarrow e$ conversion will cover $\Delta_{\mathrm{FT}}^{-1} \lesssim 3000$ in this case. The radiative decay, $\mu \rightarrow e \gamma$ is equally sensitive to $s_{L}$ and $s_{R}$, and the region surrounded by the blue line will be covered by the MEG experiment.

\section{Flavor violation via Higgs bosons}

This model predicts sizable tree-level flavor violating couplings involving Higgs bosons even in the canonical limit. The heavy Higgs boson couplings to the down-type SM quarks are given by, in this limit, 


$$
\begin{aligned}
& {\left[\hat{Y}_{d}^{H}\right]_{i j} \sim-i\left[\hat{Y}_{d}^{A}\right]_{i j} \sim \frac{1}{\sqrt{2}}\left[\hat{Y}_{d}^{H^{+}}\right]_{i j}} \\
& \quad \sim-\frac{1}{\sqrt{2} v_{H}}\left(\frac{2 \tan \beta}{1-\tan ^{2} \beta}\left[D_{d}\right]_{i j}+\frac{1+\tan ^{2} \beta}{1-\tan ^{2} \beta}\left[V_{\mathrm{CKM}}^{\dagger} D_{u}\right]_{i j}\right),
\end{aligned}
$$

where $i, j=1,2,3$. Here we take the decoupling limit of the Higgs bosons. ${ }^{13}$ In this limit, all the scalar masses of the heavy Higgs doublet are degenerate. The second term in the parentheses inevitably induces flavor violations whose typical values are estimated as

$v_{H}^{-1}\left|V_{\mathrm{CKM}}^{\dagger} D_{u}\right| \sim\left(\begin{array}{ccc}5 \times 10^{-5} & 0.0006 & 0.007 \\ 1 \times 10^{-6} & 0.003 & 0.03 \\ 2 \times 10^{-8} & 0.0001 & 0.78\end{array}\right)$.

Flavor violating couplings, which are off-diagonal elements, are not small and hence large flavor violating

processes will be generated if the Higgs bosons are light, although the chirality structure may suppress them because only the upper-right elements are sizable.

Similarly, there are flavor violations in the charged lepton sector, which are estimated as $U_{\mathrm{PMNS}} D_{n} / v_{H}$. The effects are, however, expected to be negligible because the Yukawa coupling to the neutrinos is estimated as $D_{n} / v_{H} \sim \mathcal{O}\left(10^{-5}\right)$ to explain the neutrino masses with the $\mathcal{O}(10 \mathrm{TeV})$ Majorana masses. Therefore the flavor violation in the quark sector, especially the neutral meson mixing, is the most sensitive process to the effects of the extra Higgs bosons. The effective interactions relevant to our model are given by ${ }^{14}$

$$
\mathcal{H}_{\mathrm{eff}}^{\Delta F=2}=\sum_{I, A} C_{I}^{A} Q_{I}^{A}
$$

where $(I, A)=(1, \mathrm{VLL}),(2, \mathrm{LR}),(1, \mathrm{SLL}),(1, \mathrm{SRR})$. The four-Fermi operators are defined as

$$
\begin{aligned}
& Q_{1}^{\mathrm{VLL}}=\left(\bar{F}^{a} \gamma_{\mu} P_{L} f^{a}\right)\left(\bar{F}^{b} \gamma^{\mu} P_{L} f^{b}\right), \quad Q_{2}^{\mathrm{LR}}=\left(\bar{F}^{a} P_{L} f^{a}\right)\left(\bar{F}^{b} P_{R} f^{b}\right), \\
& Q_{1}^{\mathrm{SLL}}=\left(\bar{F}^{a} P_{L} f^{a}\right)\left(\bar{F}^{b} P_{L} f^{b}\right), \quad Q_{1}^{\mathrm{SRR}}=\left(\bar{F}^{a} P_{R} f^{a}\right)\left(\bar{F}^{b} P_{R} f^{b}\right),
\end{aligned}
$$

where $a, b=1,2,3$ are the color indices. Here, $(F, f)=(s, d),(b, d),(b, s)$ for $K-\bar{K}, B_{d}-\bar{B}_{d}$ and $B_{s}-\bar{B}_{s}$ mixing, respectively. We define the ratios of an off-diagonal matrix element of our model to that of the SM as

$$
C_{M}:=\frac{\left\langle M\left|\mathcal{H}_{\mathrm{eff}}^{\Delta F=2}\right| \bar{M}\right\rangle}{\left\langle M\left|\mathcal{H}_{\mathrm{eff}}^{\mathrm{SM}}\right| \bar{M}\right\rangle}=\frac{C_{\mathrm{SM}}^{M} \mathcal{O}_{1}^{V L L}+C_{2}^{L R} \mathcal{O}_{2}^{L R}+\left(C_{1}^{S L L}+C_{1}^{S R R}\right) \mathcal{O}_{1}^{S L L}}{C_{\mathrm{SM}}^{M} \mathcal{O}_{1}^{V L L}} .
$$

Here, $\mathcal{O}_{I}^{A}:=\left\langle M\left|Q_{I}^{A}\right| \bar{M}\right\rangle /\left(2 m_{M}\right)$ with $m_{M}$ being the meson mass. We use the values of $\mathcal{O}_{I}^{A}$ shown in Ref. [62]. The SM contribution $C_{\mathrm{SM}}^{M}$ is shown in e.g., Ref. [104]. In our model, the Wilson coefficients are given by

$$
C_{2}^{L R}=-\sum_{S=h, H, A} \frac{y_{L}^{S} y_{R}^{S}}{2 m_{S}^{2}}, \quad C_{1}^{S L L}=-\sum_{S=h, H, A} \frac{y_{L}^{S} y_{L}^{S}}{2 m_{S}^{2}}, \quad C_{1}^{S R R}=-\sum_{S=h, H, A} \frac{y_{R}^{S} y_{R}^{S}}{2 m_{S}^{2}},
$$

where

$$
y_{L}^{S}=\left[\hat{Y}_{d}^{S}\right]_{j i}^{*}, \quad y_{R}^{S}=\left[\hat{Y}_{d}^{S}\right]_{i j},
$$

with $(i, j)=(2,1),(3,1),(3,2)$ for $M=K, B_{d}, B_{s}$, respectively. The flavor violation from the adjoint field is negligible as discussed in Appendix A 3. We neglect loop corrections from the leptoquarks in our analysis. The current constraints at 95\% C.L. given by the UT-Fit $[105,106]$ are

$$
0.87 \leq \operatorname{Im} C_{K} \leq 1.39, \quad 0.83 \leq\left|C_{B_{d}}\right| \leq 1.29, \quad 0.942 \leq\left|C_{B_{s}}\right| \leq 1.288 .
$$

As pointed out in Ref. [107], the uncertainties are reduced in the ratio of mass differences of the $B_{d}$ and $B_{s}$ meson mixing,

$$
\frac{\Delta M_{d}}{\Delta M_{s}}=0.0298_{-0.0009}^{+0.0005}
$$

\footnotetext{
${ }^{13}$ See Appendix A 3 for more details of the Higgs couplings.

${ }^{14}$ We use the basis of the operators used in e.g., Ref. [103].
} 

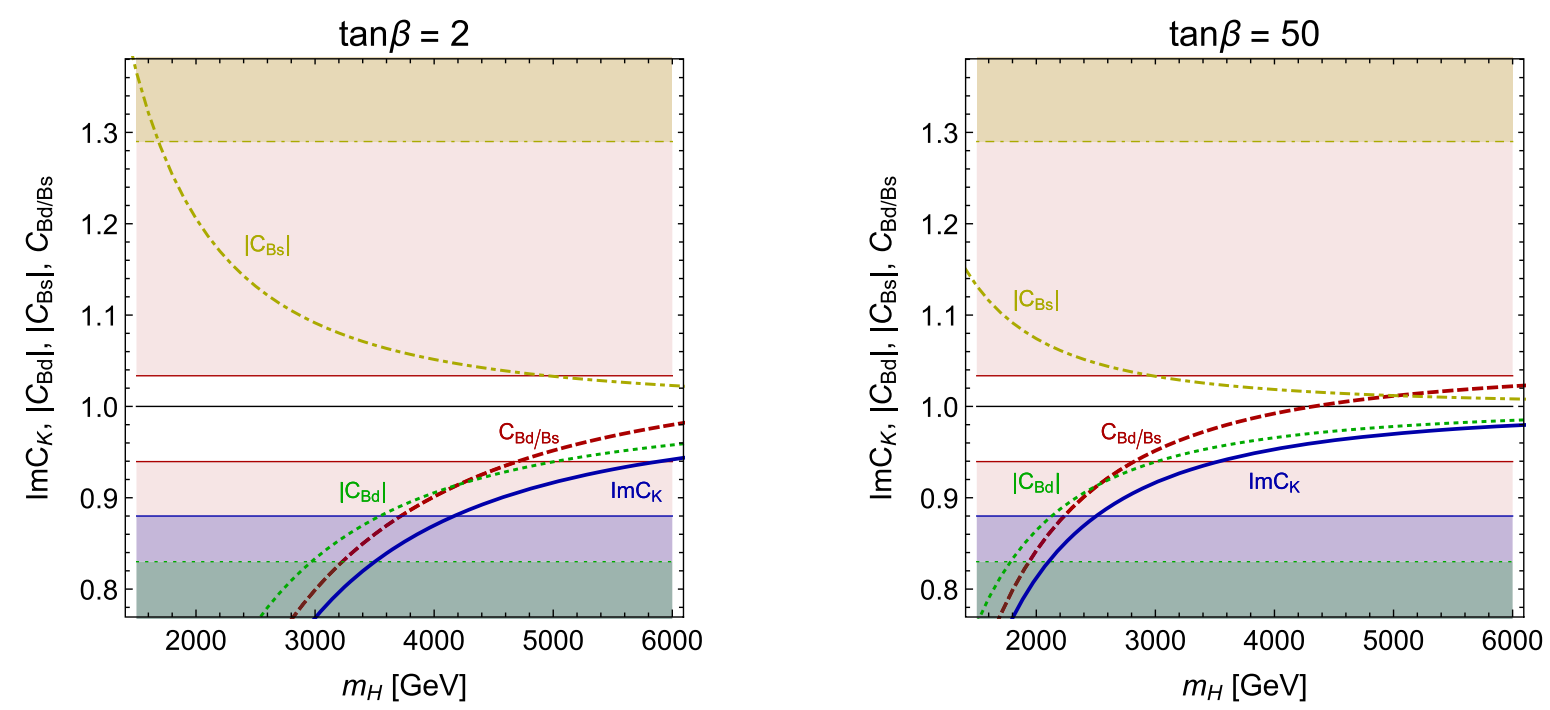

FIG. 3. The limits on the heavy Higgs boson mass $m_{H}$ from the observables concerned with the neutral meson mixings: $\left|C_{B_{d}}\right|$ (green), $\left|C_{B_{s}}\right|$ (yellow), $C_{B_{d} / B_{s}}$ (red) and $\operatorname{Im} C_{K}$ (blue). The excluded region for each observable is filled with the same color as the corresponding line. $\tan \beta$ is fixed at $\tan \beta=2.0$ (50) in the left (right) panel.

We define the parameter,

$$
C_{B_{d} / B_{s}}:=\frac{1}{0.0298} \times\left|\frac{\left\langle B_{d}\left|\mathcal{H}_{\mathrm{eff}}^{\Delta F=2}\right| \bar{B}_{d}\right\rangle}{\left\langle B_{s}\left|\mathcal{H}_{\mathrm{eff}}^{\Delta F=2}\right| \bar{B}_{s}\right\rangle}\right|
$$

Figure 3 shows experimental limits from the neutral meson mixing on the heavy Higgs boson mass for $\tan \beta=$ 2.0 (50) on the left (right) panel. Our predictions for $\left|C_{B_{d}}\right|$, $\left|C_{B_{s}}\right|, C_{B_{d} / B_{s}}$ and $\operatorname{Im} C_{K}$ are depicted by the green, yellow, red, and blue lines, respectively. The excluded region for each observable is filled with the same color as the corresponding line. In the red regions, the deviation of $C_{B_{d} / B_{s}}$ from the central value is more than twice as large as the uncertainties. In this figure, our parameters satisfy $s_{Q}+s_{\ell}=0.0015$ and $s_{Q}-s_{\ell}=0.6 \times 10^{-5}$ that cannot be probed by looking for the $\mu$-e flavor violations. The limits in the cases with larger angles $s_{Q}, s_{\ell}$ are quite similar to this result because the dominant effect comes from Eq. (3.22) which is independent of the angles, although there is mild dependence on them. We see that $C_{B_{d} / B_{s}}$ gives the most stringent limits for both $\tan \beta=2$ and 50. Interestingly, the bound from $C_{B_{d} / B_{s}}$ is stronger than the others, since the uncertainty is small and our predictions of $\left|C_{B_{s}}\right|$ and $\left|C_{B_{d}}\right|$ move in the opposite directions. The limits from the phases of $C_{B_{d, s}}$ are weaker than those from the absolute values. The lower bound on the heavy Higgs boson mass is about 4.8 (2.8) $\mathrm{TeV}$ for $\tan \beta=2.0$ (50). The limit is stronger for smaller $\tan \beta$ since the up-type Yukawa coupling constants are enhanced, see Eq. (3.22).

\section{C. $n-\bar{n}$ oscillation}

Before closing this section, let us discuss neutron antineutron $(n-\bar{n})$ oscillation. In general, gauge unified models predict baryon number violating processes, such as proton decays and $n-\bar{n}$ oscillation, which provide a useful tool to test the unification models. In the PS unification, the gauge bosons from the PS symmetry breaking do not mediate the baryon number violating processes, since their interactions respect the $B-L$ and $B+L$ symmetries. However, the scalars responsible for the PS symmetry breaking can generate the baryon number violation, depending on the representations. Since the PS breaking scale is relatively low in our model, the baryon number violation induced by such scalars may provide a stringent constraint.

In our model, three (nonsinglet) scalar fields, namely $\Phi:(\mathbf{1}, \mathbf{2}, \mathbf{2}), \Delta:(\mathbf{1 5}, \mathbf{1}, \mathbf{1})$ and $\Sigma:(\overline{\mathbf{1 0}}, \mathbf{1}, \mathbf{3})$, are introduced. With these representations, the stability of proton is ensured even after the PS symmetry breaking because of the discrete symmetry under the following transformation [108]:

$\Sigma_{a b} \rightarrow e^{-2 i \pi / 3} \Sigma_{a b}, \quad \Sigma_{a 1} \rightarrow e^{-i \pi / 3} \Sigma_{a 1}, \quad \Sigma_{11} \rightarrow \Sigma_{11}$,

$$
\mathbf{u}_{L, R} \rightarrow e^{i \pi / 3} \mathbf{u}_{L, R}, \quad \mathbf{d}_{L, R} \rightarrow e^{i \pi / 3} \mathbf{d}_{L, R},
$$

where $a, b=2,3,4$ correspond to the color indices. Since this symmetry does not protect processes that change the baryon number by an even integer, the $n-\bar{n}$ oscillation can occur in our model. In what follows, we shall estimate the transition amplitude and discuss the compatibility with the $\mathrm{TeV}$-scale leptoquark scenario.

Of three scalars in our model, only the symmetric representation scalar $\Sigma$ breaks the baryon number symmetry when it develops the VEV, and hence contributes to 
the $n-\bar{n}$ transition. The transition requires three $\Sigma \bar{f}_{R}^{c} h f_{R}$ vertices, the VEV of $\Sigma$ and the following quartic coupling of $\Sigma$ :

$$
V_{n-\bar{n}}=\lambda_{\Sigma}\left[\operatorname{Tr}\left(\Sigma_{\alpha \zeta} \Sigma_{\beta \eta}\right) \operatorname{Tr}\left(\Sigma_{\gamma \kappa} \Sigma_{\delta \lambda}\right) \epsilon^{\alpha \beta \gamma \delta} \epsilon^{\zeta \eta \kappa \lambda}+\text { H.c. }\right]
$$

where $\alpha, \beta, \ldots, \lambda=1,2,3,4$ are the $S U(4)_{C}$ indices and the trace is for the $S U(2)_{R}$ indices. After the PS symmetry breaking, the interactions relevant to the $n-\bar{n}$ oscillation are given by

$$
\begin{aligned}
V_{n-\bar{n}} \supset & 2 \lambda_{\Sigma} v_{\Sigma} \epsilon^{a b c} \epsilon^{d e f} \Sigma_{a d}^{2 / 3}\left(\Sigma_{b e}^{-1 / 3} \Sigma_{c f}^{-1 / 3}+2 \Sigma_{b e}^{2 / 3} \Sigma_{c f}^{-4 / 3}\right) \\
& + \text { H.c., }
\end{aligned}
$$

where $a, b, \ldots, f=1,2,3$ are the $S U(3)_{c}$ indices and we denote the scalars of symmetric representation under $S U(3)_{c}$ as $\Sigma^{Y}:(\overline{\mathbf{6}}, \mathbf{1}, Y)$, with $Y=-4 / 3,-1 / 3,2 / 3$, under $G_{\mathrm{SM}}$. Their masses are denoted by $m_{\Sigma^{Y}}$. Integrating out these heavy scalars induces six-quark operators,

$$
\begin{aligned}
\mathcal{L}_{\mathrm{eff}}^{n-\bar{n}}= & -\lambda_{\Sigma} v_{\Sigma} \epsilon^{a b c} \epsilon^{d e f} \\
& \times\left[\frac{h_{d d}^{2} h_{u u}}{m_{\Sigma^{-4 / 3}}^{4} m_{\Sigma^{2 / 3}}^{2}}\left(\bar{d}_{R, a}^{c} d_{R, d}\right)\left(\bar{d}_{R, b}^{c} d_{R, e}\right)\left(\bar{u}_{R, c}^{c} u_{R, f}\right)\right. \\
& \left.-2 \frac{h_{d d} h_{u d}^{2}}{m_{\Sigma^{-4 / 3}}^{2} m_{\Sigma^{-1 / 3}}^{4}}\left(\bar{d}_{R, a}^{c} d_{R, d}\right)\left(\bar{d}_{R, b}^{c} u_{R, e}\right)\left(\bar{u}_{R, c}^{c} d_{R, f}\right)\right],
\end{aligned}
$$

where $h_{q q^{\prime}}\left(q, q^{\prime}=u, d\right)$ is the Yukawa coupling of $\Sigma$ to $q$ and $q^{\prime}$ quarks in the mass basis, given by

$$
h_{q q^{\prime}}=\sum_{i, j=1,2,3} \frac{1}{\sqrt{2}}[h]_{i j}\left[U_{R}^{q}\right]_{3+i, 1}\left[U_{R}^{q^{\prime}}\right]_{3+j, 1} .
$$

Here let us assume for simplicity

$$
m_{\Sigma}:=m_{\Sigma^{-4 / 3}}=m_{\Sigma^{2 / 3}}=m_{\Sigma^{-1 / 3}}, \quad h_{Q}:=h_{u u}=h_{d d}=h_{u d} .
$$

The Yukawa coupling of the SM quarks to $\Sigma$ is estimated to be $h_{Q} \sim s_{Q}^{2}$ when the Yukawa coupling in the gauge basis $h \sim \mathcal{O}(1)$, and hence it is suppressed by the mixing between the chiral and vectorlike quarks which is at most 0.005 as read from Fig. 1. With the naive dimensional analysis [109], we find the transition amplitude to be

$$
\begin{aligned}
\tau_{n-\bar{n}} \sim & \frac{m_{\Sigma}^{6}}{\lambda_{\Sigma} v_{\Sigma} h_{Q}^{3}} \frac{1}{\Lambda_{\mathrm{QCD}}^{6}} \\
\sim & 1.2 \times 10^{14} \mathrm{sec} \times\left(\frac{m_{\Sigma}}{10 \mathrm{TeV}}\right)^{6}\left(\frac{10 \mathrm{TeV}}{v_{\Sigma}}\right) \\
& \times\left(\frac{180 \mathrm{MeV}}{\Lambda_{\mathrm{QCD}}}\right)^{6}\left(\frac{0.005}{s_{Q}}\right)^{6}\left(\frac{1.0}{h}\right)^{3}\left(\frac{0.1}{\lambda_{\Sigma}}\right) .
\end{aligned}
$$

Here, $\Lambda_{\mathrm{QCD}}$ denotes the QCD scale. It follows that the $n-\bar{n}$ transition is very sensitive to the $\Sigma$ mass. The current limit is $[110,109]$

$\tau_{n-\bar{n}} \geq\left\{\begin{array}{lll}2.7 \times 10^{8} & \text { sec } & \text { bound neutron } \\ 8.6 \times 10^{7} & \text { sec } & \text { reactor free neutron } .\end{array}\right.$

Therefore, the constraint from the $n-\bar{n}$ oscillation can be avoided even if the relevant couplings, $h$ and $\lambda_{\Sigma}$, are of order unity.

\section{SUMMARY}

In this paper, we have proposed an explicit model with the PS gauge symmetry and extra vectorlike fermions that realizes

(i) a vector leptoquark which accounts for the $b \rightarrow s \mu \mu$ anomaly

(ii) the realistic mass spectrum of the SM fermions

(iii) sufficiently suppressed $\mu$ - $e$ flavor violations due to tuning of the parameters.

The texture in Eq. (2.23) of the vectorlike fermion masses is a key idea to suppress the $\mu$ - $e$ flavor violation, especially $K_{L} \rightarrow \mu e$ decay. We have shown in Fig. 1 that $\operatorname{BR}\left(K_{L} \rightarrow e \mu\right)$ is less than the current limit if we allow for the $\mathcal{O}(0.2 \%)$ tuning of the parameters. With this texture, the $\mathrm{TeV}$-scale vector leptoquark is allowed and thus the $b \rightarrow s \mu \mu$ anomaly can be explained. We have, on the other hand, pointed out that the combination of the $Z^{\prime}$ boson and vectorlike quark searches exclude the light enough leptoquark to explain the $b \rightarrow c \tau \nu$ anomaly.

The idea to relax the bound from $K_{L} \rightarrow \mu e$ by introducing vectorlike families was proposed in Ref. [21]. In this paper, we have constructed a more economical setup and have shown an explicit texture of the mass matrices to suppress the $K_{L} \rightarrow \mu e$ as well as explaining the SM fermion mass and mixing matrices. By this explicit construction, we found that the $b \rightarrow c \tau \nu$ anomaly is difficult to be explained, the $\mathcal{O}(0.1) \%$ tuning is required to satisfy the phenomenological conditions for the $\mathcal{O}(1) \mathrm{TeV}$ leptoquark, and the flavor violating couplings of the heavy Higgs boson are unavoidable.

On top of these, we conclude that the available parameter space to explain the $b \rightarrow s \mu \mu$ anomaly is probed by future searches for $\mu \rightarrow e \gamma$ and $\mu \mathrm{Al} \rightarrow e \mathrm{Al}$ processes. The former process is sensitive to the couplings with the SM leptons and vectorlike quarks, while the latter is sensitive to the SM 
leptons and SM down quarks. The future experiments cover the parameter space that satisfies $\left|s_{Q}+s_{\ell}\right| \gtrsim 0.002$ and $\left|s_{Q}-s_{\ell}\right| \gtrsim 3.0 \times 10^{-5}$. We have also found that the flavor violating couplings of the extra Higgs bosons are inevitable. It is shown in Fig. 3 that the heavy Higgs bosons lighter than 4.8 (2.8) $\mathrm{TeV}$ are excluded by the measurement of $B$ meson mixing when $\tan \beta=2$ (50). In our model, the neutron oscillation is also predicted by the couplings involving $\Sigma$. We estimate the transition amplitude and conclude that our prediction is much below the current experimental bound.

Let us outline how to realize the hierarchy of Eq. (2.22) required to suppress $K_{L} \rightarrow \mu e$ with the TeV-scale leptoquark and possible UV completion of the model. There should be $\mathcal{O}(0.1 \%)$ cancellation between the vectorlike masses and masses from the Yukawa couplings with the adjoint field $\Delta$. The vectorlike masses may need to be replaced by a field with nonzero VEV, and the VEV of the field should be related to that of the adjoint field. Such a relation between two VEVs, for instance, could arise if the model is extended by supersymmetry, so that quartic couplings are determined by group factors and gauge couplings. Further, the cancellation would be realized if the Yukawa couplings have a certain structure constrained by flavor symmetries. Our PS model could be embedded into a larger gauge group such as $S O(10)$ symmetry. In that case, the coupling constants should be perturbative up to a scale where the UV theory appears. The gauge coupling constants are perturbative up to the typical GUT scale with the matter contents of our model, but the Landau pole appears at a lower scale if there are more vectorlike fermions. Thus the matter contents will be more constrained if we consider the GUT models. The study for such UV completion as well as analysis with a concrete scalar potential are interesting and important to explain the hierarchy of Eq. (2.22), but this is our future work.

Before closing our discussion, let us comment on loop corrections to the flavor violating processes, e.g., $K_{L} \rightarrow \mu e$. In our setup, the tree-level contributions via $\Delta$ and leptoquark exchanging are very suppressed because of the unique structure in the fermion mass matrices. This setup leads almost flavor-diagonal couplings, and we concentrate mainly on the tree-level predictions induced by leptoquark exchanging. However, the leptoquark and scalar couplings to quarks/leptons and heavy fermions could be sizable. The one-loop corrections involving heavy fermions and scalars may be large in the TeV-scale scenario. The study on the loop effect is not so simple because of many parameters. We need more careful study, taking into account the mass spectrum of all fermions as well. This study is work in progress and will be shown in the near future.

\section{ACKNOWLEDGMENTS}

S. I. would like to thank the warm hospitality at KEK where he stayed during the work. The work of S. I. is supported by the Japan Society for the Promotion of Science (JSPS) Research Fellowships for Young Scientists, No. 19J10980, and the JSPS Core-to-Core Program, No. JPJSCCA20200002. The work of J. K. is supported in part by the Institute for Basic Science (IBSR018-D1), the Department of Energy (DOE) under Award No. DE-SC0011726, and the Grant-in-Aid for Scientific Research from the Ministry of Education, Science, Sports and Culture (MEXT), Japan No. 18K13534. The work of S. O. is supported in part by NSERC, Canada. The work of Y. O. is supported by Grant-in-Aid for Scientific research from the MEXT, Japan, No. 19H04614, No. 19H05101, and No. 19K03867.

Note added.-Recently, the LHCb collaboration reported the new result on the lepton flavor universality in $B \rightarrow K \ell \ell$ with full run 2 data on the arXiv [111]. The result is consistent with the previous result used in our analysis and does not change our discussion drastically.

\section{APPENDIX A: MODEL DETAILS}

\section{Diagonalization of the fermion mass matrices \\ a. Dirac mass matrix}

Let us discuss the fermion mass matrices parametrized as in Eq. (2.24). The unitary matrices

$U_{\ell_{L}}^{0}=\left(\begin{array}{cc}0_{6 \times 3} & V_{\ell_{L}} \\ W_{\ell_{L}} & 0_{3 \times 6}\end{array}\right), \quad U_{\ell_{R}}^{0}=\left(\begin{array}{cc}V_{\ell_{R}} & 0_{6 \times 3} \\ 0_{3 \times 6} & W_{\ell_{R}}\end{array}\right), \quad U_{Q_{L}}^{0}=\left(\begin{array}{cc}V_{Q_{L}} & 0_{6 \times 3} \\ 0_{3 \times 6} & W_{Q_{L}}\end{array}\right), \quad U_{Q_{R}}^{0}=\left(\begin{array}{cc}0_{6 \times 3} & V_{Q_{R}} \\ W_{Q_{R}} & 0_{3 \times 6}\end{array}\right)$,

approximately diagonalize the mass matrices as

$$
\left(U_{\ell_{L}}^{0}\right)^{\dagger} \mathcal{M}_{e} U_{\ell_{R}}^{0}=\left(\begin{array}{ccc}
D_{\ell_{R}} & 0_{3} & \cdot \\
\cdot & d_{e} & 0_{3} \\
\cdot & \cdot & D_{\ell_{L}}
\end{array}\right), \quad\left(U_{Q_{L}}^{0}\right)^{\dagger} \mathcal{M}_{d} U_{Q_{R}}^{0}=\left(\begin{array}{ccc}
D_{Q_{L}} & \cdot & \cdot \\
0_{3} & d_{d} & \cdot \\
\cdot & 0_{3} & D_{Q_{R}}
\end{array}\right),
$$




$$
\left(U_{\ell_{L}}^{0}\right)^{\dagger} \mathcal{M}_{n} U_{\ell_{R}}^{0}=\left(\begin{array}{ccc}
D_{\ell_{R}} & 0_{3} & \cdot \\
\cdot & m_{n} & 0_{3} \\
\cdot & \cdot & D_{\ell_{L}}
\end{array}\right), \quad\left(U_{Q_{L}}^{0}\right)^{\dagger} \mathcal{M}_{u} U_{Q_{R}}^{0}=\left(\begin{array}{ccc}
D_{Q_{L}} & \cdot & \cdot \\
0_{3} & m_{u} & \cdot \\
\cdot & 0_{3} & D_{Q_{R}}
\end{array}\right),
$$

where represents $\mathcal{O}\left(v_{H}\right)$ entries. Here, $d_{e}$ and $d_{d}$ are chosen to be diagonal by using the redundancies of Eq. (2.27) in $V_{\ell_{L . R}}$ and $V_{Q_{L, R}}$. To make the singular values in increasing order and the SM up-type quark and neutrino masses, $M_{u}$ and $M_{n}$, diagonalized, we introduce

$$
\begin{aligned}
& \tilde{U}_{e_{L, R}}:=U_{\ell_{L, R}}^{0}\left(\begin{array}{ccc}
0_{3} & \mathbf{1}_{3} & 0_{3} \\
\mathbf{1}_{3} & 0_{3} & 0_{3} \\
0_{3} & 0_{3} & \mathbf{1}_{3}
\end{array}\right), \quad \tilde{U}_{n_{L, R}}:=U_{\ell_{L, R}}^{0}\left(\begin{array}{ccc}
0_{3} & \mathbf{1}_{3} & 0_{3} \\
u_{n_{L, R}} & 0_{3} & 0_{3} \\
0_{3} & 0_{3} & \mathbf{1}_{3}
\end{array}\right), \\
& \tilde{U}_{d_{L, R}}:=U_{Q_{L, R}}^{0}\left(\begin{array}{lll}
0_{3} & \mathbf{1}_{3} & 0_{3} \\
\mathbf{1}_{3} & 0_{3} & 0_{3} \\
0_{3} & 0_{3} & \mathbf{1}_{3}
\end{array}\right), \quad \tilde{U}_{u_{L, R}}:=U_{Q_{L, R}}^{0}\left(\begin{array}{ccc}
0_{3} & \mathbf{1}_{3} & 0_{3} \\
u_{u_{L, R}} & 0_{3} & 0_{3} \\
0_{3} & 0_{3} & \mathbf{1}_{3}
\end{array}\right),
\end{aligned}
$$

where the unitary matrices $u_{u_{L, R}}$ and $u_{n_{L, R}}$ diagonalize $m_{u}$ and $m_{n}$ respectively,

$$
u_{n_{L}}^{\dagger} m_{n} u_{n_{R}}=d_{n}, \quad u_{u_{L}}^{\dagger} m_{u} u_{u_{R}}=d_{u} .
$$

The Dirac mass matrices are diagonalized as

$$
\begin{array}{ll}
\left(\tilde{U}_{e_{L}}\right)^{\dagger} \mathcal{M}_{e} \tilde{U}_{e_{R}} \sim \operatorname{diag}\left(d_{e}, D_{\ell_{R}}, D_{\ell_{L}}\right), & \left(\tilde{U}_{n_{L}}\right)^{\dagger} \mathcal{M}_{n} \tilde{U}_{n_{R}} \sim \operatorname{diag}\left(d_{n}, D_{\ell_{R}}, D_{\ell_{L}}\right), \\
\left(\tilde{U}_{d_{L}}\right)^{\dagger} \mathcal{M}_{d} \tilde{U}_{d_{R}} \sim \operatorname{diag}\left(d_{d}, D_{Q_{R}}, D_{Q_{L}}\right), & \left(\tilde{U}_{u_{L}}\right)^{\dagger} \mathcal{M}_{u} \tilde{U}_{u_{R}} \sim \operatorname{diag}\left(d_{u}, D_{Q_{R}}, D_{Q_{L}}\right) .
\end{array}
$$

The corrections from the off-diagonal blocks to the SM fermion mass matrix are $\mathcal{O}\left(d_{f}^{2} / v_{\Delta}\right), f=e, n, d, u$, so it may be subdominant compared with the leading matrix $\sim d_{f}$.

\section{b. Neutrino masses}

The $18 \times 18$ mass matrix is given by

$-\mathcal{L}_{N}=\frac{1}{2} \overline{\mathbf{N}}_{L} \mathcal{M}_{N} \mathbf{N}_{R}=\left(\begin{array}{ll}\overline{\mathbf{n}}_{L} & \overline{\mathbf{n}}_{R}^{c}\end{array}\right)\left(\begin{array}{cc}0 & \mathcal{M}_{n} \\ \mathcal{M}_{n}^{T} & \mathcal{M}_{R}\end{array}\right)\left(\begin{array}{l}\mathbf{n}_{L}^{c} \\ \mathbf{n}_{R}\end{array}\right)$,

where the Dirac mass matrix $\mathcal{M}_{n}$ and Majorana mass matrix $\mathcal{M}_{n}$ are given in Eqs. (2.24) and (2.14), respectively. The mass basis is defined as

$$
\hat{\mathbf{N}}_{R}=U_{N}^{\dagger} \mathbf{N}_{R}, \quad \hat{\mathbf{N}}_{L}=U_{N}^{T} \mathbf{N}_{L},
$$

where $U_{N}^{T} \mathcal{M}_{N} U_{N}$ is diagonalized.

We introduce the unitary matrices,

$$
\tilde{U}_{N}=\left(\begin{array}{cc}
\tilde{U}_{n_{L}}^{*} & 0_{9} \\
0_{9} & \tilde{U}_{n_{R}}
\end{array}\right)
$$

where $\tilde{U}_{n_{L, R}}$ are defined in Eq. (A4). After multiplying these matrices, we obtain

$$
\begin{aligned}
\widetilde{\mathcal{M}}_{N}: & =\tilde{U}_{N}^{T} \mathcal{M}_{N} \tilde{U}_{N} \\
& =\left(\begin{array}{cccccc}
0_{3} & 0_{3} & 0_{3} & d_{n} & \cdot & 0_{3} \\
0_{3} & 0_{3} & 0_{3} & 0_{3} & D_{n_{R}} & \cdot \\
0_{3} & 0_{3} & 0_{3} & \cdot & \cdot & D_{n_{L}} \\
d_{n} & 0_{3} & \cdot & \tilde{M}_{R} & \tilde{\mu}_{N} & 0_{3} \\
\cdot & D_{n_{R}} & \cdot & \tilde{\mu}_{N}^{T} & \tilde{M}_{N} & 0_{3} \\
0_{3} & \cdot & D_{n_{L}} & 0_{3} & 0_{3} & 0_{3}
\end{array}\right),
\end{aligned}
$$

where

$$
\begin{aligned}
\left(\begin{array}{cc}
\tilde{M}_{R} & \tilde{\mu}_{N} \\
\tilde{\mu}_{N}^{T} & \tilde{M}_{N}
\end{array}\right)= & \left(\begin{array}{cc}
0_{3} & u_{n_{R}}^{T} \\
\mathbf{1}_{3} & 0_{3}
\end{array}\right) V_{\ell_{R}}^{T}\left(\begin{array}{cc}
M_{R} & 0_{3} \\
0_{3} & 0_{3}
\end{array}\right) \\
& \times V_{\ell_{R}}\left(\begin{array}{cc}
0_{3} & \mathbf{1}_{3} \\
u_{n_{R}} & 0_{3} .
\end{array}\right) .
\end{aligned}
$$

In numerical analysis, we studied the case in which $V_{\ell_{R}} \sim \mathbf{1}_{6}, u_{n_{R}} \sim \mathbf{1}_{3}$ and $M_{R} \propto \mathbf{1}_{3}$. We assume this structure in the analytical analysis in this Appendix, but the nonzero effects are included in our numerical analysis. The SM Majorana neutrino masses arise after the block diagonalization by 


$$
\tilde{U}_{N}^{1} \sim\left(\begin{array}{cccccc}
\mathbf{1}_{3} & 0_{3} & 0_{3} & d_{n} \tilde{M}_{R}^{-1} & 0_{3} & 0_{3} \\
0_{3} & \mathbf{1}_{3} & 0_{3} & 0_{3} & 0_{3} & 0_{3} \\
0_{3} & 0_{3} & \mathbf{1}_{3} & 0_{3} & 0_{3} & 0_{3} \\
-\tilde{M}_{R}^{-1} d_{n} & 0_{3} & 0_{3} & \mathbf{1}_{3} & 0_{3} & 0_{3} \\
0_{3} & 0_{3} & 0_{3} & 0_{3} & \mathbf{1}_{3} & 0_{3} \\
0_{3} & 0_{3} & 0_{3} & 0_{3} & 0_{3} & \mathbf{1}_{3}
\end{array}\right) .
$$

The other states have diagonal Dirac mass matrices. In this case, the active neutrino mass is approximately given by

$$
\tilde{M}_{\nu} \sim-d_{n} \tilde{M}_{R}^{-1} d_{n}
$$

and this is already diagonalized. The total unitary matrix is thus given by $U_{N} \sim \tilde{U}_{N} \tilde{U}_{N}^{1}$.

\section{Gauge interactions}

We shall discuss gauge interactions. The PS symmetry is broken by nonzero VEVs of $\Delta$ and $\Sigma$. We name the massive gauge bosons in $S U(4)_{C} /\left[S U(3)_{C} \times U(1)_{B-L}\right]$ as leptoquark $X_{\mu}$, and those in $\left[S U(2)_{R} \times U(1)_{B-L}\right] / U(1)_{Y}$ as $Z_{\mu}^{\prime}$ and $W_{R \mu}^{ \pm}$. In this subsection for the gauge interactions, $A, B, \ldots=1,2, \ldots, 15$ and $\alpha, \beta, \ldots=1,2,3,4$ are for the $S U(4)_{C}$ indices of adjoint and fundamental representations, respectively. The $S U(2)_{X}, X=L, R$, indices of adjoint and fundamental representations are respectively denoted as $k_{X}, \ldots=1,2,3$ and $\alpha_{X}, \ldots=1,2,3$.

\section{a. Symmetry breaking and vector boson masses}

The covariant derivative terms of the symmetry breaking scalar fields are given by

$$
\begin{aligned}
\mathcal{L}_{\text {kin }}^{\mathrm{SC}}= & \operatorname{Tr}_{4}\left(D^{\mu} \Delta \cdot D_{\mu} \Delta\right)+\sum_{\alpha, \beta} \operatorname{Tr}_{2}\left[\left(D^{\mu} \Sigma_{\alpha \beta}\right)^{\dagger} D_{\mu} \Sigma_{\alpha \beta}\right] \\
& +\operatorname{Tr}_{2}\left[\left(D^{\mu} \Phi\right)^{\dagger} D_{\mu} \Phi\right]
\end{aligned}
$$

where $\operatorname{Tr}_{4}$ is the trace for the $S U(4)_{C}$ indices and $\operatorname{Tr}_{2}$ is that for the $S U(2)_{L}$ and $S U(2)_{R}$ indices. The covariant derivatives are given by

$$
\begin{gathered}
D_{\mu} \Delta=\partial_{\mu} \Delta-i g_{4}\left[\mathcal{V}_{\mu}, \Delta\right], \\
D_{\mu} \Sigma_{\alpha \beta}=\partial_{\mu} \Sigma_{\alpha \beta}-i g_{R}\left[W_{R \mu}, \Sigma_{\alpha \beta}\right]+i g_{4} \Sigma_{\gamma \delta}\left(\mathcal{V}_{\alpha \mu}^{\gamma} \delta_{\beta}^{\delta}+\mathcal{V}_{\beta \mu}^{\delta} \delta_{\alpha}^{\gamma}\right), \\
D_{\mu} \Phi=\partial_{\mu} \Phi-i g_{L} W_{L \mu} \Phi+i g_{R} \Phi W_{R \mu},
\end{gathered}
$$

where the indices of the fields are

$$
\begin{gathered}
\Delta=\Delta_{\beta}^{\alpha}=\Delta^{A} T^{A}, \quad \Sigma_{\alpha \beta}=\sqrt{1+\delta_{\alpha \beta}} \Sigma_{\alpha \beta}^{k_{R}} \tau_{R}^{k_{R}}, \quad \Phi=\left(\begin{array}{ll}
H_{1} & \tilde{H}_{2}
\end{array}\right), \\
\mathcal{V}_{\mu}=\mathcal{V}_{\mu}^{A} T^{A}, \quad W_{L \mu}=W_{L \mu}^{k_{L}} \tau_{L}^{k_{L}}, \quad W_{R \mu}=W_{R \mu}^{k_{R}} \tau_{R}^{k_{R}},
\end{gathered}
$$

where $\Sigma^{\alpha \beta}=\Sigma^{\beta \alpha}$. Here, the scalar fields $\Delta^{A}$ and $\Sigma_{\alpha \beta}^{k_{R}}$ are canonically normalized. The Hermitian matrices $T^{A}$ and $\tau_{L(R)}^{k_{L(R)}}$ are the generators of fundamental representation of $S U(4)_{C}$ and $S U(2)_{L(R)}$, respectively. $H_{1}$ and $\tilde{H}_{2}:=\epsilon H_{2}^{*}$ are the $S U(2)_{L}$ doublets.

In this paper, we assume a certain potential gives the following VEVs:

$$
\langle\Delta\rangle=\frac{v_{\Delta}}{2 \sqrt{3}}\left(\begin{array}{cc}
3 & 0 \\
0 & -\mathbf{1}_{3}
\end{array}\right), \quad\left\langle\Sigma_{\alpha \beta}\right\rangle=\frac{v_{\Sigma}}{\sqrt{2}}\left(\begin{array}{cc}
0 & 1 \\
0 & 0
\end{array}\right) \otimes \delta_{\alpha 1} \delta_{\beta 1}, \quad\langle\Phi\rangle=v_{H}\left(\begin{array}{cc}
c_{\beta} & 0 \\
0 & s_{\beta}
\end{array}\right),
$$

where $s_{\beta}^{2}+c_{\beta}^{2}=1$. The $S U(4)_{C}$ gauge boson $\mathcal{V}_{\mu}$ and $S U(2)_{L, R}$ gauge boson $\mathcal{W}_{L, R \mu}$ are decomposed as

$$
\begin{aligned}
\mathcal{V} & =\sqrt{\frac{3}{8}}\left(\begin{array}{cc}
1 & 0 \\
0 & -\frac{1}{3} \cdot \mathbf{1}_{3}
\end{array}\right) V_{\mu}^{B-L}+\left(\begin{array}{cc}
0 & X^{\dagger} / \sqrt{2} \\
X / \sqrt{2} & G
\end{array}\right), \\
W_{L, R} & =\frac{1}{2}\left(\begin{array}{cc}
W_{L, R}^{3} & \sqrt{2} W_{L, R}^{+} \\
\sqrt{2} W_{L, R}^{-} & -W_{L, R}^{3}
\end{array}\right), \quad W_{L, R}^{ \pm}=\frac{W_{L, R}^{1} \pm i W_{L, R}^{2}}{\sqrt{2}},
\end{aligned}
$$

where the Lorentz index is omitted. Here, $V^{B-L}, X, G$ are $B-L$ gauge boson, leptoquarks and gluon, respectively. The mass terms of the gauge bosons are given by

$$
\mathcal{L}_{\text {mass }}^{V}=m_{X}^{2} X_{\mu}^{\dagger} X^{\mu}+\mathcal{W}_{\mu}^{+} \mathcal{M}_{W}^{2} \mathcal{W}^{-\mu}+\frac{1}{2} \mathcal{Z}_{\mu} \mathcal{M}_{Z}^{2} \mathcal{Z}^{\mu}
$$


where the mass and matrices are given by

$$
\begin{gathered}
m_{X}=g_{4} \sqrt{\frac{4}{3} v_{\Delta}^{2}+\frac{1}{2} v_{\Sigma}^{2}}, \quad \mathcal{M}_{W}^{2}=\frac{1}{2}\left(\begin{array}{cc}
g_{L}^{2} v_{H}^{2} & -s_{2 \beta} g_{L} g_{R} v_{H}^{2} \\
-s_{2 \beta} g_{L} g_{R} v_{H}^{2} & g_{R}^{2}\left(v_{H}^{2}+v_{\Sigma}^{2}\right)
\end{array}\right), \\
\mathcal{M}_{Z}^{2}=\frac{1}{2}\left(\begin{array}{ccc}
g_{L}^{2} v_{H}^{2} & -g_{L} g_{R} v_{H}^{2} & 0 \\
-g_{L} g_{R} v_{H}^{2} & g_{R}^{2} v_{H}^{2}+2 g_{R}^{2} v_{\Sigma}^{2} & -\sqrt{6} g_{R} g_{4} v_{\Sigma}^{2} \\
0 & -\sqrt{6} g_{R} g_{4} v_{\Sigma}^{2} & 3 g_{4}^{2} v_{\Sigma}^{2}
\end{array}\right),
\end{gathered}
$$

with the gauge bosons

$$
\mathcal{W}_{\mu}^{ \pm}:=\left(\begin{array}{c}
W_{L \mu}^{ \pm} \\
W_{R \mu}^{ \pm}
\end{array}\right), \quad \mathcal{Z}_{\mu}:=\left(\begin{array}{c}
W_{L \mu}^{3} \\
W_{R \mu}^{3} \\
V_{\mu}^{B-L}
\end{array}\right) .
$$

Here, $s_{2 \beta}=2 c_{\beta} s_{\beta}$ and $c_{2 \beta}=c_{\beta}^{2}-s_{\beta}^{2}$. The mass basis of the gauge bosons is defined as

$$
\mathcal{W}_{\mu}^{ \pm}=R_{W}\left(\begin{array}{c}
W_{\mu}^{ \pm} \\
W_{\mu}^{\prime \pm}
\end{array}\right), \quad \mathcal{Z}_{\mu}=R_{Z}\left(\begin{array}{c}
A_{\mu} \\
Z_{\mu} \\
Z_{\mu}^{\prime}
\end{array}\right)
$$

where $R_{W}$ and $R_{Z}$ are orthogonal matrices diagonalizing $\mathcal{M}_{W}^{2}$ and $\mathcal{M}_{Z}^{2}$, respectively.

The rotation matrix $R_{W}$ and eigenvalues of the mass matrix, $m_{W}$ and $m_{W^{\prime}}$ are exactly given by

$$
\begin{gathered}
R_{W}=\left(\begin{array}{cc}
c_{\omega} & s_{\omega} \\
-s_{\omega} & c_{\omega}
\end{array}\right), \\
m_{W}^{2}=\frac{v_{\Sigma}^{2}}{4}\left[g_{R}^{2}+\left(g_{R}^{2}+g_{L}^{2}\right) \eta-D_{W}\right], \quad m_{W^{\prime}}^{2}=\frac{v_{\Sigma}^{2}}{4}\left[g_{R}^{2}+\left(g_{R}^{2}+g_{L}^{2}\right) \eta+D_{W}\right],
\end{gathered}
$$

where

$$
\begin{gathered}
D_{W}=\sqrt{g_{R}^{4}+2 g_{R}^{2}\left(g_{R}^{2}-g_{L}^{2}\right) \eta+\left\{\left(g_{R}^{2}-g_{L}^{2}\right)^{2}+4 s_{2 \beta}^{2} g_{L}^{2} g_{R}^{2}\right\} \eta^{2}}, \\
c_{\omega}=\frac{1}{\sqrt{2}} \sqrt{1+\frac{g_{R}^{2}+\left(g_{R}^{2}-g_{L}^{2}\right) \eta}{D_{W}}}, \quad s_{\omega}=-\frac{1}{\sqrt{2}} \sqrt{1-\frac{g_{R}^{2}+\left(g_{R}^{2}-g_{L}^{2}\right) \eta}{D_{W}}},
\end{gathered}
$$

and $\eta:=v_{H}^{2} / v_{\Sigma}^{2}$. The rotation matrix for the neutral bosons is given by

$$
R_{Z}=\left(\begin{array}{ccc}
c_{12} & s_{12} & 0 \\
-s_{12} & c_{12} & 0 \\
0 & 0 & 1
\end{array}\right)\left(\begin{array}{ccc}
c_{13} & 0 & s_{13} \\
0 & 1 & 0 \\
-s_{13} & 0 & c_{13}
\end{array}\right)\left(\begin{array}{ccc}
1 & 0 & 0 \\
0 & c_{23} & s_{23} \\
0 & -s_{23} & c_{23}
\end{array}\right) \text {. }
$$

Here, the angles are given by

$$
c_{12}=\frac{g_{R}}{\sqrt{g_{L}^{2}+g_{R}^{2}}}, \quad s_{12}=-\frac{g_{L}}{\sqrt{g_{L}^{2}+g_{R}^{2}}}, \quad c_{13}=-\sqrt{3\left(g_{L}^{2}+g_{R}^{2}\right)} \frac{g_{4}}{\tilde{g}^{2}}, \quad s_{13}=\sqrt{2} \frac{g_{L} g_{R}}{\tilde{g}^{2}},
$$




$$
c_{23}=\frac{1}{\sqrt{2}} \sqrt{1-\frac{s-2 \tilde{g}^{4} /\left(g_{L}^{2}+g_{R}^{2}\right)}{D_{Z}}}, \quad s_{23}=\frac{1}{\sqrt{2}} \sqrt{1+\frac{s-2 \tilde{g}^{4} /\left(g_{L}^{2}+g_{R}^{2}\right)}{D_{Z}}},
$$

where

$$
\tilde{g}^{2}=\sqrt{2 g_{R}^{2} g_{L}^{2}+3 g_{4}^{2}\left(g_{L}^{2}+g_{R}^{2}\right)}, \quad s=3 g_{4}^{2}+2 g_{R}^{2}+\left(g_{L}^{2}+g_{R}^{2}\right) \eta, \quad D_{Z}=\sqrt{s^{2}-4 \tilde{g}^{4} \eta} .
$$

The $Z$ and $Z^{\prime}$ boson masses are given by

$$
m_{Z}^{2}=\frac{v_{\Sigma}^{2}}{4}\left(s-D_{Z}\right), \quad m_{Z^{\prime}}^{2}=\frac{v_{\Sigma}^{2}}{4}\left(s+D_{Z}\right) .
$$

The rotation matrices $R_{W}, R_{Z}$ diagonalize the mass matrices as

$$
R_{W}^{T} \mathcal{M}_{W}^{2} R_{W}=\operatorname{diag}\left(m_{W}^{2}, m_{W^{\prime}}^{2}\right), \quad R_{Z}^{T} \mathcal{M}_{Z}^{2} R_{Z}=\operatorname{diag}\left(0, m_{Z}^{2}, m_{Z^{\prime}}^{2}\right)
$$

If the subleading terms in $\eta$ are neglected, the vector boson masses are given by

$$
m_{W}^{2}=\frac{g_{L}^{2}}{2} v_{H}^{2}, \quad m_{W^{\prime}}^{2}=\frac{g_{R}^{2}}{2} v_{\Sigma}^{2}
$$

and

$$
m_{Z}^{2}=\frac{\tilde{g}^{4}}{3 g_{4}^{2}+2 g_{R}^{2}} \frac{v_{H}^{2}}{2}, \quad m_{Z^{\prime}}^{2}=\left(3 g_{4}^{2}+2 g_{R}^{2}\right) \frac{v_{\Sigma}^{2}}{2} .
$$

\section{b. Gauge interactions with fermions}

With the PS symmetry, the covariant derivative terms for the fermions, $\mathcal{F}_{L} \in\left(L, F_{L}, f_{L}\right)$ and $\mathcal{F}_{R} \in\left(R, f_{R}, F_{R}\right)$, are given by

$$
D^{\mu} \mathcal{F}_{L}=\partial^{\mu} \mathcal{F}_{L}-i g_{L} W_{L}^{\mu} \mathcal{F}_{L}-i g_{4} \mathcal{V}^{\mu} \mathcal{F}_{L}, \quad D^{\mu} \mathcal{F}_{R}=\partial^{\mu} R-i g_{R} W_{R}^{\mu} \mathcal{F}_{L}-i g_{4} \mathcal{V}^{\mu} \mathcal{F}_{R}
$$

The gauge couplings to the vector leptoquark are shown in Eqs. (2.19) and (2.20).

The gauge couplings with the charged $S U(2)$ bosons, $\left(W_{L}^{-}, W_{R}^{-}\right)$are given by

$$
\begin{aligned}
\mathcal{L}_{W}= & \frac{g_{L}}{\sqrt{2}} W_{L \mu}^{-}\left(\overline{\mathbf{u}}_{L} \gamma^{\mu} P_{6} \mathbf{d}_{L}+\overline{\mathbf{n}}_{L} \gamma^{\mu} P_{6} \mathbf{e}_{L}+\overline{\mathbf{u}}_{R} \gamma^{\mu} P_{\overline{6}} \mathbf{d}_{R}+\overline{\mathbf{n}}_{R} \gamma^{\mu} P_{\overline{6}} \mathbf{e}_{R}\right) \\
& +\frac{g_{R}}{\sqrt{2}} W_{R \mu}^{-}\left(\overline{\mathbf{u}}_{L} \gamma^{\mu} P_{\overline{6}} \mathbf{d}_{L}+\overline{\mathbf{n}}_{L} \gamma^{\mu} P_{\overline{6}} \mathbf{e}_{L}+\overline{\mathbf{u}}_{R} \gamma^{\mu} P_{6} \mathbf{d}_{R}+\overline{\mathbf{n}}_{R} \gamma^{\mu} P_{6} \mathbf{e}_{R}\right)+\text { H.c. } \\
= & W_{L \mu}^{-}\left(\overline{\hat{\mathbf{u}}}_{L} \hat{g}_{q_{L}}^{W_{L}} \gamma^{\mu} \hat{\mathbf{d}}_{L}+\overline{\hat{\mathbf{N}}}_{L} \hat{g}_{\ell_{L}}^{W_{L}} \gamma^{\mu} \hat{\mathbf{e}}_{L}+\overline{\hat{\mathbf{u}}}_{R} \hat{g}_{q_{R}}^{W_{L}} \gamma^{\mu} \hat{\mathbf{d}}_{R}+\overline{\mathbf{N}}_{R} \hat{g}_{\ell_{R}}^{W_{L}} \gamma^{\mu} \hat{\mathbf{e}}_{R}\right) \\
& +W_{R \mu}^{-}\left(\overline{\mathbf{u}}_{L} \hat{g}_{q_{L}}^{W_{R}} \gamma^{\mu} \hat{\mathbf{d}}_{L}+\overline{\mathbf{\mathbf { N }}}_{L} \hat{g}_{\ell_{L}}^{W_{R}} \gamma^{\mu} \hat{\mathbf{e}}_{L}+\overline{\mathbf{u}}_{R} \hat{g}_{q_{R}}^{W_{R}} \gamma^{\mu} \hat{\mathbf{d}}_{R}+\overline{\mathbf{N}}_{R} \hat{g}_{\ell_{R}}^{W_{R}} \gamma^{\mu} \hat{\mathbf{e}}_{R}\right)+\text { H.c., }
\end{aligned}
$$

where the couplings in the mass basis of the fermions are given by 


$$
\begin{aligned}
\hat{g}_{q_{L}}^{W_{L}}=\frac{g_{L}}{\sqrt{2}}\left(U_{L}^{u}\right)^{\dagger} P_{6} U_{L}^{d}, & \hat{g}_{\ell_{L}}^{W_{L}}=\frac{g_{L}}{\sqrt{2}}\left(U_{L}^{n}\right)^{\dagger} P_{6} U_{L}^{e}, \\
\hat{g}_{q_{R}}^{W_{L}}=\frac{g_{L}}{\sqrt{2}}\left(U_{R}^{u}\right)^{\dagger} P_{\overline{6}} U_{R}^{d}, & \hat{g}_{\ell_{R}}^{W_{L}}=\frac{g_{L}}{\sqrt{2}}\left(U_{R}^{n}\right)^{\dagger} P_{\overline{6}} U_{R}^{e}, \\
\hat{g}_{q_{L}}^{W_{R}}=\frac{g_{R}}{\sqrt{2}}\left(U_{L}^{u}\right)^{\dagger} P_{\overline{6}} U_{L}^{d}, & \hat{g}_{\ell_{L}}^{W_{R}}=\frac{g_{R}}{\sqrt{2}}\left(U_{L}^{n}\right)^{\dagger} P_{\overline{6}} U_{L}^{e}, \\
\hat{g}_{q_{R}}^{W_{R}}=\frac{g_{R}}{\sqrt{2}}\left(U_{R}^{u}\right)^{\dagger} P_{6} U_{R}^{d}, & \hat{g}_{\ell_{R}}^{W_{R}}=\frac{g_{R}}{\sqrt{2}}\left(U_{R}^{n}\right)^{\dagger} P_{6} U_{R}^{e} .
\end{aligned}
$$

Here, the projection matrices are defined as

$$
P_{6}:=\left(\begin{array}{cc}
\mathbf{1}_{6} & 0_{6 \times 3} \\
0_{3 \times 6} & 0_{3}
\end{array}\right), \quad P_{\overline{6}}:=\mathbf{1}_{9}-P_{6} .
$$

Note that $\left(W_{L}, W_{R}\right)$ is not a mass base of the gauge bosons. For instance, the couplings to the left-handed quarks are given by

$$
\left(\begin{array}{c}
\hat{g}_{q_{L}}^{W} \\
\hat{g}_{q_{L}}^{W^{\prime}}
\end{array}\right)=R_{W}^{T}\left(\begin{array}{c}
\hat{g}_{q_{L}}^{W_{L}} \\
\hat{g}_{q_{L}}^{W_{R}}
\end{array}\right),
$$

and those for the fermions can be obtained in the same way.

The gauge couplings with the neutral gauge bosons $\left(W_{L}^{3}, W_{R}^{3}, V^{B-L}\right)$ are given by

$$
\begin{aligned}
\mathcal{L}_{Z}= & \frac{g_{L}}{2} W_{L \mu}^{3} \sum_{\mathbf{f}=\mathbf{u}, \mathbf{d}, \mathbf{e}, \mathbf{n}} I_{\mathbf{f}}\left(\overline{\mathbf{f}}_{L} \gamma^{\mu} P_{6} \mathbf{f}_{L}+\overline{\mathbf{f}}_{R} \gamma^{\mu} P_{\overline{6}} \mathbf{f}_{R}\right) \\
& +\frac{g_{R}}{2} W_{R \mu}^{3} \sum_{\mathbf{f}=\mathbf{u}, \mathbf{d}, \mathbf{e}, \mathbf{n}} I_{\mathbf{f}}\left(\overline{\mathbf{f}}_{L} \gamma^{\mu} P_{\overline{6}} \mathbf{f}_{L}+\overline{\mathbf{f}}_{R} \gamma^{\mu} P_{6} \mathbf{f}_{R}\right) \\
& -\sqrt{\frac{3}{8}} g_{4} V_{\mu}^{B-L} \sum_{\mathbf{f}=\mathbf{u}, \mathbf{d}, \mathbf{e}, \mathbf{n}} Q_{B-L}^{\mathbf{f}}\left(\overline{\mathbf{f}}_{L} \gamma^{\mu} \mathbf{f}_{L}+\overline{\mathbf{f}}_{R} \gamma^{\mu} \mathbf{f}_{R}\right) \\
= & \sum_{V=W_{L}^{3}, W_{R}^{3}, V^{B-L}} V_{\mu} \sum_{\mathbf{f}=\mathbf{u}, \mathbf{d}, \mathbf{e}, \mathbf{N}}\left(\overline{\mathbf{f}}_{L} \hat{g}_{\mathbf{f}_{L}}^{V} \gamma^{\mu} \hat{\mathbf{f}}_{L}+\overline{\mathbf{f}}_{R} \hat{g}_{\mathbf{f}_{R}}^{V} \gamma^{\mu} \hat{\mathbf{f}}_{R}\right),
\end{aligned}
$$

where $I_{\mathbf{f}}=+1(-1)$ for $\mathbf{f}=\mathbf{e}, \mathbf{d}(\mathbf{u}, \mathbf{n}) . Q_{B-L}^{\mathbf{f}}$ is the $B-L$ number of a fermion $\mathbf{f}$. The gauge coupling matrices in the fermion mass basis are given by

$$
\begin{aligned}
\hat{g}_{f_{L}}^{W_{L}^{3}}=\frac{g_{L}}{2} I_{L}^{f}\left(U_{L}^{f}\right)^{\dagger} P_{6} U_{L}^{f}, & \hat{g}_{f_{R}}^{W_{L}^{3}}=\frac{g_{L}}{2} I_{L}^{f}\left(U_{R}^{f}\right)^{\dagger} P_{\overline{6}} U_{R}^{f}, \\
\hat{g}_{f_{L}}^{W_{R}^{3}}=\frac{g_{R}}{2} I_{R}^{f}\left(U_{L}^{f}\right)^{\dagger} P_{\overline{6}} U_{L}^{f}, & \hat{g}_{f_{R}}^{W_{R}^{3}}=\frac{g_{R}}{2} I_{R}^{f}\left(U_{R}^{f}\right)^{\dagger} P_{6} U_{R}^{f}, \\
\hat{g}_{f_{L}}^{V^{B-L}}=-\sqrt{\frac{3}{8}} g_{4} Q_{B-L}^{f}\left(U_{L}^{f}\right)^{\dagger} U_{L}^{f}, & \hat{g}_{f_{R}}^{V^{B-L}}=-\sqrt{\frac{3}{8}} g_{4} Q_{B-L}^{f}\left(U_{R}^{f}\right)^{\dagger} U_{R}^{f} .
\end{aligned}
$$

The coupling matrices in the mass basis are

$$
\left(\begin{array}{c}
\hat{g}_{f_{L}}^{A} \\
\hat{g}_{f_{L}}^{Z} \\
\hat{g}_{f_{L}^{\prime}}^{Z^{\prime}}
\end{array}\right)=R_{Z}^{T}\left(\begin{array}{c}
\hat{g}_{f_{L}}^{W_{L}^{3}} \\
\hat{g}_{f_{L}}^{W_{R}^{3}} \\
\hat{g}_{f_{L} V^{B-L}}^{3}
\end{array}\right), \quad\left(\begin{array}{c}
\hat{g}_{f_{R}}^{A} \\
\hat{g}_{f_{R}}^{Z} \\
\hat{g}_{f_{R}}^{Z^{\prime}}
\end{array}\right)=R_{Z}^{T}\left(\begin{array}{c}
\hat{g}_{f_{R}}^{W_{L}^{3}} \\
\hat{g}_{f_{R}}^{W_{R}^{3}} \\
\hat{g}_{f_{R}}^{V^{B-L}}
\end{array}\right) \text {. }
$$


Approximately, $R_{W}$ and $R_{Z}$ are given by

$$
R_{W}=\left(\begin{array}{ll}
1 & 0 \\
0 & 1
\end{array}\right)+\mathcal{O}(\eta), \quad R_{Z}=\left(\begin{array}{ccc}
-\sqrt{3} g_{4} g_{R} / \tilde{g}^{2} & -\sqrt{s} g_{L} / \tilde{g}^{2} & 0 \\
-\sqrt{3} g_{4} g_{L} / \tilde{g}^{2} & 3 g_{4}^{2} g_{R} /\left(\sqrt{s} \tilde{g}^{2}\right) & \sqrt{2} g_{R} / \sqrt{s} \\
-\sqrt{2} g_{L} g_{R} / \tilde{g}^{2} & \sqrt{6} g_{4} g_{R}^{2} /\left(\sqrt{s} \tilde{g}^{2}\right) & -\sqrt{3} g_{4} / \sqrt{s}
\end{array}\right)+\mathcal{O}(\eta)
$$

At the leading order in $\eta, W \sim W_{L}$ and $W^{\prime} \sim W_{R}$. The coupling matrices to the fermions are approximately given by

$$
\begin{aligned}
& \hat{g}_{q_{L}}^{W} \sim \frac{g_{L}}{\sqrt{2}}\left(\begin{array}{ccc}
u_{u_{L}}^{\dagger} & 0_{3} & 0_{3} \\
0_{3} & \mathbf{1}_{3} & 0_{3} \\
0_{3} & 0_{3} & 0_{3}
\end{array}\right), \quad \hat{g}_{q_{R}}^{W} \sim \frac{g_{L}}{\sqrt{2}}\left(\begin{array}{ccc}
0_{3} & 0_{3} & 0_{3} \\
0_{3} & \mathbf{1}_{3} & 0_{3} \\
0_{3} & 0_{3} & 0_{3}
\end{array}\right), \\
& \mathcal{P}_{L} \hat{g}_{\ell_{L}}^{W} \sim \frac{g_{L}}{\sqrt{2}}\left(\begin{array}{ccc}
u_{n_{L}}^{\dagger} & 0_{3} & 0_{3} \\
0_{3} & 0_{3} & 0_{3} \\
0_{3} & 0_{3} & \mathbf{1}_{3}
\end{array}\right), \quad \mathcal{P}_{R} \hat{g}_{\ell_{R}}^{W} \sim \frac{g_{L}}{\sqrt{2}}\left(\begin{array}{ccc}
0_{3} & 0_{3} & 0_{3} \\
0_{3} & 0_{3} & 0_{3} \\
0_{3} & 0_{3} & \mathbf{1}_{3}
\end{array}\right), \\
& \hat{g}_{q_{L}}^{W^{\prime}} \sim \frac{g_{R}}{\sqrt{2}}\left(\begin{array}{ccc}
0_{3} & 0_{3} & 0_{3} \\
0_{3} & 0_{3} & 0_{3} \\
0_{3} & 0_{3} & \mathbf{1}_{3}
\end{array}\right), \quad \hat{g}_{q_{R}}^{W^{\prime}} \sim \frac{g_{R}}{\sqrt{2}}\left(\begin{array}{ccc}
u_{u_{R}}^{\dagger} & 0_{3} & 0_{3} \\
0_{3} & 0_{3} & 0_{3} \\
0_{3} & 0_{3} & \mathbf{1}_{3}
\end{array}\right), \\
& \mathcal{P}_{L} \hat{g}_{\ell_{L}}^{W^{\prime}} \sim \frac{g_{R}}{\sqrt{2}}\left(\begin{array}{ccc}
0_{3} & 0_{3} & 0_{3} \\
0_{3} & \mathbf{1}_{3} & 0_{3} \\
0_{3} & 0_{3} & 0_{3}
\end{array}\right), \quad \mathcal{P}_{R} \hat{g}_{\ell_{R}}^{W^{\prime}} \sim \frac{g_{R}}{\sqrt{2}}\left(\begin{array}{ccc}
u_{n_{R}}^{\dagger} & 0_{3} & 0_{3} \\
0_{3} & \mathbf{1}_{3} & 0_{3} \\
0_{3} & 0_{3} & 0_{3}
\end{array}\right) \text {. }
\end{aligned}
$$

The other blocks in the neutrino couplings are vanishing. To the SM families, the $W$ boson couples via the left current, while the $W^{\prime}$ boson couples via the right current. Their flavor structure depends on independent unitary matrices, $u_{u_{L, R}}$ and $u_{n_{L, R}}$. In particular, $u_{u_{L}}^{\dagger}$ and $u_{n_{L}}^{\dagger}$ correspond to the CKM and PMNS matrices, respectively.

The coupling matrices to the neutral bosons $\left(W_{L}^{3}, W_{R}^{3}, V^{B-L}\right)$ are given by

$$
\begin{array}{rlrl}
\hat{g}_{q_{L}}^{W_{L}^{3}} \sim \frac{g_{L}}{2} I_{q} P_{q_{L}}^{Z}, & \hat{g}_{q_{L}}^{W_{R}^{3}} \sim \frac{g_{R}}{2} I_{q} P_{q_{L}}^{Z^{\prime}}, & \hat{g}_{q_{L}}^{V^{B-L}} \sim-g_{4} \sqrt{\frac{1}{24}} \mathbf{1}_{9}, \\
\hat{g}_{q_{R}}^{W_{L}^{3}} \sim \frac{g_{L}}{2} I_{q} P_{q_{R}}^{Z}, & \hat{g}_{q_{R}}^{W_{R}^{3}} \sim \frac{g_{R}}{2} I_{q} P_{q_{R}}^{Z^{\prime}}, & \hat{g}_{q_{R}}^{V^{B-L}} \sim-g_{4} \sqrt{\frac{1}{24}} \mathbf{1}_{9}, \\
\hat{g}_{\ell_{L}}^{W_{L}^{3}} \sim \frac{g_{L}}{2} I_{\ell} P_{\ell_{L}}^{Z}, & \hat{g}_{\ell_{L}}^{W_{R}^{3}} \sim \frac{g_{R}}{2} I_{\ell} P_{\ell_{L}}^{Z^{\prime}}, & \hat{g}_{\ell_{L}}^{V^{B-L}} \sim g_{4} \sqrt{\frac{3}{8}} \mathbf{1}_{9}, \\
\hat{g}_{\ell_{R}}^{W_{L}^{3}} \sim \frac{g_{L}}{2} I_{\ell} P_{\ell_{R}}^{Z}, & \hat{g}_{\ell_{R}}^{W_{R}^{3}} \sim \frac{g_{R}}{2} I_{\ell} P_{\ell_{R}}^{Z^{\prime}}, & \hat{g}_{\ell_{R}}^{V^{B-L}} & \sim g_{4} \sqrt{\frac{3}{8}} \mathbf{1}_{9},
\end{array}
$$

where $q=u, d$ and $\ell=e, n$. The coupling matrices are given by

$$
P_{q_{L}}^{Z}=P_{\ell_{R}}^{Z^{\prime}}=\left(\begin{array}{ccc}
\mathbf{1}_{3} & 0_{3} & 0_{3} \\
0_{3} & \mathbf{1}_{3} & 0_{3} \\
0_{3} & 0_{3} & 0_{3}
\end{array}\right), \quad P_{q_{R}}^{Z}=P_{\ell_{L}}^{Z^{\prime}}=\left(\begin{array}{ccc}
0_{3} & 0_{3} & 0_{3} \\
0_{3} & \mathbf{1}_{3} & 0_{3} \\
0_{3} & 0_{3} & 0_{3}
\end{array}\right)
$$




$$
P_{\ell_{L}}^{Z}=P_{q_{R}}^{Z^{\prime}}=\left(\begin{array}{ccc}
\mathbf{1}_{3} & 0_{3} & 0_{3} \\
0_{3} & 0_{3} & 0_{3} \\
0_{3} & 0_{3} & \mathbf{1}_{3}
\end{array}\right), \quad P_{\ell_{R}}^{Z}=P_{q_{L}}^{Z^{\prime}}=\left(\begin{array}{ccc}
0_{3} & 0_{3} & 0_{3} \\
0_{3} & 0_{3} & 0_{3} \\
0_{3} & 0_{3} & \mathbf{1}_{3}
\end{array}\right)
$$

For the neutrinos, these are the $9 \times 9$ upper-left (bottom-right) block in the $18 \times 18$ coupling matrices for the left-handed (right-handed) neutrinos. Using Eq. (A49), the gauge couplings to the neutral gauge bosons in the mass basis, $\left(A, Z, Z^{\prime}\right)$ are approximately given by

$$
\hat{g}_{f_{X}}^{A} \sim e Q_{e}^{f} \mathbf{1}_{9}, \quad \hat{g}_{f_{X}}^{Z} \sim \frac{g_{L}}{c_{W}}\left(-\frac{I_{f}}{2} P_{f_{X}}^{Z}-s_{W}^{2} Q_{e}^{f} \mathbf{1}_{9}\right), \quad \hat{g}_{f_{X}}^{Z^{\prime}} \sim \frac{g_{Z^{\prime}}}{2}\left(s_{Z^{\prime}}^{2} P_{f_{X}}^{Z^{\prime}} I_{f}+c_{Z^{\prime}}^{2} Q_{B-L}^{f} \mathbf{1}_{9}\right),
$$

where the electric charge, coupling constant and $Z^{\prime}$ gauge coupling constant are given by

$$
Q_{e}^{f}=\frac{Q_{B-L}^{f}-I_{f}}{2}, \quad e=\sqrt{3} \frac{g_{4} g_{L} g_{R}}{\tilde{g}^{2}}, \quad g_{Z^{\prime}}=\sqrt{\frac{2 g_{R}^{2}+3 g_{4}^{2}}{2}} .
$$

For the $Z$ and $Z^{\prime}$ boson couplings, the angles are defined as

$$
c_{W}=\frac{g_{L} \sqrt{3 g_{4}^{2}+2 g_{R}^{2}}}{\tilde{g}^{2}}, \quad s_{W}=\frac{\sqrt{3} g_{4} g_{R}}{\tilde{g}^{2}}, \quad c_{Z^{\prime}}=\sqrt{\frac{2 g_{R}^{2}}{2 g_{R}^{2}+3 g_{4}^{2}}}, \quad s_{Z^{\prime}}=\sqrt{\frac{3 g_{4}^{2}}{2 g_{R}^{2}+3 g_{4}^{2}}} .
$$

Therefore, the EW gauge couplings coincide with the SM values when $\mathcal{O}(\eta)$ effects are negligible.

In the above approximate formulas, we neglected $\mathcal{O}\left(v_{H}^{2} / v_{\Delta}^{2}, \eta\right)$ effects in the diagonalization unitary matrices for the fermion and gauge boson mass matrices. In the fermion mass matrix, the $\mathcal{O}\left(v_{H}\right)$ elements, denoted by $\cdot$ in Eq. (A3), in the off-diagonal blocks are neglected. These off-diagonal entries will induce flavor violating couplings with the EW gauge bosons. The flavor violating coupling to $f_{i} f_{j}$ will be $\mathcal{O}\left(g_{L}\left[d_{f}\right]_{i}\left[d_{f}\right]_{j} / v_{\Delta}^{2}\right)$, where $\left[d_{f}\right]_{i}$ is the mass of the SM fermion of the $i$ th generation. ${ }^{15}$ Here we assume that all the vectorlike fermion masses are $\mathcal{O}\left(v_{\Delta}\right)$. If $v_{\Delta} \sim$ $\mathcal{O}(10 \mathrm{TeV})$ as considered in this paper, the induced flavor violating coupling is at most $\mathcal{O}\left(10^{-6}\right)$ for top and charm quarks, and the smaller for the light flavor fermions. Thus the flavor violation from the EW gauge bosons is too small to be measured by experiments. The mixing of $Z-Z^{\prime}$ and $W-W^{\prime}$ will affect EW precision observables, since these induce exotic right-current interactions. Again, when $v_{\Sigma} \sim \mathcal{O}(10 \mathrm{TeV})$, the effect is $\mathcal{O}\left(10^{-4}\right)$, and thus may be too small to be measured. Note that $Z^{\prime}$ and $W^{\prime}$ can be lighter while keeping the leptoquark $\mathcal{O}(10 \mathrm{TeV})$ if $v_{\Sigma} \ll v_{\Delta}$. This would be an interesting possibility but is beyond the scope of this paper. In our numerical analysis, flavor violating effects from the extra gauge bosons are neglected.

\section{Scalar interactions}

In our model, there are three scalar fields $\Delta, \Sigma$ and $\Phi$ introduced to break the PS to SM symmetry. In this paper,

\footnotetext{
${ }^{15}$ See Refs. $[61,62]$ for the similar analysis in a model with one vectorlike generation.
}

we will not consider the scalar potential explicitly, and we assume that the scalar potential has the global minimum at the VEVs which we assumed.

The EW symmetry is broken by the VEV of bi-doublet $\Phi$,

$$
\Phi=\left(\begin{array}{cc}
H_{1}^{0} & H_{2}^{+*} \\
H_{1}^{+} & -H_{2}^{0 *}
\end{array}\right)=\left(\begin{array}{ll}
H_{1} & \tilde{H}_{2}
\end{array}\right)
$$

where $H_{1,2}$ are the $S U(2)_{L}$ doublets which can be expanded as

$$
H_{k}=\left(\begin{array}{c}
H_{k}^{0} \\
H_{k}^{+}
\end{array}\right)=\frac{1}{\sqrt{2}}\left(\begin{array}{c}
v_{k}+h_{k}+i a_{k} \\
\sqrt{2} H_{k}^{+}
\end{array}\right), \quad k=1,2 .
$$

The mass basis of the doublet Higgs bosons is defined as

$$
\begin{aligned}
\left(\begin{array}{l}
h_{1} \\
h_{2}
\end{array}\right) & =R_{\alpha}\left(\begin{array}{l}
h \\
H
\end{array}\right), \quad\left(\begin{array}{l}
a_{1} \\
a_{2}
\end{array}\right)=R_{\beta_{0}}\left(\begin{array}{c}
G^{0} \\
A
\end{array}\right), \\
\left(\begin{array}{l}
H_{1}^{+} \\
H_{2}^{+}
\end{array}\right) & =R_{\beta_{+}}\left(\begin{array}{l}
G^{+} \\
H^{+}
\end{array}\right),
\end{aligned}
$$

where $G^{0}, G^{+}$are the Nambu-Goldstone bosons. The rotation matrices are defined with the angles as

$$
R_{\phi}=\left(\begin{array}{cc}
\cos \phi & -\sin \phi \\
\sin \phi & \cos \phi
\end{array}\right), \quad \phi=\alpha, \beta_{0}, \beta_{ \pm}
$$

In the decoupling limit $\left(m_{A}, m_{H^{ \pm}} \gg m_{h}\right)$, these angles are aligned as $\alpha=\beta_{0}=\beta_{ \pm}=\beta$ where $\tan \beta \equiv v_{2} / v_{1}$. The Higgs couplings to the fermions are given by 


$$
\begin{aligned}
-\mathcal{L}_{H}= & \sum_{S=h, H, A} S\left(\overline{\mathbf{u}}_{L} Y_{\mathbf{u}}^{S} \mathbf{u}_{R}+\overline{\mathbf{d}}_{L} Y_{d}^{S} \mathbf{d}_{R}+\overline{\mathbf{e}}_{L} Y_{e}^{S} \mathbf{e}_{R}+\overline{\mathbf{n}}_{L} Y_{n}^{S} \mathbf{n}_{R}\right) \\
& +H^{+}\left(\overline{\mathbf{u}}_{L} Y_{d}^{H^{+}} \mathbf{d}_{R}+\overline{\mathbf{n}}_{L} Y_{e}^{H^{+}} \mathbf{e}_{R}\right) \\
& +H^{-}\left(\overline{\mathbf{d}}_{L} Y_{u}^{H^{-}} \mathbf{u}_{R}+\overline{\mathbf{e}}_{L} Y_{n}^{H^{-}} \mathbf{n}_{R}\right)+\text { H.c. }
\end{aligned}
$$

The Yukawa matrices $Y_{f}^{S}$ are given by linear combinations of

$$
Y_{a}=\left(\begin{array}{ccc}
y_{a} & \tilde{\lambda}_{a} & 0_{3} \\
\lambda_{a} & \tilde{y}_{a} & 0_{3} \\
0_{3} & 0_{3} & \tilde{y}_{a}^{\prime}
\end{array}\right), \quad a=1,2
$$

where the $3 \times 3$ Yukawa matrices are defined in Eqs. (2.1) and (2.2). The relation of these Yukawa matrices to the quark Yukawa matrix is given by

$$
\left(\begin{array}{c}
Y_{d} \\
Y_{u}
\end{array}\right):=\frac{d}{d v_{H}}\left(\begin{array}{c}
\mathcal{M}_{d} \\
\mathcal{M}_{u}
\end{array}\right)=\left(\begin{array}{cc}
c_{\beta} & -s_{\beta} \\
-s_{\beta} & c_{\beta}
\end{array}\right)\left(\begin{array}{c}
Y_{1} \\
Y_{2}
\end{array}\right)
$$

where $Y_{d}$ and $Y_{u}$ are the Yukawa matrices aligned to the relevant block of the mass matrix. The Yukawa matrices for $S=h, H, A, H^{ \pm}$are given by

$$
\left(\begin{array}{c}
Y_{d}^{h} \\
Y_{u}^{h}
\end{array}\right)=\frac{1}{\sqrt{2} \cos 2 \beta}\left(\begin{array}{cc}
\cos (\alpha+\beta) & -\sin (\alpha-\beta) \\
-\sin (\alpha-\beta) & \cos (\alpha+\beta)
\end{array}\right)\left(\begin{array}{c}
Y_{d} \\
Y_{u}
\end{array}\right),
$$

$$
\left(\begin{array}{c}
Y_{d}^{H} \\
Y_{u}^{H}
\end{array}\right)=\frac{-1}{\sqrt{2} \cos 2 \beta}\left(\begin{array}{cc}
\sin (\alpha+\beta) & \cos (\alpha-\beta) \\
\cos (\alpha-\beta) & \sin (\alpha+\beta)
\end{array}\right)\left(\begin{array}{c}
Y_{d} \\
Y_{u}
\end{array}\right)
$$

$$
\left(\begin{array}{c}
Y_{d}^{A} \\
Y_{u}^{A}
\end{array}\right)=\frac{-i}{\sqrt{2} \cos 2 \beta}\left(\begin{array}{cc}
\sin \left(\beta_{0}+\beta\right) & \cos \left(\beta_{0}-\beta\right) \\
-\cos \left(\beta_{0}-\beta\right) & -\sin \left(\beta_{0}+\beta\right)
\end{array}\right)\left(\begin{array}{c}
Y_{d} \\
Y_{u}
\end{array}\right),
$$

$$
\left(\begin{array}{c}
Y_{d}^{H^{+}} \\
Y_{u}^{H^{-}}
\end{array}\right)=\frac{-1}{\cos 2 \beta}\left(\begin{array}{cc}
\sin \left(\beta_{ \pm}+\beta\right) & \cos \left(\beta_{ \pm}-\beta\right) \\
-\cos \left(\beta_{ \pm}-\beta\right) & -\sin \left(\beta_{ \pm}+\beta\right)
\end{array}\right)\left(\begin{array}{c}
Y_{d} \\
Y_{u}
\end{array}\right)
$$

In the gauge basis, the lepton Yukawa couplings are the same as those of the quarks, e.g., $Y_{d}^{h}=Y_{e}^{h}$. The Yukawa matrices in the mass basis are given by

$$
\hat{Y}_{f}^{S^{0}}=\left(U_{L}^{f}\right)^{\dagger} Y_{f}^{S^{0}} U_{R}^{f},
$$

where $S^{0}=h, H, A$ and $f=u, d, e, n$. The charge Higgs couplings are given by
$\hat{Y}_{d}^{H^{+}}=\left(U_{L}^{u}\right)^{\dagger} Y_{d}^{H^{+}} U_{R}^{d}, \quad \hat{Y}_{u}^{H^{-}}=\left(U_{L}^{d}\right)^{\dagger} Y_{u}^{H^{-}} U_{R}^{u}$,

$\hat{Y}_{e}^{H^{+}}=\left(U_{L}^{n}\right)^{\dagger} Y_{e}^{H^{+}} U_{R}^{e}, \quad \hat{Y}_{n}^{H^{-}}=\left(U_{L}^{e}\right)^{\dagger} Y_{n}^{H^{-}} U_{R}^{n}$.

In the decoupling limit, the SM Higgs couplings are aligned with the mass matrix, i.e., $Y_{d(u)}^{h} \propto Y_{d(u)}$, while the Yukawa couplings to the heavier Higgs bosons are not. Therefore the heavy Higgs bosons generically induce flavor violation.

The $S U(4)_{C}$ adjoint scalar $\Delta$ is expanded as

$\Delta=\frac{1}{2 \sqrt{3}}\left(v_{\Delta}+\frac{h_{\Delta}}{\sqrt{2}}\right)\left(\begin{array}{cc}3 & 0 \\ 0 & -\mathbf{1}_{3}\end{array}\right)+\left(\begin{array}{cc}0 & 0 \\ 0 & \Delta_{8}\end{array}\right)$.

Here, $h_{\Delta}$ is a $C P$-even neutral scalar and $\Delta_{8}$ is a $S U(3)_{C}$ adjoint scalar field. The Yukawa couplings involving $\Delta$ and $\Delta_{8}$ are given by

$$
\begin{aligned}
-\mathcal{L}_{\Delta}= & \sum_{\mathbf{f}=\mathbf{u}, \mathbf{d}, \mathbf{e}, \mathbf{n}} \sqrt{\frac{3}{8}} h_{\Delta} Q_{B-L}^{\mathbf{f}} \overline{\mathbf{f}}_{L} Y_{\Delta} \mathbf{f}_{R} \\
& +\sum_{\mathbf{f}=\mathbf{u}, \mathbf{d}} \overline{\mathbf{f}}_{L} Y_{\Delta} \Delta_{8} \mathbf{f}_{R}+\text { H.c. }
\end{aligned}
$$

where the Yukawa coupling matrices in the gauge basis are common for the fermions,

$$
Y_{\Delta}=\left(\begin{array}{ccc}
0_{3} & 0_{3} & \epsilon_{L} \\
0_{3} & 0_{3} & \kappa_{L} \\
\epsilon_{R} & \kappa_{R} & 0_{3}
\end{array}\right)=\frac{\sqrt{3}}{2 v_{\Delta}}\left(\mathcal{M}_{e}-\mathcal{M}_{d}\right)
$$

In the mass basis, the couplings are given by

$$
\hat{Y}_{\Delta}^{f}=\left(U_{L}^{f}\right)^{\dagger} Y_{\Delta} U_{R}^{f}, \quad f=u, d, e, n .
$$

The flavor violation is also induced by the $\Delta$ couplings, although the sizable contributions appear only with vectorlike generations. For instance, the Yukawa coupling to the charged leptons is approximately given by

$$
\hat{Y}_{\Delta}^{e} \sim\left(\tilde{U}_{e_{L}}\right)^{\dagger} Y_{\Delta} \tilde{U}_{e_{R}}=\left(\begin{array}{ccc}
0_{3} & 0_{3} & \Lambda_{L_{1}}^{e} \\
\Lambda_{R_{2}}^{e} & \Lambda_{R_{1}}^{e} & 0_{3} \\
0_{3} & 0_{3} & \Lambda_{L_{2}}^{e}
\end{array}\right)+\mathcal{O}\left(\frac{v_{H}}{v_{\Delta}}\right),
$$

where the $3 \times 3$ coupling matrices $\Lambda_{L, R_{1,2}}^{e}$ are given by

$$
\begin{gathered}
\left(\begin{array}{cc}
\Lambda_{R_{1}}^{e} & \Lambda_{R_{2}}^{e}
\end{array}\right)=\tilde{D}_{\ell_{R}}-W_{\ell_{L}}^{\dagger} W_{Q_{L}} \tilde{D}_{Q_{R}} V_{Q_{R}}^{\dagger} V_{\ell_{R}}, \\
\left(\begin{array}{c}
\Lambda_{L_{1}}^{e} \\
\Lambda_{L_{2}}^{e}
\end{array}\right)=\tilde{D}_{\ell_{L}}-V_{\ell_{L}}^{\dagger} V_{Q_{L}} \tilde{D}_{Q_{L}} W_{Q_{R}}^{\dagger} W_{\ell_{R}} .
\end{gathered}
$$

Thus, there is no coupling of $\Delta$ with two SM fermions at the leading order. It might be possible that loop effects mediated by the vectorlike fermions induce flavor 
violations, such as $\mu \rightarrow e \gamma$. For $\mu \rightarrow e \gamma$, the chirality enhanced effect enhanced by $v_{H}$ will be proportional to $\left[\hat{Y}_{\Delta}^{e}\right]_{1 a}\left[\hat{Y}_{\Delta}^{e}\right]_{a 2}, \quad a=1,2, \ldots, 9$, which are all zero in Eq. (A80). Hence, we expect that $\Delta$ will not give significant flavor violating effects no matter how the leptoquark couplings are.
APPENDIX B: ANALYSIS DETAILS

\section{Formulas of flavor observables}

a. $K_{L} \rightarrow \mu e$

The branching fraction of $K_{L} \rightarrow e_{i} e_{j}$ is given by

$$
\begin{aligned}
\operatorname{BR}\left(K_{L} \rightarrow e_{i} e_{j}\right)= & \frac{\tau_{K_{L}}}{512 \pi m_{X}^{4}}\left(m_{e_{i}}+m_{e_{j}}\right)^{2} m_{K} f_{K}^{2} \sqrt{\left(1-\frac{\left(m_{e_{i}}+m_{e_{j}}\right)^{2}}{m_{K}^{2}}\right)\left(1-\frac{\left(m_{e_{i}}-m_{e_{j}}\right)^{2}}{m_{K}^{2}}\right)} \\
& \times\left[\mid\left[\hat{g}_{d_{L}}^{X}\right]_{2 i}\left[\hat{g}_{d_{L}}^{X}\right]_{1 j}^{*}+\left[\hat{g}_{d_{R}}^{X}\right]_{2 i}\left[\hat{g}_{d_{R}}^{X}\right]_{1 j}^{*}\right. \\
& -\left.\frac{2 m_{K}^{2}}{\left(m_{e_{i}}+m_{e_{j}}\right)\left(m_{d}+m_{s}\right)}\left(\left[\hat{g}_{d_{R}}^{X}\right]_{2 i}\left[\hat{g}_{d_{L}}^{X}\right]_{1 j}^{*}+\left[\hat{g}_{d_{L}}^{X}\right]_{2 i}\left[\hat{g}_{d_{R}}^{X}\right]_{1 j}^{*}\right)\right|^{2}\left(1-\frac{\left(m_{e_{i}}-m_{e_{j}}\right)^{2}}{m_{K}^{2}}\right) \\
& \left.+\left|\frac{2 m_{K}^{2}}{\left(m_{e_{i}}+m_{e_{j}}\right)\left(m_{d}+m_{s}\right)}\left(\left[\hat{g}_{d_{R}}^{X}\right]_{2 i}\left[\hat{g}_{d_{L}}^{X}\right]_{1 j}^{*}-\left[\hat{g}_{d_{L}}^{X}\right]_{2 i}\left[\hat{g}_{d_{R}}^{X}\right]_{1 j}^{*}\right)\right|^{2}\left(1-\frac{\left(m_{e_{i}}+m_{e_{j}}\right)^{2}}{m_{K}^{2}}\right)\right] \\
& +(i \leftrightarrow j),
\end{aligned}
$$

where $m_{e_{i(j)}}$ is the masses of the $i(j)$ th generation charged lepton. In our numerical analysis, we included contributions from the Higgs bosons and adjoint scalar $h_{\Delta}, \Delta_{8}$, but we have seen that these are always negligible compared with those from the leptoquark due to the small flavor violating coupling to the charged leptons as discussed in Appendix A. We use the same formula for the other leptonic decays of $B, B_{s}$ and $B_{c}$ mesons by formally replacing coupling matrices and flavor indices appropriately.
The values of constants used in our numerical analysis are shown in Table II, and the values of observables at the benchmark point are shown in the next section.

\section{b. $\mu$-e conversion}

For the flavor violation involving the electron, $\mu-e$ conversion is also severely constrained particularly in the future experiments. The conversion rate is given by [113]

$$
\Gamma_{\text {conv }}=4 m_{\mu}^{5}\left(\left|\sum_{N=p, n}\left(\tilde{C}_{V L}^{N} V_{N}+m_{N} \tilde{C}_{S L}^{N} S_{N}\right)\right|^{2}+\left|\sum_{N=p, n}\left(\tilde{C}_{V R}^{N} V_{N}+m_{N} \tilde{C}_{S R}^{N} S_{N}\right)\right|^{2}\right)
$$

where

$$
\begin{array}{ll}
\tilde{C}_{V L}^{N}=\sum_{q=u, d, s} C_{V L}^{q} f_{V_{N}}^{q}, & \tilde{C}_{S L}^{N}=\sum_{q=u, d, s} C_{S L}^{q} f_{S_{N}}^{q}+\frac{2}{27} f_{G}^{N} \sum_{Q=c, b, t} C_{S L}^{Q}, \\
\tilde{C}_{V R}^{N}=\sum_{q=u, d, s} C_{V R}^{q} f_{V_{N}}^{q}, & \tilde{C}_{S R}^{N}=\sum_{q=u, d, s} C_{S R}^{q} f_{S_{N}}^{q}+\frac{2}{27} f_{G}^{N} \sum_{Q=c, b, t} C_{S R}^{Q} .
\end{array}
$$

The values for form factors are shown in Table III and $f_{G}^{N}=1-\sum_{q=u, d, s} f_{S_{N}}^{q}$. In our model, the coefficients are given by

TABLE II. Values of parameters of the mesons taken from PDG and HFLAG2019 [112]. The units of masses and decay constants are $\mathrm{GeV}$, that of lifetime is $\mathrm{GeV}^{-1}$.

\begin{tabular}{llllllll}
\hline \hline$m_{B_{d}}$ & 5.280 & $\tau_{B_{d}} \times 10^{-12}$ & 2.3230 & $f_{B_{d}}$ & 0.1920 & $B_{B_{d}}$ & 1.30 \\
$m_{B_{s}}$ & 5.367 & $\tau_{B_{s}} \times 10^{-12}$ & 2.2930 & $f_{B_{s}}$ & 0.2284 & $B_{B_{s}}$ & 1.35 \\
$m_{B_{c}}$ & 6.275 & $\tau_{B_{c}} \times 10^{-12}$ & 0.7703 & $f_{B_{c}}$ & 0.4340 & $\ldots$ & $\ldots$ \\
$m_{K}$ & 0.4976 & $\tau_{K_{L}} \times 10^{-17}$ & 7.7730 & $f_{K}$ & 0.1552 & $B_{K}$ & 0.717 \\
\hline \hline
\end{tabular}


TABLE III. Values of vector [113] and scalar [114] form factors. The coefficients $S_{N}, V_{N}$ are calculated in Ref. [115]. The capture rates are given in Refs. [115,116].

\begin{tabular}{lcccc}
\hline \hline$f_{V_{p}}^{u}$ & $f_{V_{p}}^{d}$ & $f_{V_{n}}^{u}$ & $f_{V_{n}}^{d}$ & $f_{V_{p}}^{s}=f_{V_{n}}^{s}$ \\
2 & 1 & 1 & 2 & 0 \\
$f_{S_{p}}^{u}$ & $f_{S_{p}}^{d}$ & $f_{S_{n}}^{u}$ & $f_{S_{n}}^{d}$ & $f_{S_{p}}^{s}=f_{S_{n}}^{s}$ \\
0.0191 & 0.0363 & 0.0171 & 0.0404 & 0.043
\end{tabular}

\begin{tabular}{lccccc}
\hline \hline Target & $S_{p}$ & $S_{n}$ & $V_{p}$ & $V_{n}$ & $\Gamma_{\text {capt }}\left[10^{6} \cdot s^{-1}\right]$ \\
\hline $\mathrm{Au}$ & 0.0614 & 0.0918 & 0.0974 & 0.146 & 13.07 \\
$\mathrm{Al}$ & 0.0155 & 0.0167 & 0.0161 & 0.0173 & 0.705 \\
\hline \hline
\end{tabular}

$$
C_{V L}^{d_{i}}=\frac{\left[\hat{g}_{d_{L}}^{X}\right]_{i 1}^{*}\left[\hat{g}_{d_{L}}^{X}\right]_{i 2}}{2 m_{X}^{2}}, \quad C_{V R}^{d_{i}}=\frac{\left[\hat{g}_{d_{R}}^{X}\right]_{i 1}^{*}\left[\hat{g}_{d_{R}}^{X}\right]_{i 2}}{2 m_{X}^{2}}
$$

and

$$
\begin{gathered}
C_{S L}^{d_{i}}=-\frac{1}{m_{d_{i}}}\left[\frac{\left[\hat{g}_{d_{L}}^{X}\right]_{i 1}^{*}\left[\hat{g}_{d_{R}}^{X}\right]_{i 2}}{m_{X}^{2}}+\sum_{S} \frac{1}{2 m_{S}^{2}}\left[\hat{Y}_{e}^{S}\right]_{12} \operatorname{Re}\left(\left[\hat{Y}_{d}^{S}\right]_{i i}\right)\right], \\
C_{S R}^{d_{i}}=-\frac{1}{m_{d_{i}}}\left[\frac{\left.\hat{g}_{d_{R}}^{X}\right]_{i 1}^{*}\left[\hat{g}_{d_{L}}^{X}\right]_{i 2}}{m_{X}^{2}}+\sum_{S} \frac{1}{2 m_{S}^{2}}\left[\hat{Y}_{e}^{S}\right]_{21}^{*} \operatorname{Re}\left(\left[\hat{Y}_{d}^{S}\right]_{i i}\right)\right], \\
C_{S L}^{u_{i}}=-\frac{1}{m_{u_{i}}} \sum_{S} \frac{1}{2 m_{S}^{2}}\left[\hat{Y}_{e}^{S}\right]_{12} \operatorname{Re}\left(\left[\hat{Y}_{u}^{S}\right]_{i i}\right), \\
C_{S R}^{u_{i}}=-\frac{1}{m_{u_{i}}} \sum_{S} \frac{1}{2 m_{S}^{2}}\left[\hat{Y}_{e}^{S}\right]_{21}^{*} \operatorname{Re}\left(\left[\hat{Y}_{u}^{S}\right]_{i i}\right),
\end{gathered}
$$

where $S$ runs over all the neutral scalar fields.

\section{Benchmark}

We show the values of parameters at a benchmark point whose input parameters are given by

$$
D_{Q_{L, R}} \sim D_{\ell_{L, R}} \sim 5 \mathrm{TeV}, \quad m_{N}=10 \mathrm{TeV}, \quad \delta_{u}=\delta_{d}=10^{-4} v_{H},
$$

and the angles for $V_{Q_{L, R}}, V_{\ell_{L, R}}$ are $s_{Q}=0.00078$ and $s_{\ell}=0.00072$. The other unitary matrices in $W_{Q_{L, R}}, W_{\ell_{L, R}}$ and $w_{Q_{L, R}}$, $w_{\ell_{L, R}}$ are taken to be identity. The other parameters are fitted such that the SM fermion masses, CKM and PMNS matrices are explained. The mass matrices in Eq. (2.24) are given by, in unit of $\mathrm{GeV}$,

$$
\tilde{D}_{d}=\left(\begin{array}{cccccc}
0 & 0 & 0 & 0.000502716 & 0 & 0 \\
0 & 0 & 0 & 0 & 0.146787 & 0 \\
0 & 0 & 0 & 0 & 0 . & 1.80177 \\
0.00211529 & 0 & 0 & 0 & 0 & 0 \\
0 & 0.0417809 & 0 & 0 & 0 & 0 \\
0 & 0 & 2.97755 & 0 & 0 & 0
\end{array}\right),
$$


$\tilde{D}_{u}=\left(\begin{array}{cccccc}0.00000060 \times e^{-2.2460 i} & 0.00001279 \times e^{-1.0960 i} & 0.00488531 \times e^{-2.7440 i} & 0.00001386 \times e^{0.0987 i} & 0.00015155 \times e^{0.0092 i} & 0.00013351 \times e^{2.1100 i} \\ 0.00000181 \times e^{-3.1020 i} & 0.00002292 \times e^{-3.1350 i} & 0.00697150 \times e^{-3.0540 i} & 0.00007441 \times e^{3.1210 i} & 0.00003288 \times e^{0.5167 i} & 0.00036822 \times e^{0.0012 i} \\ 0.00000197 \times e^{-3.0910 i} & 0.00006067 \times e^{-3.1380 i} & 0.00863796 \times e^{-3.0440 i} & 0.00006500 \times e^{0.0333 i} & 0.00002488 \times e^{1.1330 i} & 0.00051000 \times e^{0.0012 i} \\ 0.00111051 \times e^{0.0579 i} & 0.10784074 \times e^{3.1410 i} & 1.22353000 \times e^{0.3833 i} & 0.00080864 \times e^{-2.7230 i} & 0.00080780 \times e^{-2.7230 i} & 0.00081043 \times e^{-2.7230 i} \\ 0.00057303 \times e^{3.1160 i} & 0.46812929 & 5.64585730 \times e^{3.1230 i} & 0.00371601 \times e^{-0.0204 i} & 0.00371740 \times e^{-0.0204 i} & 0.00373373 \times e^{-0.0203 i} \\ 0.02694553 \times e^{0.0001 i} & 0.01657569 & 191.73898000 & 0.13766851 \times e^{-3.1420 i} & 0.13769853 \times e^{-3.1420 i} & -0.13788805\end{array}\right)$,

(B12)

$V_{Q_{R}}^{*} \tilde{D}_{Q_{R}}^{T}=\left(\begin{array}{ccc}3.90023160 & 3.90030370 & 3.90037580 \\ 3.90023280 & 3.90030730 & 3.90038170 \\ 3.90023400 & 3.90031080 & 3.90038760 \\ 5000.29540000 & -0.00912672 & -0.00912690 \\ 0.00000000 & 5000.39540000 & -0.00912691 \\ 0.00000000 & 0.00000000 & 5000.49540000\end{array}\right), \quad V_{Q_{L}} \tilde{D}_{Q_{L}}=\left(\begin{array}{cccc}4999.99540000 & -2.82413960 & -0.00818287 \\ 0.00000000 & 3551.78620000 & -2.85575350 \\ 0.00000000 & 0.00000000 & 5000.19540000 \\ -3.89999760 & -2.76819210 & -3.89792210 \\ -3.89999880 & -99.99485400 & -3.82138210 \\ -3.90000000 & -3517.92550000 & -2.77154640\end{array}\right)$,

(B13)

$V_{\ell_{R}}^{*} \tilde{D}_{\ell_{R}}^{T}=\left(\begin{array}{ccc}5000.89610000 & -0.00777755 & -0.00777770 \\ 0.00000000 & 5000.99610000 & -0.00777771 \\ 0.00000000 & 0.00000000 & 5001.09610000 \\ -3.60064610 & -3.60071350 & -3.60078080 \\ -3.60064710 & -3.60071630 & -3.60078550 \\ -3.60064800 & -3.60071910 & -3.60079010\end{array}\right), \quad V_{\ell_{L}} \tilde{D}_{\ell_{L}}=\left(\begin{array}{cccc}3.60043010 & 3.59914710 & 2.48918270 \\ 3.60043110 & -140.73388000 & -3517.02360000 \\ 3.60043200 & 3.60050310 & 3.60057410 \\ 5000.59610000 & 0.09614469 & 2.52787370 \\ 0.00000000 & 4998.71670000 & -99.02272300 \\ 0.00000000 & 0.00000000 & 3553.69030000\end{array}\right)$,

The charged fermion Dirac masses in unit of $\mathrm{GeV}$ are given by

$$
\begin{aligned}
& m_{i}^{e}=(0.00050112,0.105789,1.80177,4999.87,5000.6,5000.7,5000.9,5001,5002.03), \\
& m_{i}^{d}=(0.0021141,0.04176,2.11584,4999.43,5000,5000.09,5000.3,5000.41,5001.27), \\
& m_{i}^{u}=(0.0009744,0.479892,136.433,5000,5000.2,5000.3,5000.4,5000.5,5001.92) .
\end{aligned}
$$

The neutral fermion Majorana masses in unit of $\mathrm{GeV}$ are given by

$$
\begin{aligned}
m_{i}^{n}= & \left(3.38664 \times 10^{-13}, 8.68414 \times 10^{-12}, 5.01938 \times 10^{-11},\right. \\
& 4934.06,4934.06,5000.55,5000.55,5000.61,5000.61,5000.89,5000.9, \\
& 5001.13,5001.14,5070.58,5070.59,10000,10000,10000) .
\end{aligned}
$$

The $W$-boson couplings are proportional to

$\left(U_{L}^{u}\right)^{\dagger} P_{6} U_{L}^{d}=\left(\begin{array}{ccccccccc}0.9745 & 0.2245 & 0.0036 \times e^{-1.20 i} & 0.0000 & 0.0000 \times e^{-0.49 i} & 0.0000 \times e^{-0.30 i} & 0.0000 \times e^{-0.43 i} & 0.0000 \times e^{2.80 i} & 0.0000 \times e^{-3.10 i} \\ 0.2244 \times e^{-3.10 i} & 0.9736 & 0.0421 & 0.0000 & 0.0000 & 0.0000 & 0.0000 & 0.0000 \times e^{-3.10 i} & 0.0000 \times e^{-3.10 i} \\ 0.0090 \times e^{-0.38 i} & 0.0413 \times e^{-3.10 i} & 0.9991 & 0.0000 \times e^{0.02 i} & 0.0000 \times e^{3.10 i} & 0.0007 \times e^{-3.10 i} & 0.0000 \times e^{-3.10 i} & 0.0002 \times e^{-3.10 i} & -0.0000 \\ 0.0000 \times e^{-0.42 i} & 0.0000 \times e^{-3.10 i} & 0.0000 & 0.0000 \times e^{1.50 i} & 0.9991 & 0.0007 \times e^{-3.10 i} & 0.0298 \times e^{-3.10 i} & 0.0002 \times e^{-3.10 i} & 0.0000 \times e^{1.40 i} \\ 0.0000 \times e^{-2.80 i} & 0.0000 \times e^{0.73 i} & 0.0000 \times e^{-2.40 i} & 0.7621 \times e^{0.72 i} & 0.0000 \times e^{3.00 i} & 0.0000 & 0.0000 \times e^{-0.33 i} & 0.0000 \times e^{-2.30 i} & 0.6468 \times e^{0.72 i} \\ 0.0000 \times e^{-0.22 i} & 0.0000 \times e^{-3.10 i} & 0.0000 & 0.0002 \times e^{-0.03 i} & 0.0290 & 0.0025 & 0.0009 \times e^{-3.10 i} & 0.0005 & 0.0002 \times e^{-0.03 i} \\ 0.0000 \times e^{-0.41 i} & 0.0000 \times e^{-3.10 i} & 0.0000 \times e^{-0.01 i} & 0.0000 \times e^{1.00 i} & 0.0002 \times e^{-3.10 i} & 0.0029 & 0.0000 & 0.0006 & 0.0000 \times e^{1.00 i} \\ 0.0000 \times e^{-1.30 i} & 0.0000 \times e^{2.10 i} & 0.0000 \times e^{-1.00 i} & 0.0227 \times e^{-1.00 i} & 0.0001 \times e^{-3.10 i} & 0.0028 \times e^{-1.00 i} & 0.0000 \times e^{-0.73 i} & 0.0005 \times e^{-1.00 i} & 0.0193 \times e^{-1.00 i} \\ 0.0000 \times e^{-0.36 i} & 0.0000 \times e^{-3.10 i} & 0.0007 \times e^{0.03 i} & 0.0001 \times e^{-3.10 i} & 0.0008 \times e^{0.01 i} & 0.9819 \times e^{0.03 i} & 0.0034 \times e^{0.03 i} & 0.1894 \times e^{0.03 i} & 0.0000 \times e^{-0.26 i}\end{array}\right)$, 
$\left(U_{L}^{n}\right)^{\dagger} P_{6} U_{L}^{e}$

\begin{tabular}{|c|c|c|c|c|c|c|c|c|}
\hline $0.7900 \times e^{-0.83 i}$ & $0.3877 \times e^{2.40 i}$ & $0.4748 \times e^{-0.41 i}$ & $0.0001 \times e^{2.40 i}$ & $0.0000 \times e^{-0.38 i}$ & $0.0000 \times e^{-0.59 i}$ & $0.0000 \times e^{2.80 i}$ & $0.0000 \times e^{-0.59 i}$ & $0.0001 \times e^{-0.77 i}$ \\
\hline $0.5949 \times e^{1.30 i}$ & $0.5746 \times e^{1.70 i}$ & $0.5619 \times e^{-1.70 i}$ & $0.0002 \times e^{1.70 i}$ & $0.0000 \times e^{-1.70 i}$ & $0.0000 \times e^{-1.60 i}$ & $0.0000 \times e^{1.40 i}$ & $0.0000 \times e^{-1.60 i}$ & $0.0002 \times e^{-1.50 i}$ \\
\hline $0.1482 \times e^{0.13 i}$ & $0.7202 \times e^{-1.60 i}$ & $0.6774 \times e^{-1.60 i}$ & $0.0002 \times e^{-1.60 i}$ & $0.0000 \times e^{-1.60 i}$ & $0.0000 \times e^{1.60 i}$ & $0.0000 \times e^{1.60 i}$ & $0.0000 \times e^{1.60 i}$ & $0.0002 \times e^{1.60 i}$ \\
\hline $0.0000 \times e^{-3.10 i}$ & 0.0135 & $0.0000 \times e^{-0.12 i}$ & 0.3774 & $0.0045 \times e^{-2.80 i}$ & $0.0206 \times e^{-0.02 i}$ & $0.0001 \times e^{-2.80 i}$ & $0.0020 \times e^{3.10 i}$ & 0.3280 \\
\hline $0.0000 \times e^{-1.60 i}$ & $0.0135 \times e^{1.60 i}$ & $0.0000 \times e^{1.40 i}$ & $0.3774 \times e^{1.60 i}$ & $0.0045 \times e^{-1.20 i}$ & $0.0206 \times e^{1.60 i}$ & $0.0001 \times e^{-1.20 i}$ & $0.0020 \times e^{-1.60 i}$ & $0.3280 \times e^{1.60 i}$ \\
\hline $0.0000 \times e^{-3.10 i}$ & $0.0000 \times e^{-0.02 i}$ & $0.0000 \times e^{-3.10 i}$ & $0.0185 \times e^{-0.03 i}$ & 0.3424 & $0.5213 \times e^{-3.10 i}$ & 0.0111 & 0.0509 & $0.0161 \times e^{-0.03 i}$ \\
\hline $0.0000 \times e^{-1.50 i}$ & $0.0000 \times e^{1.60 i}$ & $0.0000 \times e^{-1.60 i}$ & $0.0185 \times e^{1.50 i}$ & $0.3473 \times e^{1.60 i}$ & $0.5200 \times e^{-1.60 i}$ & $0.0113 \times e^{1.60 i}$ & $0.0508 \times e^{1.60 i}$ & $0.0161 \times e^{1.50 i}$ \\
\hline $0.0000 \times e^{-3.10 i}$ & $0.0000 \times e^{0.16 i}$ & -0.0000 & $0.0065 \times e^{0.27 i}$ & -0.6148 & -0.3247 & -0.0200 & 0.0317 & $0.0057 \times e^{0.27 i}$ \\
\hline $0.0000 \times e^{-1.50 i}$ & $0.0000 \times e^{1.70 i}$ & $0.0000 \times e^{-1.60 i}$ & $0.0067 \times e^{1.80 i}$ & $0.6120 \times e^{-1.60 i}$ & $0.3294 \times e^{-1.60 i}$ & $0.0199 \times e^{-1.60 i}$ & $0.0322 \times e^{1.60 i}$ & $0.0058 \times e^{1.80 i}$ \\
\hline $0.0000 \times e^{0.06 i}$ & $0.0000 \times e^{-3.10 i}$ & 0.0000 & $0.0006 \times e^{0.10 i}$ & $0.0192 \times e^{-3.10 i}$ & $0.0203 \times e^{-3.10 i}$ & $0.0006 \times e^{-3.10 i}$ & 0.0020 & $0.0005 \times e^{0.10 i}$ \\
\hline $0.0000 \times e^{1.70 i}$ & $0.0000 \times e^{-1.50 i}$ & $0.0000 \times e^{1.60 i}$ & $0.0002 \times e^{1.90 i}$ & $0.0205 \times e^{-1.60 i}$ & $0.0091 \times e^{-1.60 i}$ & $0.0007 \times e^{-1.60 i}$ & $0.0009 \times e^{1.60 i}$ & $0.0002 \times e^{1.90 i}$ \\
\hline $0.0000 \times e^{0.04 i}$ & $0.0000 \times e^{-3.10 i}$ & 0.0000 & 0.0112 & 0.0624 & $0.3418 \times e^{-3.10 i}$ & 0.0020 & 0.0334 & 0.0097 \\
\hline $0.0000 \times e^{1.60 i}$ & $0.0000 \times e^{-1.60 i}$ & $0.0000 \times e^{1.60 i}$ & $0.0111 \times e^{1.60 i}$ & $0.0619 \times e^{1.60 i}$ & $0.3397 \times e^{-1.60 i}$ & $0.0020 \times e^{1.60 i}$ & $0.0332 \times e^{1.60 i}$ & $0.0096 \times e^{1.60 i}$ \\
\hline $0.0000 \times e^{-3.10 i}$ & 0.0135 & $0.0000 \times e^{-0.12 i}$ & -0.3767 & $0.0045 \times e^{0.38 i}$ & $0.0205 \times e^{3.10 i}$ & $0.0001 \times e^{0.38 i}$ & $0.0020 \times e^{-0.02 i}$ & -0.3274 \\
\hline $0.0000 \times e^{-1.60 i}$ & $0.0135 \times e^{1.60 i}$ & $0.0000 \times e^{1.40 i}$ & $0.3767 \times e^{-1.60 i}$ & $0.0045 \times e^{2.00 i}$ & $0.0205 \times e^{-1.60 i}$ & $0.0001 \times e^{1.90 i}$ & $0.0020 \times e^{1.60 i}$ & $0.3274 \times e^{-1.60 i}$ \\
\hline $0.0000 \times e^{1.10 i}$ & $0.0000 \times e^{-0.22 i}$ & $0.0000 \times e^{-0.56 i}$ & $0.0000 \times e^{-0.32 i}$ & $0.0000 \times e^{1.90 i}$ & $0.0000 \times e^{-0.76 i}$ & $0.0000 \times e^{2.00 i}$ & $0.0000 \times e^{2.40 i}$ & $0.0000 \times e^{-0.32 i}$ \\
\hline $0.0000 \times e^{2.70 i}$ & $0.0000 \times e^{-0.40 i}$ & $0.0000 \times e^{-0.17 i}$ & $0.0000 \times e^{-0.27 i}$ & $0.0000 \times e^{-3.00 i}$ & $0.0000 \times e^{-0.04 i}$ & $0.0000 \times e^{-3.00 i}$ & $0.0000 \times e^{3.10 i}$ & $0.0000 \times e^{-0.27 i}$ \\
\hline $0.0000 \times e^{0.23 i}$ & -0.0000 & $0.0000 \times e^{0.05 i}$ & 0.0000 & $0.0000 \times e^{-2.70 i}$ & $0.0000 \times e^{-0.02 i}$ & $0.0000 \times e^{-2.70 i}$ & $0.0000 \times e^{3.10 i}$ & 0.0000 \\
\hline
\end{tabular}

$\left(U_{R}^{u}\right)^{\dagger} P_{\overline{6}} U_{R}^{d}$

$=\left(\begin{array}{ccccccccc}0.0000 \times e^{-0.11 i} & 0.0000 \times e^{-0.23 i} & 0.0000 \times e^{-0.26 i} & 0.0000 \times e^{-3.10 i} & 0.0000 \times e^{2.80 i} & 0.0000 \times e^{2.90 i} & 0.0000 \times e^{3.00 i} & 0.0000 \times e^{2.90 i} & 0.0000 \times e^{-3.10 i} \\ 0.0000 & 0.0000 & 0.0000 & 0.0000 \times e^{-3.10 i} & 0.0000 \times e^{3.10 i} & 0.0000 \times e^{-3.10 i} & 0.0000 \times e^{-3.10 i} & 0.0000 \times e^{-3.10 i} & 0.0000 \times e^{-3.10 i} \\ 0.0000 & 0.0000 & 0.0000 & 0.0000 \times e^{0.05 i} & 0.0000 \times e^{3.10 i} & 0.0265 \times e^{-3.10 i} & 0.0001 \times e^{-3.10 i} & 0.0051 \times e^{-3.10 i} & 0.0000 \times e^{1.50 i} \\ -0.0000 & -0.0000 & 0.0000 \times e^{0.90 i} & 0.0000 \times e^{1.50 i} & 0.9991 & 0.0007 \times e^{-3.10 i} & 0.0298 \times e^{-3.10 i} & 0.0002 \times e^{-3.10 i} & 0.0000 \times e^{1.40 i} \\ 0.0000 \times e^{-2.40 i} & 0.0000 \times e^{-2.40 i} & 0.0000 \times e^{-2.40 i} & 0.7622 \times e^{0.72 i} & 0.0000 \times e^{3.00 i} & 0.0000 & 0.0000 \times e^{-0.33 i} & 0.0000 \times e^{-2.30 i} & 0.6467 \times e^{0.72 i} \\ 0.0000 \times e^{3.10 i} & -0.0000 & -0.0000 & 0.0002 \times e^{-0.03 i} & 0.0290 & 0.0025 & 0.0009 \times e^{-3.10 i} & 0.0005 & 0.0002 \times e^{-0.03 i} \\ 0.0000 \times e^{-3.00 i} & 0.0000 \times e^{-3.10 i} & 0.0000 \times e^{3.10 i} & 0.0000 \times e^{1.00 i} & 0.0002 \times e^{-3.10 i} & 0.0029 & 0.0000 & 0.0006 & 0.0000 \times e^{1.00 i} \\ 0.0000 \times e^{2.10 i} & 0.0000 \times e^{2.10 i} & 0.0000 \times e^{2.10 i} & 0.0227 \times e^{-1.00 i} & 0.0001 \times e^{-3.10 i} & 0.0028 \times e^{-1.00 i} & 0.0000 \times e^{-0.73 i} & 0.0005 \times e^{-1.00 i} & 0.0193 \times e^{-1.00 i} \\ 0.0000 \times e^{-3.10 i} & 0.0000 \times e^{-3.10 i} & 0.0004 \times e^{-3.10 i} & 0.0001 \times e^{-3.10 i} & 0.0008 \times e^{0.01 i} & 0.9815 \times e^{0.03 i} & 0.0034 \times e^{0.03 i} & 0.1893 \times e^{0.03 i} & 0.0000 \times e^{-0.26 i}\end{array}\right)$,

(B19) 
$\left(U_{R}^{n}\right)^{\dagger} P_{\overline{6}} U_{R}^{e}$

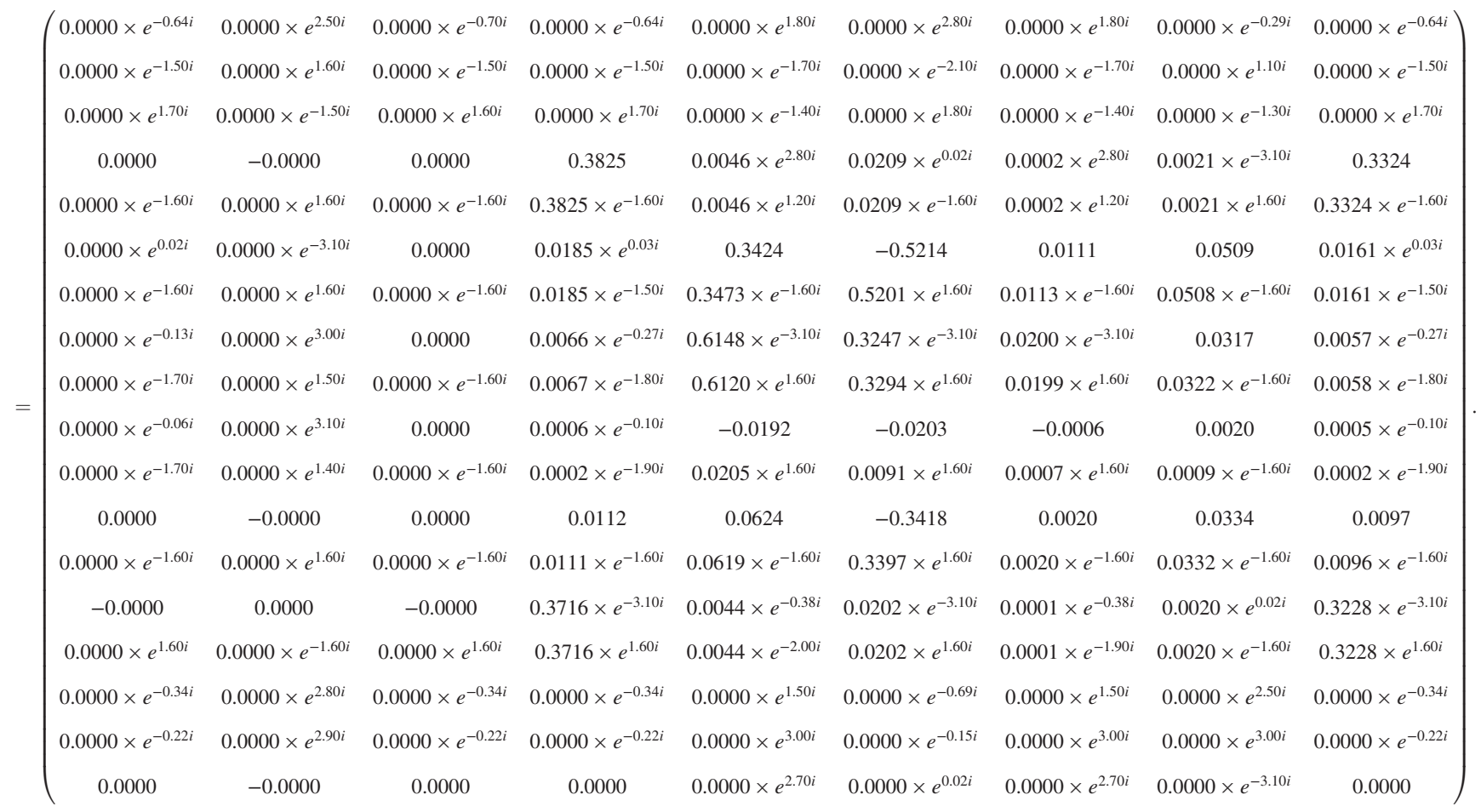

(B20)

The upper-left $3 \times 3$ block of $U_{u_{L}}^{\dagger} P_{6} U_{d_{L}}$ and $U_{n_{L}}^{\dagger} P_{6} U_{e_{L}}$ corresponds to the CKM matrix and Hermitian conjugate of the PMNS matrix, respectively. We also note that the $W$-boson couplings to the SM fermions in the right-handed current are negligible. The leptoquark couplings with $g_{4}=1$ are given by

$\hat{g}_{d_{L}}^{X}=\left(\begin{array}{cccccccccc}0.0000 & 0.0000 & 0.0000 & 0.0000 & 0.7067 & 0.0000 & 0.0230 & 0.0000 \times e^{-3.10 i} & 0.0000 \\ 0.0000 & 0.0283 & 0.0001 & 0.0211 & 0.0000 \times e^{-3.10 i} & 0.7026 & 0.0000 & 0.0686 \times e^{-3.10 i} & 0.0184 \\ 0.0000 \times e^{-3.10 i} & 0.7065 & 0.0012 & 0.0055 \times e^{-3.10 i} & 0.0000 \times e^{-3.10 i} & 0.0279 \times e^{-3.10 i} & 0.0000 \times e^{-3.10 i} & 0.0027 & 0.0052 \times e^{-3.10 i} \\ 0.0000 & 0.0007 & -0.5391 & 0.3009 & 0.0000 & -0.0000 & -0.0000 & -0.0001 & -0.3447 \\ -0.7068 & -0.0000 & 0.0000 & 0.0001 & -0.0006 & 0.0000 & 0.0211 & 0.0000 & 0.0001 \\ -0.0002 & -0.0064 & -0.0012 & -0.5235 & 0.0001 & 0.0146 & -0.0027 & -0.1360 & -0.4551 \\ 0.0211 & -0.0000 & -0.0000 & -0.0018 & -0.0230 & -0.0001 & 0.7064 & -0.0015 & -0.0016 \\ 0.0000 & -0.0012 & -0.0002 & -0.1009 & -0.0000 & 0.0728 & 0.0010 & 0.6905 & -0.0879 \\ -0.0000 & 0.0010 & -0.4576 & -0.3531 & 0.0000 & 0.0000 & 0.0000 & 0.0001 & 0.4074\end{array}\right)$

$\hat{g}_{d_{R}}^{X}=\left(\begin{array}{ccccccccc}0.0000 \times e^{-3.10 i} & 0.0000 \times e^{-3.10 i} & 0.0000 \times e^{-3.10 i} & 0.0000 & 0.0230 \times e^{-3.10 i} & 0.0000 \times e^{-3.10 i} & 0.7067 & 0.0001 \times e^{-3.10 i} & 0.0000 \times e^{-3.10 i} \\ 0.0000 \times e^{-3.10 i} & 0.0000 \times e^{-3.10 i} & 0.0000 \times e^{-3.10 i} & 0.0000 & 0.0000 & 0.0687 \times e^{-3.10 i} & 0.0001 \times e^{-3.10 i} & 0.7038 \times e^{-3.10 i} & 0.0001 \times e^{-3.10 i} \\ 0.0000 \times e^{-3.10 i} & 0.0000 \times e^{-3.10 i} & 0.0000 \times e^{-3.10 i} & 0.4638 & 0.0000 & 0.0003 & 0.0001 \times e^{-3.10 i} & 0.0000 & 0.5338 \times e^{-3.10 i} \\ 0.0000 & -0.0001 & 0.4575 & 0.4070 & -0.0000 & 0.0006 & 0.0000 & -0.0001 & 0.3536 \\ 0.0211 & 0.0003 & 0.0000 & 0.0000 & -0.7064 & 0.0002 & -0.0230 & -0.0000 & -0.0000 \\ -0.0046 & 0.1339 & 0.0001 & 0.0007 & -0.0003 & -0.6910 & -0.0000 & 0.0675 & 0.0002 \\ 0.7067 & 0.0116 & 0.0000 & 0.0000 & 0.0211 & -0.0024 & 0.0007 & 0.0002 & -0.0000 \\ 0.0109 & -0.6942 & -0.0001 & 0.0001 & 0.0000 & -0.1333 & -0.0000 & 0.0131 & 0.0001 \\ -0.0000 & 0.0001 & -0.5392 & 0.3453 & 0.0000 & 0.0004 & -0.0000 & -0.0000 & 0.3001\end{array}\right)$

(B22) 
$\left(\hat{g}_{u_{L}}^{X}\right)^{\dagger}$

\begin{tabular}{|c|c|c|c|c|c|c|c|c|}
\hline $0.0029 \times e^{-2.00 i}$ & $0.0221 \times e^{-2.40 i}$ & $0.2727 \times e^{-2.40 i}$ & $0.5584 \times e^{-2.30 i}$ & $0.3358 \times e^{2.80 i}$ & $0.0163 \times e^{-2.30 i}$ & $0.0001 \times e^{1.10 i}$ & $0.0171 \times e^{-1.60 i}$ & $0.0013 \times e^{0.84 i}$ \\
\hline $0.0044 \times e^{-1.40 i}$ & $0.0329 \times e^{-1.70 i}$ & $0.4043 \times e^{-1.70 i}$ & $0.4204 \times e^{1.80 i}$ & $0.3975 \times e^{-2.20 i}$ & $0.0121 \times e^{1.80 i}$ & $0.0001 \times e^{-1.60 i}$ & $0.0227 \times e^{-0.56 i}$ & $0.0032 \times e^{1.40 i}$ \\
\hline $0.0055 \times e^{1.90 i}$ & $0.0414 \times e^{1.50 i}$ & $0.5083 \times e^{1.50 i}$ & $0.1048 \times e^{3.00 i}$ & $0.4784 \times e^{-2.30 i}$ & $0.0030 \times e^{3.10 i}$ & $0.0000 \times e^{-1.90 i}$ & $0.0006 \times e^{-0.25 i}$ & $0.0051 \times e^{-1.60 i}$ \\
\hline $0.0039 \times e^{0.32 i}$ & 0.0291 & 0.0042 & $0.0004 \times e^{-0.12 i}$ & $0.0111 \times e^{-0.72 i}$ & $0.0010 \times e^{-3.10 i}$ & $0.0011 \times e^{-3.10 i}$ & $0.3539 \times e^{-2.10 i}$ & $0.3518 \times e^{3.10 i}$ \\
\hline $0.0039 \times e^{-1.30 i}$ & $0.0291 \times e^{-1.60 i}$ & $0.0042 \times e^{-1.60 i}$ & $0.0004 \times e^{-1.70 i}$ & $0.0111 \times e^{-2.30 i}$ & $0.0010 \times e^{1.60 i}$ & $0.0011 \times e^{1.60 i}$ & $0.3539 \times e^{2.60 i}$ & $0.3518 \times e^{1.50 i}$ \\
\hline 0.1532 & -0.4131 & $0.0318 \times e^{0.01 i}$ & 0.0000 & $0.0000 \times e^{-0.99 i}$ & 0.0010 & 0.2319 & $0.0002 \times e^{-2.10 i}$ & $0.0328 \times e^{3.10 i}$ \\
\hline $0.1567 \times e^{-1.60 i}$ & $0.4130 \times e^{1.60 i}$ & $0.0317 \times e^{-1.60 i}$ & $0.0001 \times e^{1.60 i}$ & $0.0000 \times e^{-2.60 i}$ & $0.0046 \times e^{-1.60 i}$ & $0.2297 \times e^{-1.60 i}$ & $0.0002 \times e^{2.60 i}$ & $0.0327 \times e^{1.60 i}$ \\
\hline-0.4755 & -0.1260 & $0.0151 \times e^{-0.11 i}$ & 0.0005 & $0.0000 \times e^{3.00 i}$ & $0.0166 \times e^{-3.10 i}$ & 0.0855 & $0.0001 \times e^{-2.30 i}$ & $0.0154 \times e^{3.00 i}$ \\
\hline $0.4743 \times e^{1.60 i}$ & $0.1297 \times e^{1.60 i}$ & $0.0154 \times e^{-1.70 i}$ & $0.0004 \times e^{-1.60 i}$ & $0.0000 \times e^{1.50 i}$ & $0.0147 \times e^{1.60 i}$ & $0.0865 \times e^{-1.60 i}$ & $0.0001 \times e^{2.40 i}$ & $0.0157 \times e^{1.40 i}$ \\
\hline-0.0165 & -0.0109 & $0.0010 \times e^{-0.06 i}$ & $0.0145 \times e^{-3.10 i}$ & $0.0001 \times e^{2.40 i}$ & 0.4993 & $0.0113 \times e^{-3.10 i}$ & $0.0001 \times e^{-2.20 i}$ & $0.0024 \times e^{3.10 i}$ \\
\hline $0.0156 \times e^{1.60 i}$ & $0.0030 \times e^{1.60 i}$ & $0.0004 \times e^{-1.70 i}$ & $0.0145 \times e^{1.60 i}$ & $0.0001 \times e^{0.82 i}$ & $0.4995 \times e^{-1.60 i}$ & $0.0051 \times e^{1.60 i}$ & $0.0001 \times e^{2.50 i}$ & $0.0017 \times e^{1.50 i}$ \\
\hline $0.0113 \times e^{3.10 i}$ & -0.2451 & 0.0199 & 0.0004 & $0.0000 \times e^{-0.17 i}$ & $0.0157 \times e^{-3.10 i}$ & $0.4345 \times e^{-3.10 i}$ & $0.0000 \times e^{0.97 i}$ & $0.0188 \times e^{3.10 i}$ \\
\hline $0.0114 \times e^{1.60 i}$ & $0.2436 \times e^{1.60 i}$ & $0.0198 \times e^{-1.60 i}$ & $0.0001 \times e^{-1.60 i}$ & $0.0000 \times e^{-1.70 i}$ & $0.0064 \times e^{1.60 i}$ & $0.4356 \times e^{1.60 i}$ & $0.0000 \times e^{-0.60 i}$ & $0.0187 \times e^{1.50 i}$ \\
\hline $0.0037 \times e^{-2.80 i}$ & -0.0275 & 0.0148 & $0.0004 \times e^{-3.10 i}$ & $0.0100 \times e^{-0.72 i}$ & 0.0009 & 0.0009 & $0.3526 \times e^{-2.10 i}$ & $0.3530 \times e^{-0.03 i}$ \\
\hline $0.0037 \times e^{1.90 i}$ & $0.0275 \times e^{1.60 i}$ & $0.0148 \times e^{-1.60 i}$ & $0.0004 \times e^{1.70 i}$ & $0.0100 \times e^{-2.30 i}$ & $0.0009 \times e^{-1.60 i}$ & $0.0009 \times e^{-1.60 i}$ & $0.3526 \times e^{2.60 i}$ & $0.3529 \times e^{-1.60 i}$ \\
\hline $0.0000 \times e^{0.36 i}$ & $0.0000 \times e^{0.34 i}$ & $0.0000 \times e^{0.21 i}$ & $0.0000 \times e^{2.00 i}$ & $0.0000 \times e^{3.00 i}$ & $0.0000 \times e^{3.00 i}$ & $0.0000 \times e^{0.39 i}$ & $0.0000 \times e^{-1.40 i}$ & $0.0000 \times e^{-2.90 i}$ \\
\hline $0.0000 \times e^{0.53 i}$ & $0.0000 \times e^{0.24 i}$ & $0.0000 \times e^{0.40 i}$ & $0.0000 \times e^{0.48 i}$ & $0.0000 \times e^{2.60 i}$ & $0.0000 \times e^{3.10 i}$ & $0.0000 \times e^{0.21 i}$ & $0.0000 \times e^{-2.00 i}$ & $0.0000 \times e^{-2.90 i}$ \\
\hline $0.0000 \times e^{0.32 i}$ & 0.0000 & -0.0000 & -0.0000 & $0.0000 \times e^{2.40 i}$ & 0.0006 & $0.0006 \times e^{-3.10 i}$ & $0.0006 \times e^{1.00 i}$ & $0.0000 \times e^{3.10 i}$ \\
\hline
\end{tabular}

$\left(\hat{g}_{u_{R}}^{X}\right)^{\dagger}$

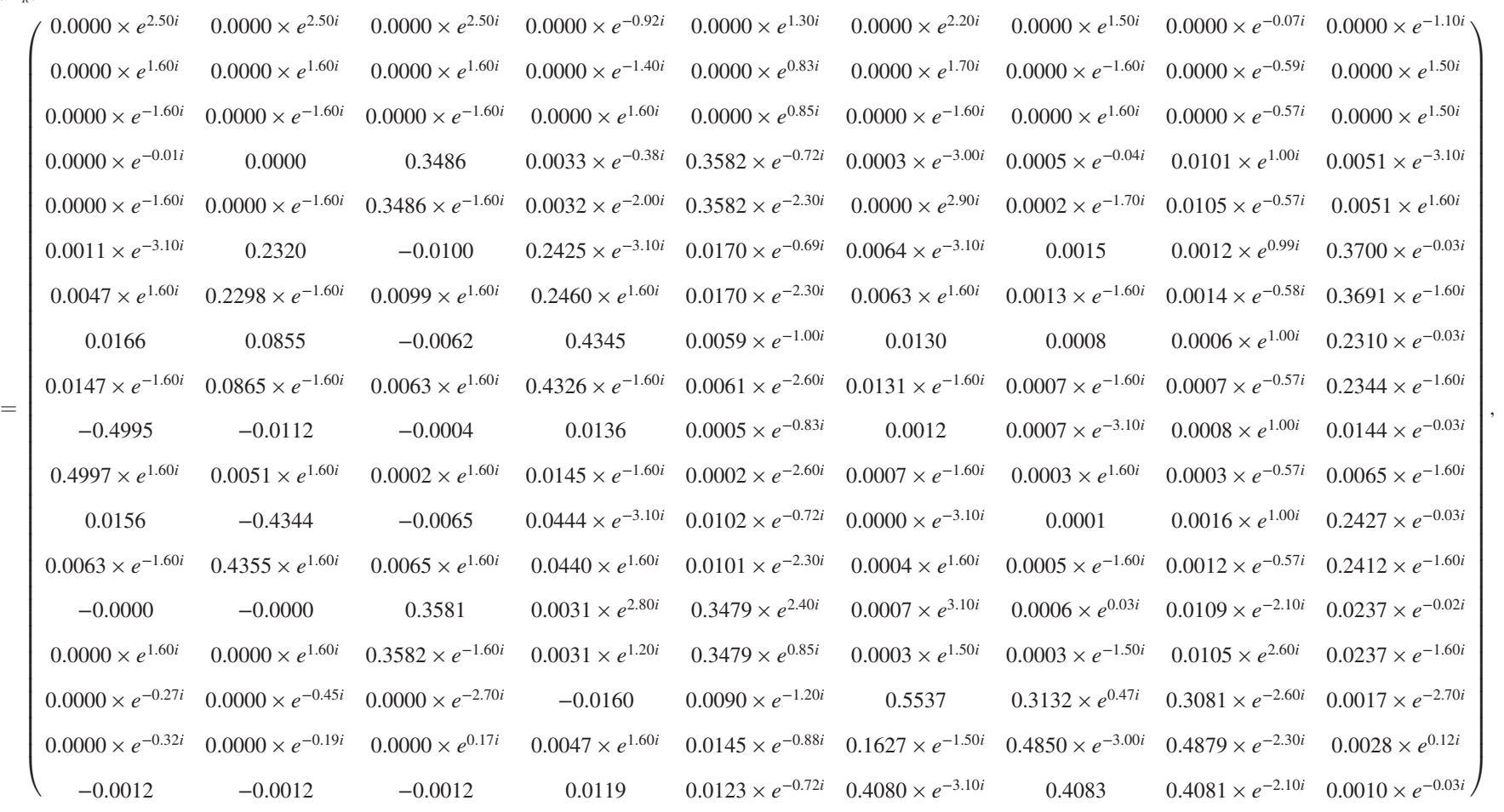

The Yukawa couplings $h_{q q^{\prime}}$ defined in Eq. (3.38) are given by

$$
h_{d d}=0.00000183 \times e^{-0.01 i}, \quad h_{u и}=0.00000182 \times e^{-0.01 i}, \quad h_{d u}=0.00000183 \times e^{-0.01 i},
$$

where the Yukawa coupling in the gauge basis, $h$ is set to be identity matrix. 
TABLE IV. The values of predictions at the benchmark point, lower and upper limit are shown. The value of the fine-tuning parameter is $\Delta_{\mathrm{FT}}^{-1}=1.39 \times 10^{3}$.

\begin{tabular}{|c|c|c|c|c|}
\hline Observable & Prediction & Lower limit & Upper limit & Ref. \\
\hline $\operatorname{Re} \Delta C_{9}(b \rightarrow s \mu \mu$ anomaly $)$ & -0.505 & -0.59 & -0.41 & [89] \\
\hline$\Delta C_{V}(b \rightarrow c \tau \nu$ anomaly $)$ & $2.75 \times 10^{-8}$ & $5.2 \times 10^{-2}$ & 0.124 & [90] \\
\hline $\mathrm{BR}(B \rightarrow e \nu)$ & $8.74 \times 10^{-12}$ & 0 & $9.8 \times 10^{-7}$ & [95] \\
\hline $\mathrm{BR}(B \rightarrow \mu \nu)$ & $3.88 \times 10^{-7}$ & $2.90 \times 10^{-7}$ & $1.07 \times 10^{-6}$ & [95] \\
\hline $\mathrm{BR}(B \rightarrow \tau \nu)$ & $8.82 \times 10^{-5}$ & $8.5 \times 10^{-5}$ & $1.33 \times 10^{-4}$ & {$[95]$} \\
\hline $\mathrm{BR}\left(B_{c} \rightarrow e \nu\right)$ & $2.20 \times 10^{-9}$ & 0 & 0.6 & {$[117]$} \\
\hline $\mathrm{BR}\left(B_{c} \rightarrow \mu \nu\right)$ & $9.79 \times 10^{-5}$ & 0 & 0.6 & {$[117]$} \\
\hline $\mathrm{BR}\left(B_{c} \rightarrow \tau \nu\right)$ & $2.39 \times 10^{-2}$ & 0 & 0.6 & [117] \\
\hline $\mathrm{BR}\left(K_{L} \rightarrow e \mu\right)$ & $3.04 \times 10^{-13}$ & 0 & $4.7 \times 10^{-12}$ & [95] \\
\hline $\mathrm{BR}\left(B_{d} \rightarrow e \tau\right)$ & $1.27 \times 10^{-18}$ & 0 & $2.8 \times 10^{-5}$ & [95] \\
\hline $\mathrm{BR}\left(B_{d} \rightarrow \mu \tau\right)$ & $4.70 \times 10^{-13}$ & 0 & $2.2 \times 10^{-5}$ & [95] \\
\hline $\mathrm{BR}\left(B_{d} \rightarrow \mu e\right)$ & $5.46 \times 10^{-13}$ & 0 & $2.8 \times 10^{-9}$ & [95] \\
\hline $\mathrm{BR}\left(B_{d} \rightarrow e e\right)$ & $2.47 \times 10^{-15}$ & 0 & $8.3 \times 10^{-8}$ & [95] \\
\hline $\mathrm{BR}\left(B_{d} \rightarrow \mu \mu\right)$ & $1.00 \times 10^{-10}$ & 0 & $3.9 \times 10^{-10}$ & [95] \\
\hline $\mathrm{BR}\left(B_{d} \rightarrow \tau \tau\right)$ & $2.34 \times 10^{-8}$ & 0 & $2.1 \times 10^{-3}$ & [95] \\
\hline $\mathrm{BR}\left(B_{s} \rightarrow e \tau\right)$ & $2.83 \times 10^{-18}$ & $\ldots$ & $\ldots$ & [95] \\
\hline $\mathrm{BR}\left(B_{s} \rightarrow \mu \tau\right)$ & $7.51 \times 10^{-13}$ & 0 & $4.2 \times 10^{-5}$ & [95] \\
\hline $\mathrm{BR}\left(B_{s} \rightarrow \mu e\right)$ & $7.99 \times 10^{-13}$ & 0 & $5.4 \times 10^{-9}$ & [95] \\
\hline $\mathrm{BR}\left(B_{s} \rightarrow e e\right)$ & $7.48 \times 10^{-14}$ & 0 & $2.8 \times 10^{-7}$ & [95] \\
\hline $\mathrm{BR}\left(B_{s} \rightarrow \mu \mu\right)$ & $3.81 \times 10^{-9}$ & $2.2 \times 10^{-9}$ & $3.8 \times 10^{-9}$ & [95] \\
\hline $\mathrm{BR}\left(B_{s} \rightarrow \tau \tau\right)$ & $7.16 \times 10^{-7}$ & 0 & $6.8 \times 10^{-3}$ & [95] \\
\hline $\mathrm{BR}(\mu \rightarrow e)^{\mathrm{Al}}$ & $7.67 \times 10^{-16}$ & 0 & $6 \times 10^{-17}$ & [96] \\
\hline $\mathrm{BR}(\mu \rightarrow e)^{\mathrm{Au}}$ & $9.37 \times 10^{-16}$ & 0 & $3 \times 10^{-13}$ & [98](prospect) \\
\hline$\left|C_{B_{d}}\right|$ & 0.94 & 0.83 & 1.27 & {$[105,106]$} \\
\hline $\operatorname{Arg} C_{B_{d}}$ & -0.75 & -5.6 & 1.6 & {$[105,106]$} \\
\hline$\left|C_{B_{s}}\right|$ & 1.03 & 0.93 & 1.29 & {$[105,106]$} \\
\hline $\operatorname{Arg} C_{B_{s}}$ & $-1.71 \times 10^{-2}$ & -1.36 & 2.2 & {$[105,106]$} \\
\hline $\operatorname{Im} C_{K}$ & 0.92 & 0.88 & 1.36 & {$[105,106]$} \\
\hline $\mathrm{BR}(\mu \rightarrow e \gamma)$ & $3.69 \times 10^{-14}$ & 0 & $4.2 \times 10^{-13}$ & {$[101]$} \\
\hline $\mathrm{BR}(\tau \rightarrow e \gamma)$ & $4.38 \times 10^{-15}$ & 0 & $3.3 \times 10^{-8}$ & [95] \\
\hline $\operatorname{BR}(\tau \rightarrow \mu \gamma)$ & $5.09 \times 10^{-9}$ & 0 & $4.4 \times 10^{-8}$ & [95] \\
\hline
\end{tabular}

Finally we summarize the prediction of observables at this benchmark point, lower and upper limits in Table IV. We take $\tan \beta=50$ and $m_{H}=3.0 \mathrm{TeV}$ to calculate the neutral meson mixing. The benchmark point explains the $b \rightarrow s \mu \mu$ anomaly. On the other hand, it is hard to explain the $b \rightarrow c \tau \nu$ anomaly. All experimental constraints listed in the table are satisfied and the tuning level defined in Eq. (2.41) is about $0.1 \%$.
[1] W. Buchmuller, R. Ruckl, and D. Wyler, Leptoquarks in lepton-quark collisions, Phys. Lett. B 191, 442 (1987); Erratum, Phys. Lett. B 448, 320 (1999).

[2] Y. Sakaki, M. Tanaka, A. Tayduganov, and R. Watanabe, Testing leptoquark models in $\bar{B} \rightarrow D^{(*)} \tau \bar{\nu}$, Phys. Rev. D 88, 094012 (2013).

[3] M. Bauer and M. Neubert, Minimal Leptoquark Explanation for the $\mathrm{R}_{D^{(*)}}, \mathrm{R}_{K}$, and $(g-2)_{g}$ Anomalies, Phys. Rev. Lett. 116, 141802 (2016).
[4] S. Fajfer and N. Košnik, Vector leptoquark resolution of $R_{K}$ and $R_{D^{(*)}}$ puzzles, Phys. Lett. B 755, 270 (2016).

[5] D. Bečirević, S. Fajfer, N. Košnik, and O. Sumensari, Leptoquark model to explain the $B$-physics anomalies, $R_{K}$ and $R_{D}$, Phys. Rev. D 94, 115021 (2016).

[6] B. Bhattacharya, A. Datta, J.-P. Guévin, D. London, and R. Watanabe, Simultaneous explanation of the $R_{K}$ and $R_{D^{(*)}}$ puzzles: A model analysis, J. High Energy Phys. 01 (2017) 015 . 
[7] X.-Q. Li, Y.-D. Yang, and X. Zhang, Revisiting the one leptoquark solution to the $R\left(\mathrm{D}^{(*)}\right)$ anomalies and its phenomenological implications, J. High Energy Phys. 08 (2016) 054.

[8] R. Barbieri, C. W. Murphy, and F. Senia, B-decay anomalies in a composite leptoquark model, Eur. Phys. J. C 77, 8 (2017).

[9] S. Sahoo, R. Mohanta, and A. K. Giri, Explaining the $R_{K}$ and $R_{D^{(*)}}$ anomalies with vector leptoquarks, Phys. Rev. D 95, 035027 (2017).

[10] N. Assad, B. Fornal, and B. Grinstein, Baryon number and lepton universality violation in leptoquark and diquark models, Phys. Lett. B 777, 324 (2018).

[11] D. Buttazzo, A. Greljo, G. Isidori, and D. Marzocca, B-physics anomalies: A guide to combined explanations, J. High Energy Phys. 11 (2017) 044.

[12] L. Di Luzio, A. Greljo, and M. Nardecchia, Gauge leptoquark as the origin of B-physics anomalies, Phys. Rev. D 96, 115011 (2017).

[13] D. Bečirević and O. Sumensari, A leptoquark model to accommodate $R_{K}^{\exp }<R_{K}^{\mathrm{SM}}$ and $R_{K^{*}}^{\exp }<R_{K^{*}}^{\mathrm{SM}}$, J. High Energy Phys. 08 (2017) 104.

[14] A. Angelescu, D. Bečirević, D. Faroughy, and O. Sumensari, Closing the window on single leptoquark solutions to the $B$-physics anomalies, J. High Energy Phys. 10 (2018) 183.

[15] S. Iguro, T. Kitahara, Y. Omura, R. Watanabe, and K. Yamamoto, $\mathrm{D}^{*}$ polarization vs $R_{D^{(*)}}$ anomalies in the leptoquark models, J. High Energy Phys. 02 (2019) 194.

[16] C. Cornella, J. Fuentes-Martin, and G. Isidori, Revisiting the vector leptoquark explanation of the B-physics anomalies, J. High Energy Phys. 07 (2019) 168.

[17] J. C. Pati and A. Salam, Lepton number as the fourth color, Phys. Rev. D 10, 275 (1974); Erratum, Phys. Rev. D 11, 703 (1975).

[18] Z. Poh, S. Raby, and Z.-z. Wang, Pati-Salam SUSY GUT with Yukawa unification, Phys. Rev. D 95, 115025 (2017).

[19] P. Hung, A. Buras, and J. Bjorken, Petite unification of quarks and leptons, Phys. Rev. D 25, 805 (1982).

[20] G. Valencia and S. Willenbrock, Quark-lepton unification and rare meson decays, Phys. Rev. D 50, 6843 (1994).

[21] L. Calibbi, A. Crivellin, and T. Li, Model of vector leptoquarks in view of the $B$-physics anomalies, Phys. Rev. D 98, 115002 (2018).

[22] M. J. Dolan, T. P. Dutka, and R. R. Volkas, Lowering the scale of Pati-Salam breaking through seesaw mixing, arXiv:2012.05976.

[23] M. Bordone, C. Cornella, J. Fuentes-Martin, and G. Isidori, A three-site gauge model for flavor hierarchies and flavor anomalies, Phys. Lett. B 779, 317 (2018).

[24] A. Greljo and B. A. Stefanek, Third family quark-lepton unification at the TeV scale, Phys. Lett. B 782, 131 (2018).

[25] M. Bordone, C. Cornella, J. Fuentes-Martín, and G. Isidori, Low-energy signatures of the $\mathrm{PS}^{3}$ model: From $B$-physics anomalies to LFV, J. High Energy Phys. 10 (2018) 148.

[26] L. Di Luzio, J. Fuentes-Martin, A. Greljo, M. Nardecchia, and S. Renner, Maximal flavor violation: A Cabibbo mechanism for leptoquarks, J. High Energy Phys. 11 (2018) 081.
[27] J. Fuentes-Martín, G. Isidori, M. König, and N. Selimović, Vector leptoquarks beyond tree level III: Vectorlike fermions and flavor-changing transitions, Phys. Rev. D 102, 115015 (2020).

[28] J. Fuentes-Martín and P. Stangl, Third-family quark-lepton unification with a fundamental composite Higgs, Phys. Lett. B 811, 135953 (2020).

[29] D. Guadagnoli, M. Reboud, and P. Stangl, The dark side of 4321, J. High Energy Phys. 10 (2020) 084.

[30] R. Aaij et al. (LHCb Collaboration), Measurement of Form-Factor-Independent Observables in the Decay $B^{0} \rightarrow K^{* 0} \mu^{+} \mu^{-}$, Phys. Rev. Lett. 111, 191801 (2013).

[31] R. Aaij et al. (LHCb Collaboration), Test of lepton universality with $B^{0} \rightarrow K^{* 0} \ell^{+} \ell^{-}$decays, J. High Energy Phys. 08 (2017) 055.

[32] R. Aaij et al. (LHCb Collaboration), Test of Lepton Universality Using $\mathrm{B}^{+} \rightarrow \mathrm{K}^{+} \ell^{+} \ell^{-}$Decays, Phys. Rev. Lett. 113, 151601 (2014).

[33] R. Aaij et al. (LHCb Collaboration), Search for LeptonUniversality Violation in $B^{+} \rightarrow K^{+} \ell^{+} \ell^{-}$Decays, Phys. Rev. Lett. 122, 191801 (2019).

[34] R. Aaij et al. (LHCb Collaboration), Angular analysis of the $B^{0} \rightarrow K^{* 0} \mu^{+} \mu^{-}$decay using $3 \mathrm{fb}^{-1}$ of integrated luminosity, J. High Energy Phys. 02 (2016) 104.

[35] R. Aaij et al. (LHCb Collaboration), Measurement of $C P$-Averaged Observables in the $B^{0} \rightarrow K^{* 0} \mu^{+} \mu^{-}$Decay, Phys. Rev. Lett. 125, 011802 (2020).

[36] R. Aaij et al. (LHCb Collaboration), Angular analysis of the $B^{+} \rightarrow K^{*+} \mu^{+} \mu^{-}$decay, arXiv:2012.13241.

[37] J. Lees et al. (BABAR Collaboration), Evidence for an Excess of $\bar{B} \rightarrow D^{(*)} \tau^{-} \bar{\nu}_{\tau}$ Decays, Phys. Rev. Lett. 109, 101802 (2012).

[38] J. Lees et al. (BABAR Collaboration), Measurement of an excess of $\bar{B} \rightarrow D^{(*)} \tau^{-} \bar{\nu}_{\tau}$ decays and implications for charged Higgs bosons, Phys. Rev. D 88, 072012 (2013).

[39] M. Huschle et al. (Belle Collaboration), Measurement of the branching ratio of $\bar{B} \rightarrow D^{(*)} \tau^{-} \bar{\nu}_{\tau}$ relative to $\bar{B} \rightarrow$ $D^{(*)} \ell^{-} \bar{\nu}_{\ell}$ decays with hadronic tagging at Belle, Phys. Rev. D 92, 072014 (2015).

[40] Y. Sato et al. (Belle Collaboration), Measurement of the branching ratio of $\bar{B}^{0} \rightarrow D^{*+} \tau^{-} \bar{\nu}_{\tau}$ relative to $\bar{B}^{0} \rightarrow$ $D^{*+} \ell^{-} \bar{\nu}_{\ell}$ decays with a semileptonic tagging method, Phys. Rev. D 94, 072007 (2016).

[41] S. Hirose et al. (Belle Collaboration), Measurement of the $\tau$ Lepton Polarization and $R\left(D^{*}\right)$ in the Decay $\bar{B} \rightarrow D^{*} \tau^{-} \bar{\nu}_{\tau}$, Phys. Rev. Lett. 118, 211801 (2017).

[42] A. Abdesselam et al. (Belle Collaboration), Measurement of $\mathcal{R}(D)$ and $\mathcal{R}\left(D^{*}\right)$ with a semileptonic tagging method, arXiv:1904.08794.

[43] R. Aaij et al. (LHCb Collaboration), Measurement of the Ratio of Branching Fractions $\mathcal{B}\left(\bar{B}^{0} \rightarrow D^{*+} \tau^{-} \bar{\nu}_{\tau}\right) /$ $\mathcal{B}\left(\bar{B}^{0} \rightarrow D^{*+} \mu^{-} \bar{\nu}_{\mu}\right)$, Phys. Rev. Lett. 115, 111803 (2015).

[44] R. Aaij et al. (LHCb Collaboration), Test of lepton flavor universality by the measurement of the $B^{0} \rightarrow D^{*-} \tau^{+} \nu_{\tau}$ branching fraction using three-prong $\tau$ decays, Phys. Rev. D 97, 072013 (2018).

[45] F. U. Bernlochner, Z. Ligeti, M. Papucci, and D. J. Robinson, Combined analysis of semileptonic $B$ decays to $D$ and $D^{*}$ : $R\left(D^{(*)}\right),\left|V_{c b}\right|$, and new physics, Phys. Rev. D 95, 115008 (2017); Erratum, Phys. Rev. D 97, 059902 (2018). 
[46] D. Bigi, P. Gambino, and S. Schacht, $R\left(D^{*}\right),\left|V_{c b}\right|$, and the heavy quark symmetry relations between form factors, J. High Energy Phys. 11 (2017) 061.

[47] M. Blanke and A. Crivellin, $B$ Meson Anomalies in a PatiSalam Model Within the Randall-Sundrum Background, Phys. Rev. Lett. 121, 011801 (2018).

[48] M. Bordone, M. Jung, and D. van Dyk, Theory determination of $\bar{B} \rightarrow D^{(*)} \ell^{-} \bar{\nu}$ form factors at $\mathcal{O}\left(1 / m_{c}^{2}\right)$, Eur. Phys. J. C 80, 74 (2020).

[49] Y. S. Amhis et al. (HFLAV Collaboration), Averages of $b$ hadron, $c$-hadron, and $\tau$-lepton properties as of 2018, arXiv:1909.12524.

[50] S. Iguro and R. Watanabe, Bayesian fit analysis to full distribution data of $\overline{\mathrm{B}} \rightarrow \mathrm{D}^{(*)} \ell \bar{\nu}:\left|\mathrm{V}_{\mathrm{cb}}\right|$ determination and new physics constraints, J. High Energy Phys. 08 (2020) 006.

[51] B. Bhattacharya, A. Datta, D. London, and S. Shivashankara, Simultaneous explanation of the $R_{K}$ and $R\left(D^{(*)}\right)$ puzzles, Phys. Lett. B 742, 370 (2015).

[52] R. Barbieri, G. Isidori, A. Pattori, and F. Senia, Anomalies in $B$-decays and $U(2)$ flavor symmetry, Eur. Phys. J. C 76, 67 (2016).

[53] D. Das, C. Hati, G. Kumar, and N. Mahajan, Towards a unified explanation of $R_{D^{(*)}}, R_{K}$ and $(g-2)_{\mu}$ anomalies in a left-right model with leptoquarks, Phys. Rev. D 94, 055034 (2016).

[54] U. Aydemir, T. Mandal, and S. Mitra, Addressing the $\mathbf{R}_{D^{(*)}}$ anomalies with an $\mathbf{S}_{1}$ leptoquark from $\mathbf{S O}(\mathbf{1 0})$ grand unification, Phys. Rev. D 101, 015011 (2020).

[55] A. Bhaskar, D. Das, T. Mandal, S. Mitra, and C. Neeraj, Precise limits on the charge-2/3 $U_{1}$ vector leptoquark, arXiv:2101.12069.

[56] W. Altmannshofer, S. Gori, M. Pospelov, and I. Yavin, Quark flavor transitions in $L_{\mu}-L_{\tau}$ models, Phys. Rev. D 89, 095033 (2014).

[57] A. Crivellin, G. D'Ambrosio, and J. Heeck, Explaining $h \rightarrow \mu^{ \pm} \tau^{\mp}, B \rightarrow K^{*} \mu^{+} \mu^{-}$and $B \rightarrow K \mu^{+} \mu^{-} / B \rightarrow K e^{+} e^{-}$ in a Two-Higgs-Doublet Model with Gauged $L_{\mu}-L_{\tau}$, Phys. Rev. Lett. 114, 151801 (2015).

[58] S. Raby and A. Trautner, Vectorlike chiral fourth family to explain muon anomalies, Phys. Rev. D 97, 095006 (2018).

[59] S. F. King, Flavorful $Z^{\prime}$ models for $R_{K^{(*)}}$, J. High Energy Phys. 08 (2017) 019.

[60] A. Falkowski, S. F. King, E. Perdomo, and M. Pierre, Flavorful $Z^{\prime}$ portal for vectorlike neutrino dark matter and $R_{K^{(*)}}$, J. High Energy Phys. 08 (2018) 061.

[61] J. Kawamura, S. Raby, and A. Trautner, Complete vectorlike fourth family and new $\mathrm{U}(1)$ ' for muon anomalies, Phys. Rev. D 100, 055030 (2019).

[62] J. Kawamura, S. Raby, and A. Trautner, Complete vectorlike fourth family with U(1)': A global analysis, Phys. Rev. D 101, 035026 (2020).

[63] B. Gripaios, M. Nardecchia, and S. A. Renner, Linear flavor violation and anomalies in B physics, J. High Energy Phys. 06 (2016) 083.

[64] P. Arnan, L. Hofer, F. Mescia, and A. Crivellin, Loop effects of heavy new scalars and fermions in $b \rightarrow s \mu^{+} \mu^{-}$, J. High Energy Phys. 04 (2017) 043.
[65] B. Grinstein, S. Pokorski, and G. G. Ross, Lepton nonuniversality in $B$ decays and fermion mass structure, J. High Energy Phys. 12 (2018) 079.

[66] P. Arnan, A. Crivellin, M. Fedele, and F. Mescia, Generic loop effects of new scalars and fermions in $b \rightarrow s \ell^{+} \ell^{-}$and a vectorlike 4th generation, arXiv:1904.05890.

[67] C.-W. Chiang and H. Okada, A simple model for explaining muon-related anomalies and dark matter, arXiv:1711 .07365 .

[68] J. M. Cline and J. M. Cornell, $R\left(K^{(*)}\right)$ from dark matter exchange, Phys. Lett. B 782, 232 (2018).

[69] J. Kawamura, S. Okawa, and Y. Omura, Interplay between the $b \rightarrow s \ell \ell$ anomalies and dark matter physics, Phys. Rev. D 96, 075041 (2017).

[70] B. Barman, D. Borah, L. Mukherjee, and S. Nandi, Correlating the anomalous results in $b \rightarrow s$ decays with inert Higgs doublet dark matter and muon $(g-2)$, arXiv:1808.06639.

[71] D. G. Cerdeño, A. Cheek, P. Martín-Ramiro, and J. M. Moreno, B anomalies and dark matter: A complex connection, Eur. Phys. J. C 79, 517 (2019).

[72] G. Arcadi, L. Calibbi, M. Fedele, and F. Mescia, Systematic approach to $B$-physics anomalies and $t$-channel dark matter, arXiv:2103.09835.

[73] A. Crivellin, C. Greub, and A. Kokulu, Explaining $B \rightarrow D \tau \nu, B \rightarrow D^{*} \tau \nu$ and $B \rightarrow \tau \nu$ in a $2 \mathrm{HDM}$ of type III, Phys. Rev. D 86, 054014 (2012).

[74] M. Tanaka and R. Watanabe, New physics in the weak interaction of $\bar{B} \rightarrow D^{(*)} \tau \bar{\nu}$, Phys. Rev. D 87, 034028 (2013).

[75] A. Celis, M. Jung, X.-Q. Li, and A. Pich, Sensitivity to charged scalars in $\mathbf{B} \rightarrow \mathbf{D}^{(*)} \boldsymbol{\tau} \boldsymbol{\nu}_{\boldsymbol{\tau}}$ and $\mathbf{B} \rightarrow \tau \boldsymbol{\nu}_{\boldsymbol{\tau}}$ decays, J. High Energy Phys. 01 (2013) 054.

[76] A. Crivellin, A. Kokulu, and C. Greub, Flavor-phenomenology of two-Higgs-doublet models with generic Yukawa structure, Phys. Rev. D 87, 094031 (2013).

[77] A. Crivellin, J. Heeck, and P. Stoffer, A Perturbed LeptonSpecific Two-Higgs-Doublet Model Facing Experimental Hints for Physics Beyond the Standard Model, Phys. Rev. Lett. 116, 081801 (2016).

[78] C.-H. Chen and T. Nomura, Charged-Higgs on $R_{D^{(*)}}, \tau$ polarization, and FBA, Eur. Phys. J. C 77, 631 (2017).

[79] S. Iguro and $\mathrm{K}$. Tobe, $R\left(D^{(*)}\right)$ in a general two Higgs doublet model, Nucl. Phys. B925, 560 (2017).

[80] S. Iguro and Y. Omura, Status of the semileptonic $B$ decays and muon $g-2$ in general 2HDMs with right-handed neutrinos, J. High Energy Phys. 05 (2018) 173.

[81] S.-P. Li and X.-Q. Li, Probing new physics signals with symmetry-restored Yukawa textures, Eur. Phys. J. C 80, 268 (2020).

[82] S.-P. Li, X.-Q. Li, Y.-D. Yang, and X. Zhang, $R_{D^{(*)}}, R_{K^{(*)}}$ and neutrino mass in the 2HDM-III with right-handed neutrinos, J. High Energy Phys. 09 (2018) 149.

[83] S. Iguro, Y. Omura, and M. Takeuchi, Test of the $R\left(D^{(*)}\right)$ anomaly at the LHC, Phys. Rev. D 99, 075013 (2019).

[84] R. Alonso, B. Grinstein, and J. Martin Camalich, Lifetime of $B_{c}^{-}$Constrains Explanations for Anomalies in $B \rightarrow D^{(*)} \tau \nu$, Phys. Rev. Lett. 118, 081802 (2017). 
[85] A. Akeroyd and C.-H. Chen, Constraint on the branching ratio of $B_{c} \rightarrow \tau \bar{\nu}$ from LEP1 and consequences for $R\left(D^{(*)}\right)$ anomaly, Phys. Rev. D 96, 075011 (2017).

[86] A. Celis, M. Jung, X.-Q. Li, and A. Pich, Scalar contributions to $b \rightarrow c(u) \tau \nu$ transitions, Phys. Lett. B 771, 168 (2017).

[87] M. Borsato, V. V. Gligorov, D. Guadagnoli, D. Martinez Santos, and O. Sumensari, Effective-field-theory arguments for pursuing lepton-flavor-violating $\mathrm{K}$ decays at LHCb, Phys. Rev. D 99, 055017 (2019).

[88] M. J. Baker, J. Fuentes-Martín, G. Isidori, and M. König, High- $p_{T}$ signatures in vector-leptoquark models, Eur. Phys. J. C 79, 334 (2019).

[89] M. Algueró, B. Capdevila, A. Crivellin, S. DescotesGenon, P. Masjuan, J. Matias, M. Novoa Brunet, and J. Virto, Emerging patterns of new physics with and without lepton flavor universal contributions, Eur. Phys. J. C 79, 714 (2019); 80, A511 (2020).

[90] S. Iguro, M. Takeuchi, and R. Watanabe, Testing leptoquark/EFT in $\bar{B} \rightarrow D^{(*)} l \bar{\nu}$ at the LHC, arXiv:2011.02486.

[91] S. Antusch and V. Maurer, Running quark and lepton parameters at various scales, J. High Energy Phys. 11 (2013) 115.

[92] G. Aad et al. (ATLAS Collaboration), Search for highmass dilepton resonances using $139 \mathrm{fb}^{-1}$ of $p p$ collision data collected at $\sqrt{s}=13 \mathrm{TeV}$ with the ATLAS detector, Phys. Lett. B 796, 68 (2019).

[93] M. Aaboud et al. (ATLAS Collaboration), Combination of the Searches for Pair-Produced Vectorlike Partners of the Third-Generation Quarks at $\sqrt{s}=13 \mathrm{TeV}$ with the ATLAS Detector, Phys. Rev. Lett. 121, 211801 (2018).

[94] J. Heeck and D. Teresi, Pati-Salam explanations of the B-meson anomalies, J. High Energy Phys. 12 (2018) 103.

[95] P. Zyla et al. (Particle Data Group Collaboration), Review of particle physics, Prog. Theor. Exp. Phys. 2020, 083C01 (2020).

[96] W. H. Bertl et al. (SINDRUM II Collaboration), A search for muon to electron conversion in muonic gold, Eur. Phys. J. C 47, 337 (2006).

[97] H. Natori (DeeMe Collaboration), DeeMe experimentAn experimental search for a mu-e conversion reaction at J-PARC MLF, Nucl. Phys. B, Proc. Suppl. 248-250, 52 (2014).

[98] Y. Kuno (COMET Collaboration), A search for muon-toelectron conversion at J-PARC: The COMET experiment, Prog. Theor. Exp. Phys. (2013), 022C01.

[99] R. J. Abrams et al. (Mu2e Collaboration), Mu2e conceptual design report, arXiv:1211.7019.

[100] L. Lavoura, General formulas for $f(1) \rightarrow f(2) \gamma$, Eur. Phys. J. C 29, 191 (2003).

[101] A. Baldini et al. (MEG Collaboration), Search for the lepton flavor violating decay $\mu^{+} \rightarrow \mathrm{e}^{+} \gamma$ with the full dataset of the MEG experiment, Eur. Phys. J. C 76, 434 (2016).
[102] A. M. Baldini et al. (MEG II Collaboration), The design of the MEG II experiment, Eur. Phys. J. C 78, 380 (2018).

[103] A. J. Buras, S. Jager, and J. Urban, Master formulas for Delta $F=2$ NLO QCD factors in the standard model and beyond, Nucl. Phys. B605, 600 (2001).

[104] A. J. Buras, Weak Hamiltonian, $C P$ violation and rare decays, in Probing the Standard Model of Particle Interactions. Proceedings, Summer School in Theoretical Physics, NATO Advanced Study Institute, 68th session, Les Houches, France, 1997. Pt. 1, 2 (1998), pp. 281-539.

[105] M. Bona et al. (UTfit Collaboration), The 2004 UTfit collaboration report on the status of the unitarity triangle in the standard model, J. High Energy Phys. 07 (2005) 028.

[106] M. Bona et al. (UTfit Collaboration), Model-independent constraints on $\Delta F=2$ operators and the scale of new physics, J. High Energy Phys. 03 (2008) 049.

[107] L. Di Luzio, M. Kirk, A. Lenz, and T. Rauh, $\Delta M_{s}$ theory precision confronts flavor anomalies, J. High Energy Phys. 12 (2019) 009.

[108] R. N. Mohapatra and R. E. Marshak, Local B-L Symmetry of Electroweak Interactions, Majorana Neutrinos and Neutron Oscillations, Phys. Rev. Lett. 44, 1316 (1980); Erratum, Phys. Rev. Lett. 44, 1644 (1980).

[109] D. G. Phillips, II et al., Neutron-antineutron oscillations: Theoretical status and experimental prospects, Phys. Rep. 612, 1 (2016).

[110] K. Abe et al. (Super-Kamiokande Collaboration), The search for $n-\bar{n}$ oscillation in Super-Kamiokande I, Phys. Rev. D 91, 072006 (2015).

[111] R. Aaij et al. (LHCb Collaboration), Test of lepton universality in beauty-quark decays, arXiv:2103.11769.

[112] S. Aoki et al. (Flavour Lattice Averaging Group Collaboration), FLAG review 2019: Flavour Lattice Averaging Group (FLAG), Eur. Phys. J. C 80, 113, 2020.

[113] V. Cirigliano, R. Kitano, Y. Okada, and P. Tuzon, On the model discriminating power of $\mu \rightarrow e$ conversion in nuclei, Phys. Rev. D 80, 013002 (2009).

[114] P. Junnarkar and A. Walker-Loud, Scalar strange content of the nucleon from lattice QCD, Phys. Rev. D 87, 114510 (2013).

[115] R. Kitano, M. Koike, and Y. Okada, Detailed calculation of lepton flavor violating muon electron conversion rate for various nuclei, Phys. Rev. D 66, 096002 (2002); Erratum, Phys. Rev. D 76, 059902 (2007).

[116] T. Suzuki, D. F. Measday, and J. Roalsvig, Total nuclear capture rates for negative muons, Phys. Rev. C 35, 2212 (1987).

[117] M. Blanke, A. Crivellin, S. de Boer, T. Kitahara, M. Moscati, U. Nierste, and I. Nišandžić, Impact of polarization observables and $B_{c} \rightarrow \tau \nu$ on new physics explanations of the $b \rightarrow c \tau \nu$ anomaly, Phys. Rev. D 99, 075006 (2019). 